\title{
WestVirginiaUniversity
}

THE RESEARCH REPOSITORY @ WVU

Graduate Theses, Dissertations, and Problem Reports

1996

\section{Nucleation and Growth of GaN on Sapphire by MBE}

\author{
Steven Buczkowski \\ West Virginia University
}

Follow this and additional works at: https://researchrepository.wvu.edu/etd

Part of the Chemistry Commons

\section{Recommended Citation}

Buczkowski, Steven, "Nucleation and Growth of GaN on Sapphire by MBE" (1996). Graduate Theses, Dissertations, and Problem Reports. 5017.

https://researchrepository.wvu.edu/etd/5017

This Thesis is protected by copyright and/or related rights. It has been brought to you by the The Research Repository @ WVU with permission from the rights-holder(s). You are free to use this Thesis in any way that is permitted by the copyright and related rights legislation that applies to your use. For other uses you must obtain permission from the rights-holder(s) directly, unless additional rights are indicated by a Creative Commons license in the record and/ or on the work itself. This Thesis has been accepted for inclusion in WVU Graduate Theses, Dissertations, and Problem Reports collection by an authorized administrator of The Research Repository @ WVU. For more information, please contact researchrepository@mail.wvu.edu. 


\title{
Nucleation and Growth of GaN on Sapphire by MBE
}

\author{
Thesis \\ Submitted to the Eberly College of Arts and Sciences of \\ West Virginia University \\ In Partial Fulfillment of the Requirements for \\ The Degree of Master of Science
}

by

Steven Buczkowski

December, 1996 
GaN was grown by molecular beam epitaxy in an effort to determine nucleation and growth conditions which lead to high quality, single-crystal films. Atomic force microscopy (AFM) was used to show that growth under Ga-rich conditions promotes the nucleation of films with large nucleation domains leading to a lower density of intrinsic defects related to domain boundaries. These conditions are also shown to promote a 2-D growth mode resulting in films with a high degree of nucleation domain coalescence and surface roughnesses below $2 \mathrm{~nm}$. Addition of atomic hydrogen, using a thermally-cracked source, is shown to increase the growth rate of Ga-rich growths by a factor of two. The hydrogen appears to be altering the growth kinetics to increase the residence time of nitrogen atoms at the substrate surface. The addition of hydrogen seems to have no effect on the material characteristics as probed by Hall measurements and photoluminescence. In addition, the RF source used to generate active nitrogen species was characterized by means of mass spectroscopy and optical emission spectroscopy. Under normal operating conditions for $\mathrm{GaN}$ growth, the source was shown to convert about 5\% of the total nitrogen input into neutral, nitrogen atoms and about $0.03 \%$ into nitrogen ions which may cause crystal damage during growth.
\end{abstract}


This work may have never reached this state were it not for my wife Debra's constant encouragement and ability to make sense out of MS Word. For her love and support, I am truly indebted.

I would also like to thank Dr. Myers for allowing me the opportunity to work in the lab and making this thesis possible. I've learned a great deal about myself and my abilities as a researcher through the experience and will keep this knowledge with me always.

To Dr. Giles, Dr. Lederman and Dr. Littleton; thank you for taking the time to be members of my thesis committee. Your input has been very important to the evolution of this work.

Special thanks go out to Dr. Zhonghai Yu for his help and encouragement around the lab, to Dr. Michelle Richards-Babb for teaching me how to run the AFM, and to Dr. Giles and her lab for their photoluminescence measurements. Finally, I must also say thank you to all the other people around the department who have been my friends and teachers. 


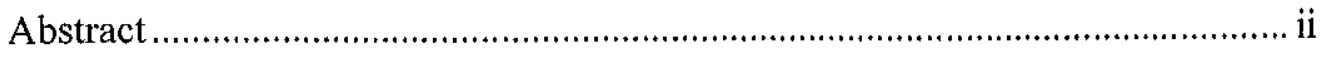

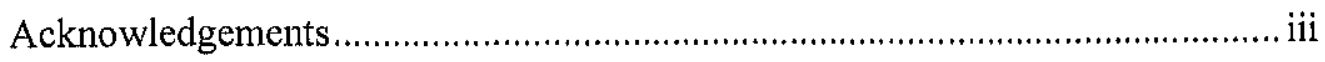

Table of Contents .................................................................................... iv

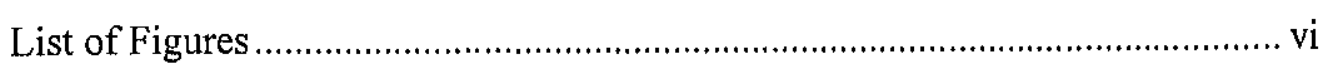

\section{Chapter}

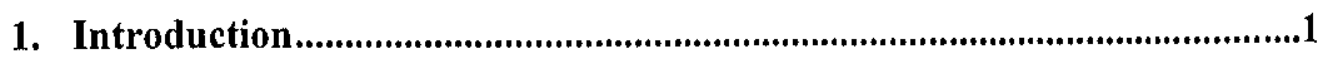

1.1 Wide Bandgap Semiconductors ...................................................

1.2 Motivation and Goals of this Research ................................................5

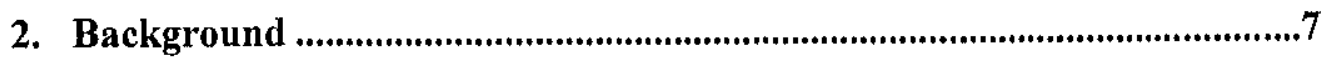

2.1 Summary of GaN Material Characteristics ........................................ 7

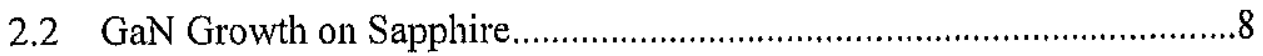

2.3 MBE Growth Kinetics ............................................................

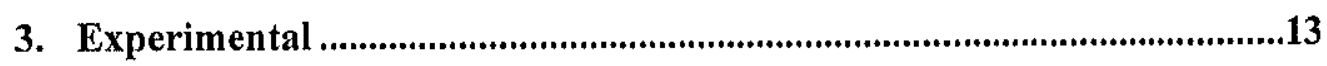

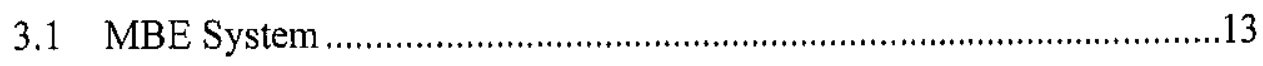

3.1.1 Chamber Description .......................................................13

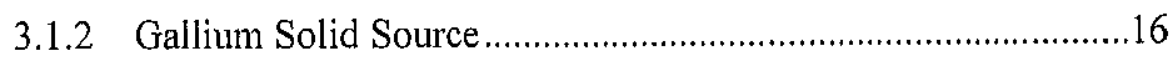

3.1.3 Atomic Hydrogen Source ..................................................19

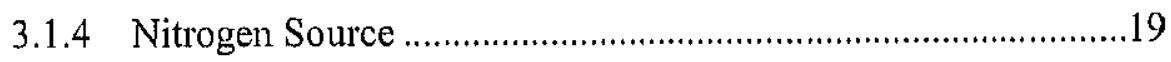

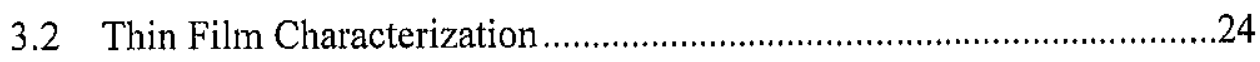

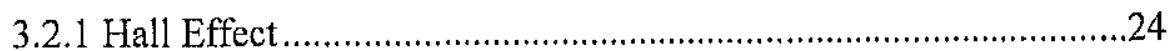

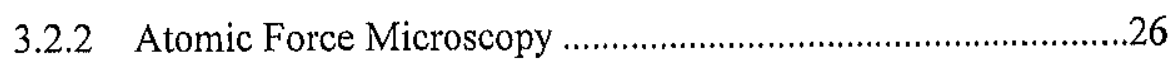

3.2.3 Photoluminescence Spectroscopy …...................................28

3.2.4 Transmission Electron Microscopy …....................................29

3.2.5 Secondary Ion Mass Spectrometry …....................................30

3.2.6 UV Fluorescence Microscopy .............................................30

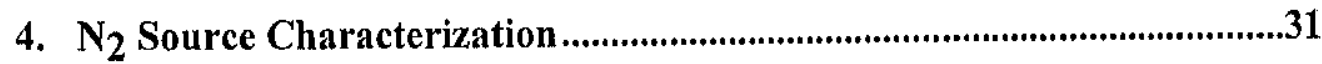

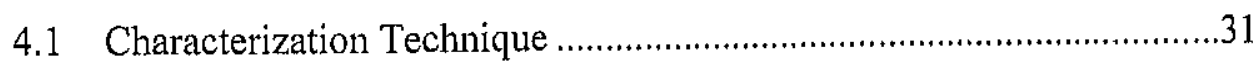


4.1.1 Mass spectrometry ............................................................

4.1.2 Optical Emission Spectroscopy ............................................37

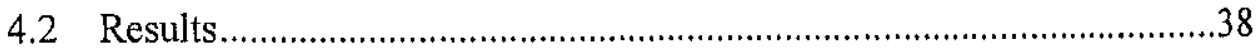

5. Nucleation and Growth of GaN .......................................................48

5.1 Nucleation and Buffer Layers .......................................................48

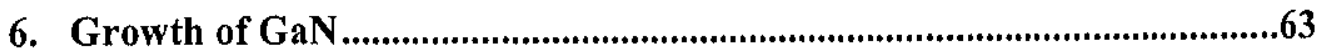

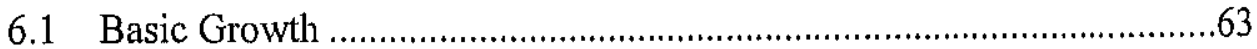

6.2 Effects of Hydrogen on the Growth of GaN .....................................73

7. Summary and Future Areas for Research...............................................78

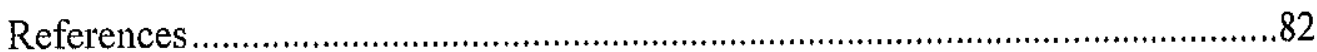

Appendix A: List of Growth Conditions for GaN Samples.................................8 
Figure 1.1 Schematic comparing lattice matches for the III-V nitrides and several common substrates. The system of interest here is the sapphire to hexagonal $\mathrm{GaN}$. The hexagonal values are for the in-plane, or ' $a$ ', spacing.

Figure 2.1 Schematic diagram of processes involved in film nucleation and growth.

Figure 3.1 Schematic diagram of an MBE system. In addition to the main chamber, source flange, load-lock and pumping system, other important components include temperature control unit, vacuum control unit and characterization unit.

Figure 3.2 EPI-40-M Modified filament knudsen effusion cell. 17

Figure 3.3 Beam equivalent pressure and equivalent atomic flux at the substrate for several Ga oven temperatures.

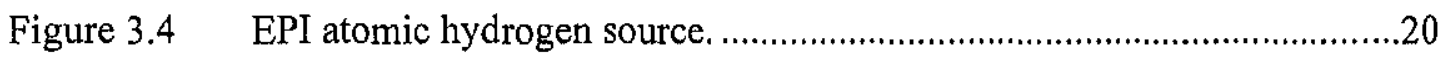

Figure 3.5 Cracking efficiency of atomic hydrogen source. ....................................21

Figure 3.6 Oxford Applied Research CARS25 Radical/Beam source for the production of atomic nitrogen .22

Figure 3.7 Schematic of a Hall measurement setup. …….........................................25

Figure 3.8 Schematic of the atomic force microscopy measurement system.

Figure 4.1 Schematic of nitrogen source characterization chamber. Source is mounted down the mass spectrometer line-of-sight. The distance from the source to the mass spec. is roughly $8 \mathrm{~cm}$.

Figure 4.2 Schematic diagram of QMS system......................................................34

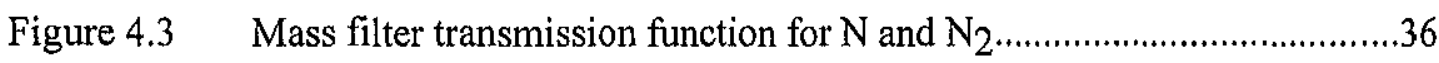

Figure 4.4 Typical spectrum of the optical emission from the CARS25 nitrogen source during operation. The labeled peaks have been related to transitions in atomic nitrogen. 
Figure 4.5 Photodiode response as a function of source pressure (Nitrogen flow for the lower plot) The upper plot is characteristic of the doping aperture, while the bottom is characteristic of the growth aperture.

Figure 4.6 Summary plot of nitrogen source mass spectra for doping aperture \#1(top). Bottom plot shows atomic nitrogen flux at substrate. Relative error in these values is estimated to be approximately $20 \%$.

Figure 4.7 Summary plot of nitrogen source mass spectra for doping aperture \#2(top). Bottom plot shows atomic nitrogen flux at substrate. Relative error in these values is estimated to be approximately $20 \%$,

Figure 4.8 Summary plot of nitrogen source mass spectra for growth aperture \#3(top). Bottom plot shows atomic nitrogen flux at substrate. Relative error in these values is estimated to be approximately $20 \%$.

Figure 4.9 Representative plots of atomic(middle) and molecular(bottom) line intensities, normalized to the $773 \mathrm{~nm}$ line intensity, from OES peakfits at fixed source pressure. Top graph is the integrated intensity from the photodiode system included with the source. Similar results were seen for all apertures studied

Figure 4.10 Representative plots of atomic(middle) and molecular(bottom) line intensities, normalized to the $773 \mathrm{~nm}$ line intensity, from OES peakfits at fixed source power. Top graph is the integrated intensity from the photodiode system included with the source. Similar results were seen for all apertures studied.

Figure 5.1 AFM micrograph of sample 9555 grown at $630^{\circ} \mathrm{C}$ substrate temperature, $2.5 \times 10^{-7}$ Torr Ga BEP, 4 SCCM N2 and 500 watts. Sapphire was exposed to nitrogen plasma prior to growth.

Figure 5.2 AFM micrograph of sample 9558 grown on a $50 \AA$ buffer layer grown at $450{ }^{\circ} \mathrm{C}$ and annealed at $630^{\circ} \mathrm{C}$. Epilayer grown at $630^{\circ} \mathrm{C}$ substrate temperature, $2.5 \times 10^{-7}$ Torr Ga BEP, 4 SCCM N2 and 500W

Figure 5.3 Representative fit of island diameter distribution to poisson distribution. Mean island sizes were extracted from similar fits.

Figure 5.4 GaN nucleation island diameter as a function of growth conditions.

Figure 5.5 Growth rate as a function of $\mathrm{Ga}$ flux for several temperatures.

Estimated error in growth rate determination is approximately $10 \%$. 
Figure 5.6 (Clockwise from top left) AFM micrographs of samples grown under $2.5 \times 10^{-7}$ Torr $\mathrm{Ga}$ at substrate temperatures of 550, 580, $600,630^{\circ} \mathrm{C}$ and $4 \mathrm{SCCM}$ nitrogen at 500 watts. Measured rms surface roughness was $>100 \mathrm{~nm}$ in all cases.

Figure 5.7 Height mode image of one of the samples shown in figure 5.6.

Figure 5.8 AFM micrographs of samples grown at $660^{\circ} \mathrm{C}$ and 4 SCCM nitrogen at 500 Watts. The top image is from a sample grown under $2.5 \times 10^{-7}$ Torr $\mathrm{Ga}$, while the lower image is from a sample grown under $5.0 \times 10^{-7}$ Torr.

Figure 5.9 AFM micrographs of samples grown at $670^{\circ} \mathrm{C}$ and 6 SCCM nitrogen at 500 Watts. The top image is from a sample grown under $5.0 \times 10^{-7}$ Torr $\mathrm{Ga}$, while the lower image is from a sample grown under $7.0 \times 10^{-7}$ Torr.

Figure 5.10 Nucleation layer grown at $660^{\circ} \mathrm{C}$ under $5.0 \times 10^{-7}$ torr Ga and 6 SCCM nitrogen at 500 watts. The mean diameter of the islands in the sample was 0.3 micron.

Figure 6.1 AFM micrographs of samples grown at $670^{\circ} \mathrm{C}$ and 6 SCCM nitrogen at 500 Watts. The top image is from a sample grown under $5.0 \times 10^{-7}$ Torr $\mathrm{Ga}$, while the lower image is from a sample grown under $7.0 \times 10^{-7}$ Torr.

Figure 6.2 AFM micrographs of samples grown at $730^{\circ} \mathrm{C}$ and 6 SCCM nitrogen at 600 Watts. The top image is from a sample grown under $5.0 \times 10^{-7}$ Torr $\mathrm{Ga}$, while the lower image is from a sample grown under $7.0 \times 10^{-7}$ Torr.

Figure 6.3 Dark field TEM image of sample 9571 (AFM image shown in figure 6.2a). 'ID' denotes an Inversion Domain. The remaining defects are non-edge type dislocations. (Photo courtesy of Dr. Linda Romano, Xerox PARC)

Figure 6.4 Schematic of Inversion Domain structure. ('S' represents a substrate step or defect which may be responsible for creating this type of defect.) (Drawing courtesy of Dr. Linda Romano, Xerox, PARC).

Figure 6.5 Photoluminescence spectra for two samples grown at $660^{\circ} \mathrm{C}$ (top) and $730^{\circ} \mathrm{C}$ (bottom). Note suppression of $2.2 \mathrm{eV}$ 'yellow' luminescence in the higher temperature growth.

Figure 6.6 Ultraviolet Fluorescence micrograph of 'yellow luminescence' seen in PL spectra. 
Figure 6.7 Graph of growth rate versus Ga flux for various growth conditions. Unfilled symbols correspond to growth conditions of similar filled symbols, but with the addition of atomic hydrogen to the growth flux.

Figure 6.8 AFM micrographs of two samples grown at $670^{\circ} \mathrm{C}$ under $5.0 \times 10^{-7}$ Torr $\mathrm{Ga}$ and $6 \mathrm{SCCM}$ nitrogen at 500 watts. Top image is from a sample grown without hydrogen in the growth flux. Bottom image is from a sample grown with $1.0 \times 10^{-6}$ Torr added to growth flux through the thermal cracker. Note smaller domain size similar to increasing nitrogen flux.

Figure 6.9 PL spectra for two samples grown with hydrogen in the growth flux. Spectra are virtually unchanged over similar non-hydrogen growths.

Figure 7.1 SIMS spectrum for sample 9567 indicating a very high level of boron inclusion. Similar results were obtained for sample 9569. This may indicate boron inclusion in all samples grown to date. 


\section{Introduction}

\subsection{Wide bandgap semiconductors}

Semiconductor technology has become increasingly important in the last fifty years, first with the advent of computer technology and more recently with the increasing drive toward optoelectronics. The need for smaller, faster and more robust electronic devices has created a tremendous push in the materials sciences to produce materials better suited to the tasks at hand/Within the last thirty years, optoelectronics have come to the forefront of technology as one solution to the size and speed demands imposed on modern electronics The III-V materials GaAs and AlGaAs have been very successful in this arend. GaAs-based high frequency switching circuits are becoming increasingly available and $\mathrm{AlGaAs}$ based laser diodes have spawned a tremendous revolution in the area of optical storage.

Currently, semiconductor optical devices routinely operate from the infra-red (IR) to green wavelengths! By developing high reliability devices which would operate into the blue and ultra-violet (UV), semiconductor based devices could emit and detect over the entire visible spectrum impacting heavily on imaging and graphics applications. For example, a blue or UV light laser diode (LD) would increase currently diffraction limited near-IR optical storage densities by more than a factor of four.

The longest wavelength of light which a semiconductor can generate or respond to is dictated, to first order, by its bandgap energy as $E_{B a n d g a p}=h c / \lambda$. The larger the bandgap, the shorter the wavelength of light that can be captured or created. In order to reach the blue end of the visual spectrum then, it is necessary to develop systems with bandgaps on the order of $2 \mathrm{ev}$ or higher. The best contenders in this field to date are the III-V nitride materials (GaN $3.4 \mathrm{eV}$; AlN $6.2 \mathrm{eV}$; InN $1.9 \mathrm{eV}$ ), $\mathrm{ZnSe}(2.67 \mathrm{eV})$ and $\mathrm{SiC}$ $(2.2 \mathrm{eV}) . \mathrm{SiC}$ has been studied very extensively and has been a major force in the blue LED market for some time. It is however limited by an indirect bandgap which reduces 
its overall light emission efficiency thereby eliminating all hope of building a $\mathrm{SiC}$ laser diode structure. $\mathrm{SiC}$ does have the advantage that it is currently one of the most promising wide bandgap materials for non-optical devices, such as bipolar and fieldeffect transistors [1]. The II-VI material ZnSe has long been considered the next step in laser diode technology but it is still very limited by problems with high contact resistance, low p-type doping and short device lifetimes related to defects in the crystal structure [2].

The III-V nitrides may prove to be the best of all possible choices. GaN, InN and AIN form a continuous alloy system with bandgaps ranging from $1.9 \mathrm{eV}$ to $6.2 \mathrm{eV}$. This would allow potential device operation ranging from red wavelengths to the far UV. The III-V nitrides are expected to have superior radiation hardness which, combined with a resistance to both high temperatures and most acids, makes them very attractive for use in space-borne electronics and harsh environments.

Considerable work has been done recently toward realizing the potential of these materials. However, even with the rapid advancement in material quality and the exhibition of numerous practical devices, many issues have yet to be resolved fully. Most groups have focused on molecular beam epitaxy (MBE), chemical vapor deposition (CVD) or their variants as the growth system of choice [3]. While metal organic CVD (MOCVD) offers significantly higher growth rates, making it attractive for commercial device applications, the presence of the large amounts of hydrogen typical of MOCVD has been shown to heavily compensate p-type dopants $[4,5]$. This has been overcome somewhat by the finding that dopant passivation can be partially reversed by either low energy (10-15 keV) electron beam irradiation or a thermal anneal in a $\mathrm{N}_{2}$ atmosphere $[6,7]$. These findings have recently led to the commercial availability of blue LEDs, based on GaN, by Nichia Chemical Industries in Japan [8] and CREE Research, Inc. in the United States. $\mathrm{MBE}$, on the other hand, offers an environment free of hydrogen for p-type material growth with no need for post-growth treatment. However, it is hindered by 
significantly lower growth rates and materials properties which are generally inferior to MOCVD grown material.

CVD related growth typically uses ammonia to provide a flux of atomic nitrogen. At the higher substrate temperatures $\left(1050^{\circ} \mathrm{C}\right)$ involved, the ammonia molecule is readily decomposed to provide nitrogen at the growth surface. In order to maintain the relatively hydrogen free $\mathrm{MBE}$ environment, most $\mathrm{MBE}$ researchers have turned to using nitrogen gas. Due to the high bond strength $(9.5 \mathrm{eV})$ of the N-N bond, thermal dissociation at the substrate, alone, produces an intolerably low flux of active nitrogen. To overcome this, MBE growth of $\mathrm{GaN}$ based on molecular nitrogen requires an auxiliary method for breaking the N-N bond. MBE researchers have split among four principle methods for overcoming this energy barrier. Most have turned to one of two types of plasma sources, either radio frequency $(\mathrm{RF})$ or electron cyclotron resonance (ECR), for this bond breaking. Others have utilized either ion sources or ammonia to provide reactive nitrogen. For the plasma and ion sources, questions still remain as to the identity of the species responsible for film growth. Both neutral nitrogen atoms and low energy ions have been shown to be beneficial while high energy ions have been shown to have deleterious effects on sample quality[9,10]. Ammonia has been used successfully in a technique commonly referred to as gas source (GS)MBE to combine aspects of MBE and MOCVD. Researchers using this technique have demonstrated MOCVD-like growth rates but questions still remain as to the effects of introducing a large hydrogen flux into the growth[11].

All Group III-nitride growths, regardless of process, share two common problems. Large single crystal substrates have yet to appear for the III-nitrides so all growth must be heteroepitaxial, and samples grown without intentional dopants still exhibit a high degree of n-type conductivity.

Heteroepitaxial growth itself is not a fundamental problem However, for $\mathrm{GaN}$ in particular, no sufficiently lattice matched substrate has been found to date (Figure 1.1). 


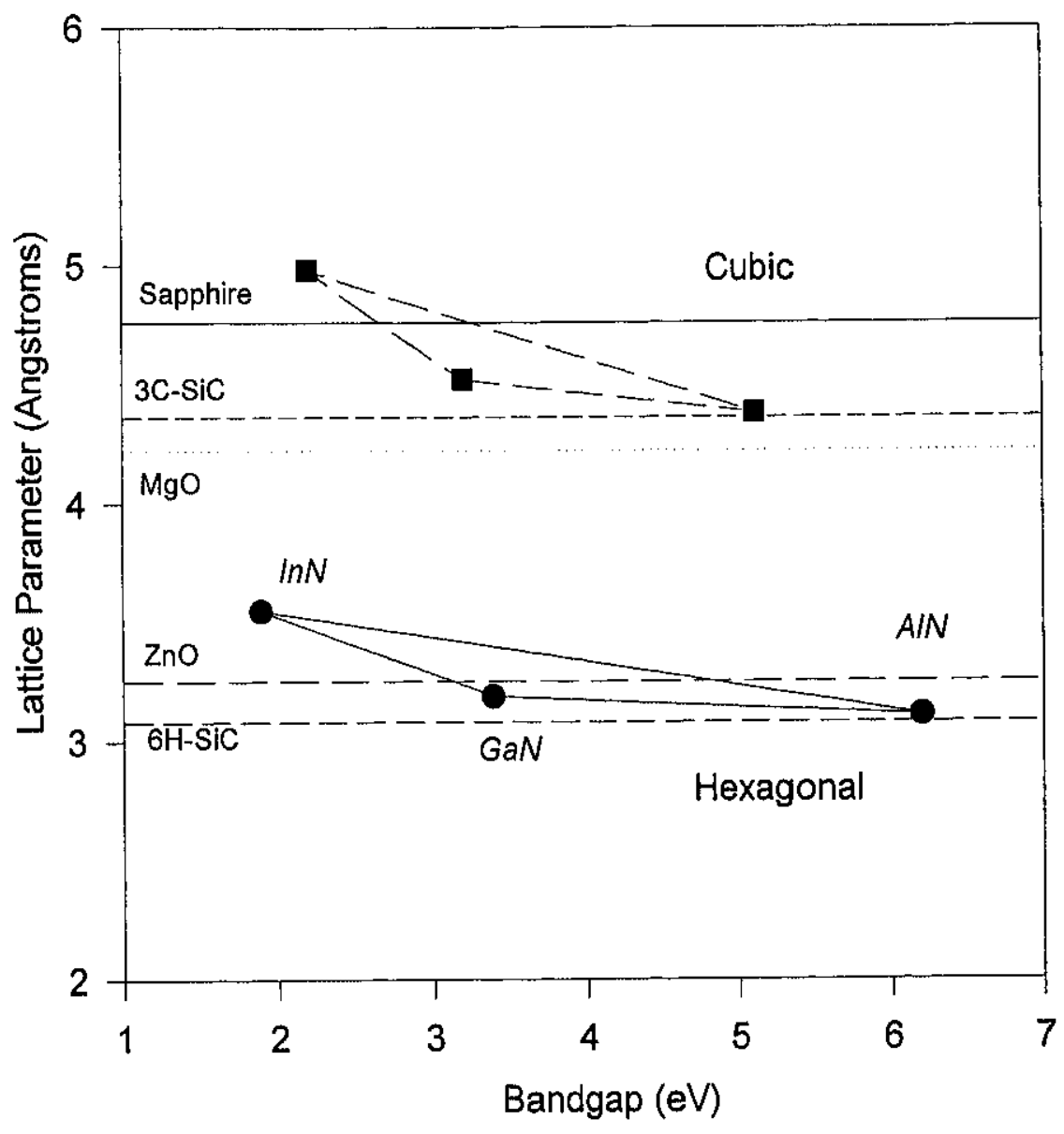

Figure 1.1 Schematic comparing lattice matches for the III-V nitrides and several common substrates. The system of interest here is the sapphire to hexagonal GaN. The hexagonal values are for the in-plane, or ' $a$ ', spacing. 
Attempts have been made to grow $\mathrm{GaN}$ on sapphire $\left(\alpha-\mathrm{Al}_{2} \mathrm{O}_{3}\right), \mathrm{ZnO}, \mathrm{MgO}, \mathrm{SiC}, \mathrm{GaAs}$, and spinel $\left(\mathrm{MgA}_{2} \mathrm{O}_{4}\right)$, among others. Even though it is one of the least lattice matched substrates available, sapphire is the most common substrate for $\mathrm{GaN}$ growth due to its availability and the convenience of pre-growth handling. Sapphire's popularity is also attributable to the successful use of buffer layers grown between the substrate and the epilayer to improve the morphology and electrical character of films grown in severely mis-matched systems In spite of the lattice mismatch, working LED structures are now commonly grown on sapphire substrates.) Due to their better lattice match, $\mathrm{ZnO}, \mathrm{MgO}$, and $\mathrm{SiC}$ are expected to become more popular as they become more readily available.

GaN, in particular, has a large problem with unintentional n-type auto doping. Undoped samples typically exhibit carrier concentrations in the $10^{17}$ to $10^{18} \mathrm{~cm}^{-3}$ range. This has been attributed to either nitrogen vacancies or oxygen impurities[12] With the recent improvements in growth practices, films are now commonly grown with carrier concentrations as low as $5 \times 10^{16} \mathrm{~cm}^{-3}$ and mobilities up to $1000 \mathrm{~cm}^{2} / \mathrm{Vs}$ at room temperature[13].

Finally, the chemical resistance of the wide bandgap materials has made it difficult to etch device structures in the grown material. This however has been mostly overcome by the use of plasma/ion beam assisted etching. A comprehensive discussion of wide-bandgap semiconductors can be found in the review articles by Strite and Morkoç[1,14].

\subsection{Motivation and Goals of this Research}

The current research program in $\mathrm{GaN}$ at WVU is directed at understanding the processes involved in the growth of $\mathrm{GaN}$, and includes investigating how these processes may be affected by the addition of non-growth species, such as hydrogen, or non-thermal energy such as irradiation with above bandgap light or high energy electrons. The work reported in this thesis represents the end-product of an effort to establish a baseline 
understanding of GaN growth and, as such, has defined directions for more detailed investigations.

The specific goals of this research project were:

1. Identify the various active nitrogen species produced by a RF nitrogen source for a variety of source operating parameters, such as, pressure, gas flow rate and plasma power.

2. Develop an understanding of the buffer layer nucleation, particularly to control the initial domain size and crystal polarity.

3. Establish a baseline for $\mathrm{GaN}$ growth, with particular emphasis on determining conditions which promote two-dimensional growth modes.

4. Perform a preliminary investigation of the effect of hydrogen on $\mathrm{GaN}$ growth kinetics through use of a thermal cracker to inject a flux of atomic hydrogen during growth. 


\section{Background}

\subsection{Summary of GaN material characteristics}

The following table lists some of the accepted values for the material characteristics of $\mathrm{GaN}$ in its two most common polytypes. The wurtzite phase is the most common of the tetrahedrally-bonded polytypes seen in $\mathrm{GaN}$ and results from growth on a hexagonal substrate. The zincblende polytype is the next most common, resulting from growth on a cubic substrate, and appears to have a slightly higher energy of formation.

\section{Fundamental properties of GaN}

\section{Wurtzite Polytype: Zincblende Polytype:}

Band-gap Energy

$\mathrm{Eg}(300 \mathrm{~K})=3.39 \mathrm{eV} \quad \mathrm{E}_{\mathrm{g}}(300 \mathrm{~K})=3.2 \cdot 3.3 \mathrm{eV}$

$\mathrm{E}_{\mathrm{g}}(1.6 \mathrm{~K})=3.50 \mathrm{eV}$

Lattice constants

$\mathrm{a}=3.189 \AA$

$\mathrm{a}=4.52 \AA$

$c=5.185 \AA$

Coefficients of

$\Delta \mathrm{a} / \mathrm{a}=5.59 \times 10^{-6} \mathrm{~K}$

Thermal Expansion

$$
\Delta \mathrm{c} / \mathrm{c}=3.17 \times 10^{-6} \mathrm{~K}
$$

Thermal conductivity

$\kappa=1.3 \mathrm{~W} / \mathrm{cm} \mathrm{K}$

Index of refraction

$\mathrm{n}(1 \mathrm{eV})=2.33$

$\mathrm{n}(3 \mathrm{eV})=2.9$

$\mathrm{n}(3.38 \mathrm{eV})=2.67$

Dielectric constant $\quad \varepsilon_{0}=9$

$\varepsilon_{\infty}=5.35$

Melting Point

Sublimes in vacuum at temperatures $>800-850^{\circ} \mathrm{C}$ 


\subsection{GaN growth on Sapphire}

Since GaN single crystal substrates do not exist at this time, growth must be performed heteroepitaxially using substrates based on other material systems. For GaN this has included sapphire $\left(\alpha-\mathrm{Al}_{2} \mathrm{O}_{3}\right), \mathrm{ZnO}, \mathrm{MgO}, \mathrm{Si}, \mathrm{SiC}, \mathrm{GaAs}$, Spinel $\left(\mathrm{MgA}_{2} \mathrm{O}_{4}\right)$ and others. Even though $\mathrm{ZnO}, \mathrm{MgO}$, and $\mathrm{SiC}$ are more closely lattice-matched to $\mathrm{GaN}$, sapphire has been the substrate of choice for many research groups because of its availability and ease of preparation prior to growth. Growth on sapphire has been attempted on a wide variety of crystal orientations with the (0001), (1012) and (1010) orientations showing the best growth relationships with GaN [15].

Despite its popularity, sapphire presents a major difficulty to the growth of $\mathrm{GaN}$. Its large lattice mismatch, $\delta=\left[\left(a_{\text {GaN }}-a_{\text {Sapphire }}\right) / a_{\text {Supphire }}\right]$, of $33 \%$ favors the formation of small, isolated three-dimensional growth islands which do not easily coalesce into largescale single crystal growth. The boundaries of the islands typically form edge-type dislocations which are difficult to eliminate in subsequent growth. These defects then affect the electrical and optical characteristics of the material by acting as traps or scatterers. One of the main thrusts of GaN work in general, and this work in particular, has been to optimize the growth conditions for $\mathrm{GaN}$ on sapphire to overcome these mismatch-induced dislocations. This issue can be approached on two fronts by finding growth conditions favorable to the elimination of existing dislocations (2-D growth), and also by promoting nucleation of larger islands so that there are fewer domain edges to generate dislocations from the start. The present work focuses more on the latter of these fronts with an ultimate goal being conditions which promote both.

The most common method for relieving the mismatch before $\mathrm{GaN}$ growth has been to grow a buffer layer with an intermediate material. Originally, most groups used a thin $(200-400 \AA)$ AlN layer grown at a low temperature $\left(500^{\circ} \mathrm{C}\right)$ and subsequent $\mathrm{GaN}$ 
growth at a higher temperature $\left(800-1000^{\circ} \mathrm{C}\right)$. The low temperature AlN layer was shown to be a uniform, amorphous layer on the sapphire which then re-crystallizes during the high temperature $\mathrm{GaN}$ growth. This approach seems to accommodate the strain between the three material layers. This same technique has since been tried with a GaN buffer layer with equal success. Kuznia et al.,[16] performed MOCVD growths to determine which material made the better buffer layer for sapphire. Their findings, by electron and $\mathrm{x}$-ray diffraction, indicate that both AIN and $\mathrm{GaN}$ are equally suited and either is significantly better than no buffer layer at all. X-ray data showed a factor of three improvement in rocking curve FWHM with the buffer layers. They also discovered that the buffer layers had an optimal thickness which promoted the highest crystallinity and the best electrical characteristics in the actual epilayer.

In addition to buffer layers, most groups expose the sapphire to a flux of active nitrogen prior to growth. The belief is that this surface nitridization produces a layer of AIN which becomes a part of the buffer layer system.

\subsection{MBE Growth Kinetics}

Before discussing how changing growth parameters affects film growth, some groundwork needs to be laid in the process of film growth itself. One of the goals in semiconductor growth is the growth of atomically smooth, single-crystal epitaxial thin films. This means that the grown film is crystallographically related to the substrate, with all of the film sharing this relationship. One of the benefits of such growth is that nearly atomically smooth surfaces allow the sharpest intermaterial junctions making such things as quantum well structures possible. Electrically and optically, a single crystal material offers the least resistance to the passage of charge carriers and photons.

Molecular beam epitaxy is an ultra-high vacuum (UHV) crystal growth technique which has become popular in the last twenty years. MBE derives its name from the fact 
that growth occurs with material fluxes low enough that chemical interactions in the gas phase are negligible. This is in contrast to chemical vapor deposition growth, where gas phase chemistry plays a major role in the deposition process. The UHV environment also considerably reduces background contamination levels.

Figure 2.1 shows schematically the competing processes involved in the epitaxial growth of a material. Starting with an incident flux of molecules coming from an overpressure of a gas or a molecular beam from a knudsen source, some fraction of the flux adsorbs onto the substrate. Once on the substrate, adsorbed molecules can diffuse across the substrate surface until they either bond with another entity or desorb from the surface. Bonding can occur to other absorbed molecules, which may also be diffusing across the surface, or to either a step edge formed by crystalline defects, a natural terrace in the substrate or the boundary of a nucleating cluster of material. In this kineticallydriven growth, one or more of the rates associated with these processes can become the limiting factor in the rate of growth.

Once nucleation begins, crystal growth follows one of three general paths: FrankVan der Merwe (layer-by-layer) growth, Stranski-Krastanov (layer-plus-island) growth, and Volmer-Weber (island) growth. In the island growth mode, the impinging atoms are more strongly bound to one another than they are to the substrate. As a result, small clusters nucleate on the substrate and proceed to grow three-dimensionally, thus retaining their separate character. The layer-by-layer growth mode is exactly opposite to the island growth. Atoms are more strongly bound to the substrate, so nucleation occurs a monolayer at a time. As further layers are added, they become slightly less energetically bound than the previous layer. If the decrease in binding energy between layers decreases monotonically toward the bulk crystal value, then the layer growth continues. Intermediate to these is the layer-plus-island growth, where the initial layer-by-layer growth is disturbed through strain or dislocation formation, and three-dimensional growth ensues. 


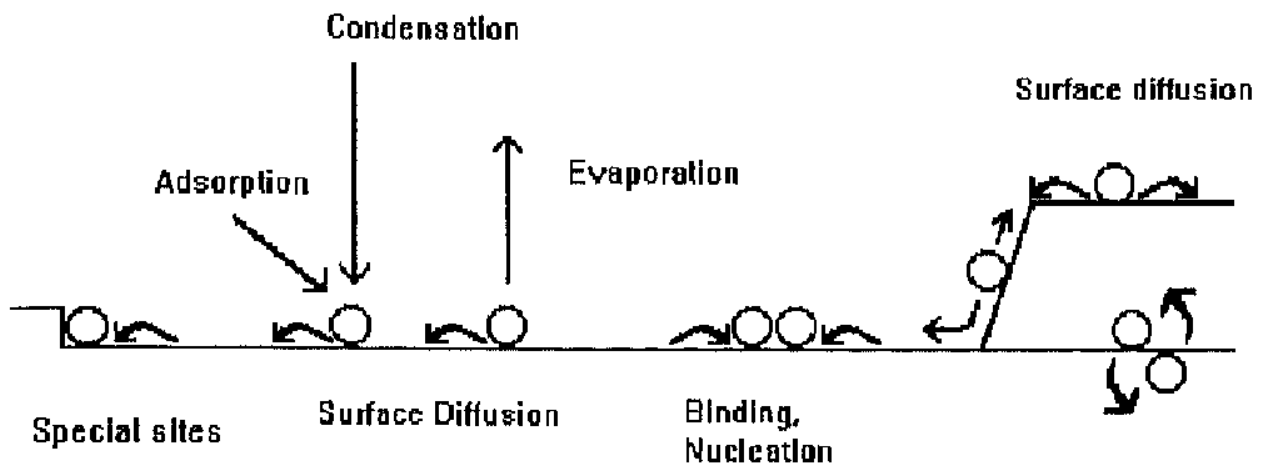

Interdlfusion

Figure 2.1 Schematic diagram of processes involved in film nucleation and growth. 
In general, the 3D growth modes produce inferior materials because the intercrystal boundaries act as traps or scatterers for charge and photons. The 2D layer growth mode is favorable since it produces the smoothest film interfaces. Also, as defects form they are pushed transversely to the growth axis by the crystal growth. By propagating 'sideways', the defects will eventually encounter either the edge of the crystal or another defect, with which it can form a closed loop defect. Either of these events terminates the defect's propagation in the crystal.

In theory, homoepitaxial growth on a clean surface should always proceed by 2-D layer growth. In practice, this is very nearly achievable. Heteroepitaxy, on the other hand, generally proceeds by some form of island growth due to lattice-mismatch or thermally induced strain. It is possible, however, to coerce a more 2-D growth pattern by careful management of growth parameters.

A more in-depth description of these processes can be found in review articles by Cho and Arthur [17], Venables, et al. [18], and Joyce [19]. 


\section{Experimental}

\subsection{MBE System}

\subsubsection{Chamber Description}

The Molecular Beam Epitaxy system used for the growths described in this work was designed and built in the West Virginia University Physics Department. Housed in a nominally Class- 1000 cleanroom facility, the ultrahigh vacuum (UHV) system consists of two chambers which can be isolated from one another by a gate valve (Figure 3.1). The smaller of the chambers functions as a load-lock which can be rapidly cycled between atmospheric pressure and $\sim 10^{-7}$ Torr to allow sample introduction into the growth chamber with minimal disturbance to its UHV environment. The load-lock consists primarily of a stainless steel six-way cross and bellows. A four-way cross and tee attached to the top of the load-lock provide space for an ion gauge to monitor the loadlock pressure and for preliminary bake-out of source ovens before they are put into the growth chamber. A magnetically coupled linear motion feedthrough with a spring-loaded bayonet mount is used to transfer sample blocks to and from the UHV chamber. Evacuation of the load-lock is accomplished with a Varian Turbo-V250 $250 \mathrm{l} / \mathrm{s}$ turbomolecular pump (TMP) which provides pumpdown times to $10^{-7}$ Torr on the order of 30 minutes. This pump also provides preliminary roughing for the UHV chamber on those occasions that it is brought up to atmospheric pressure.

The UHV growth chamber consists of a stainless steel bell jar nominally $57 \mathrm{~cm}$ high and $35.5 \mathrm{~cm}$ in di meter. The centerline of the chamber is inclined $30^{\circ}$ from the horizontal so that the sources all face upward toward the substrate. The UHV pumping system attaches to a vacuum flange which is located below the chamber. The load-lock attaches to a port on the side of the chamber. Opposite the load-lock is a liquid nitrogen $\left(\mathrm{LN}_{2}\right)$ filled cryoshroud which surrounds the substrate in the growth position. The sample 


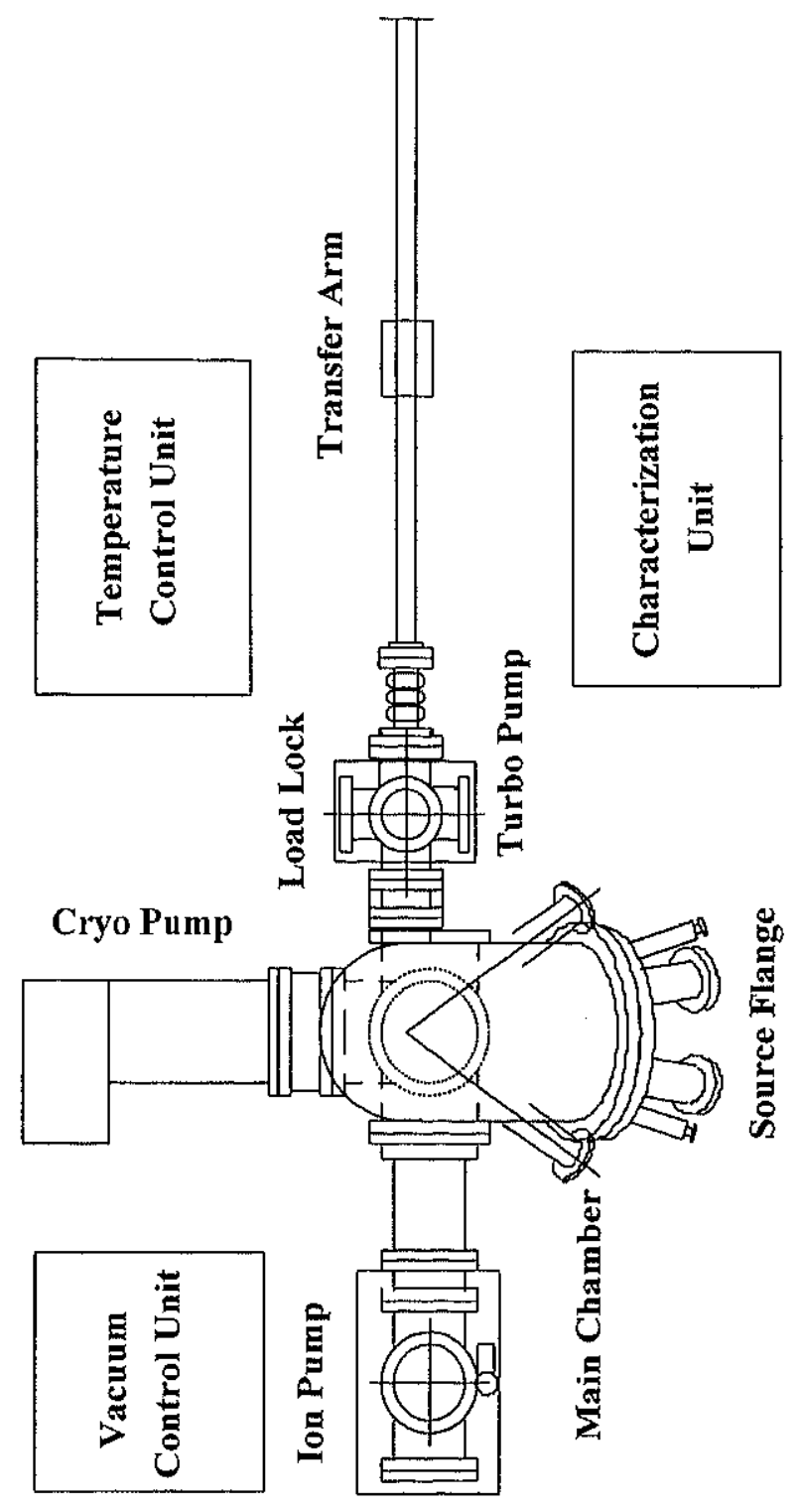

Figure 3.1 Schematic diagram of an MBE system. In addition to the main chamber, source flange, load-lock and pumping system, other important components include temperature control unit, vacuum control unit and characterization unit. 
is held in the UHV chamber on an X-Y-Z manipulation stage to which is mounted the substrate heater and a bayonet mount for the sample block.

The UHV pumping system consists of a CTI- 8 cryogenic (cryo) pump, a Varian $400 \mathrm{l} / \mathrm{s}$ ion pump and a titanium sublimation pump (TSP). The cryo-pump operates on the principle of Joule-Thompson expansion using He gas as a refrigerant. The expanding $\mathrm{He}$ gas cools a cold head to which is attached a baffle array and a high surface area absorber. The baffle array is cooled to $80 \mathrm{~K}$ and pumps by condensing species out of the vacuum space. The absorber is cooled to $15 \mathrm{~K}$ and pumps by either condensation or cryoabsorption depending on the species. The cryo-pump is a storage pump and as such loses pumping efficiency as the available pump area diminishes. To regenerate the pumping ability, the pump must be warmed and vented regularly. The ion pump ionizes incoming gas particles and then pumps by embedding the particle into a titanium (Ti) cathode or by reaction between the ion and Ti sputtered from the cathode by impinging particles. In the TSP, a thin layer of $\mathrm{Ti}$ is deposited on the walls of a $\mathrm{LN}_{2}$ reservoir. This fresh layer of $\mathrm{Ti}$ is highly reactive with gas species such as hydrogen, nitrogen and oxygen and bonds them chemically to the reservoir surface thus removing them from the vacuum space.

The source ovens enter the chamber through a source flange at the bottom of t $\mathrm{e}$ bell jar such that each oven has a nominal $29.3 \mathrm{~cm}$ source to substrate distance. Each oven has a molybdenum shutter which is used to block the flux from the oven until it is needed. The ovens are surrounded by a two layer cooling shroud which is kept at $\sim 10^{\circ} \mathrm{C}$ by an $\mathrm{H}_{2} \mathrm{O}$ : Ethylene Glycol (1:1) mixture cooled and recirculated by a Neslab (HX-200) water cooler. This shroud serves to isolate the ovens both to eliminate material cross contamination and thermal crosstalk. Oven temperatures can be controlled to better than $0.5^{\circ} \mathrm{C}$ using a Eurotherm microprocessor based controller. The nitrogen and hydrogen gas sources enter the chamber from side flanges located near the source flange. The 
sources are mounted such that the hydrogen source is $34.8 \mathrm{~cm}$ from the substrate and the nitrogen source is $35.0 \mathrm{~cm}$.

Chamber pressure is monitored using a Bayard-Alpert type ionization gauge mounted slightly behind the substrate position. The beam equivalent pressure (BEP) of the molecular flux impinging on the substrate can be measured by a similar ionization gauge mounted on a linear motion feedthrough. The linear motion allows the monitor to be positioned in front of the substrate for measurement and then retracted for growth. Residual gas composition in the chamber is monitored by a UTI $100 \mathrm{C} 1-400$ a.m.u. quadrupole mass spectrometer.

\subsubsection{Gallium Solid Source}

Molecular beams of non-gaseous species are generated in standard MBE compatible Knudsen cells. These sources all contain a resistive heater assembly, a crucible to contain the source material and a thermocouple to monitor the source temperature (Figure 3.2). The thermocouple is monitored as part of a feedback loop with a Eurotherm 818 controller which allows temperature stability to better than $0.5^{\circ} \mathrm{C}$. By precisely controlling the temperature of the source, one controls the vapor pressure of the source material in the cell and thus controls the material flux at the substrate. Figure 3.3 shows the output fluxes for this source for several operating temperatures.

The WVU MBE system uses an EPI-40-M modified filament cell to produce a flux of $\mathrm{Ga}$ atoms. This is a standard single filament source which provides additional heating in the area of the cell orifice. This so-called "hot-lip" is to prevent Ga droplet condensation at the edge of the crucible which could block the exiting flux or fall back into the molten Ga causing material spitting. 


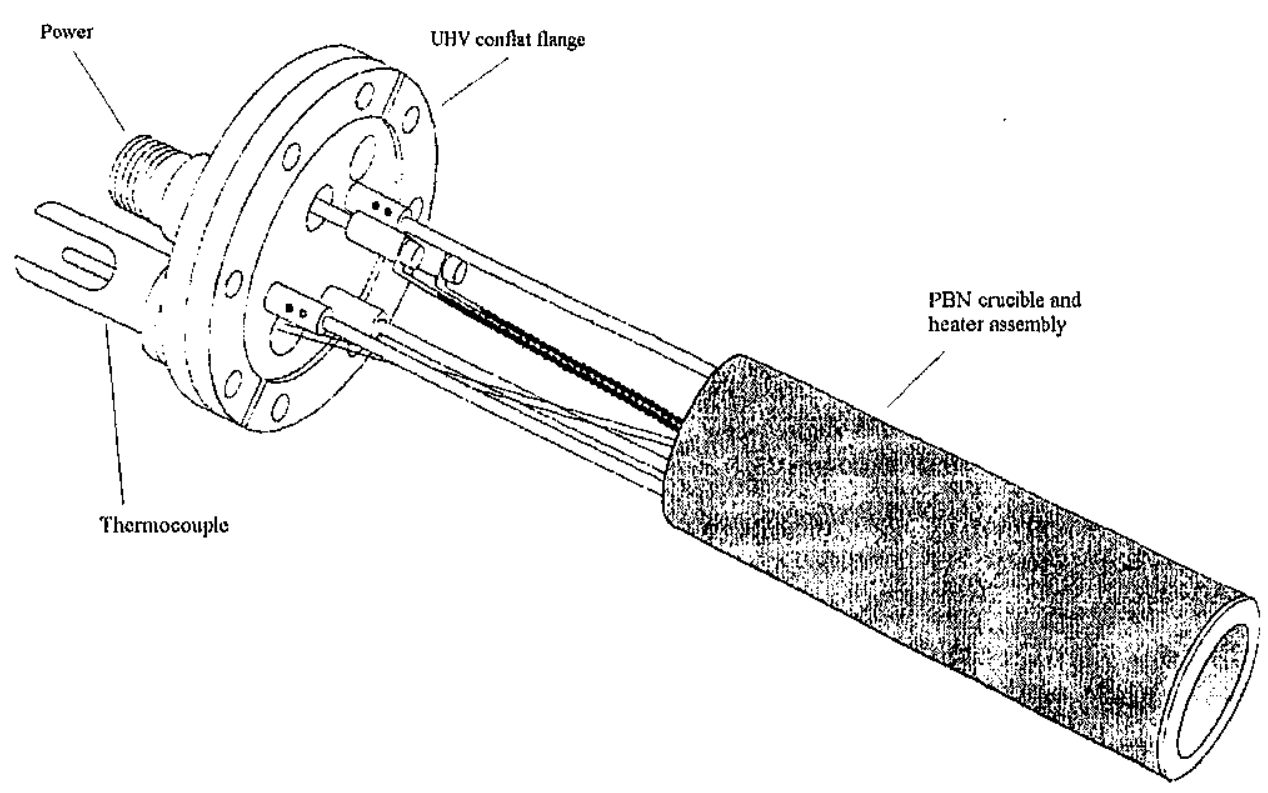

Figure 3.2 EPI-40-M Modified filament knudsen effusion cell. 


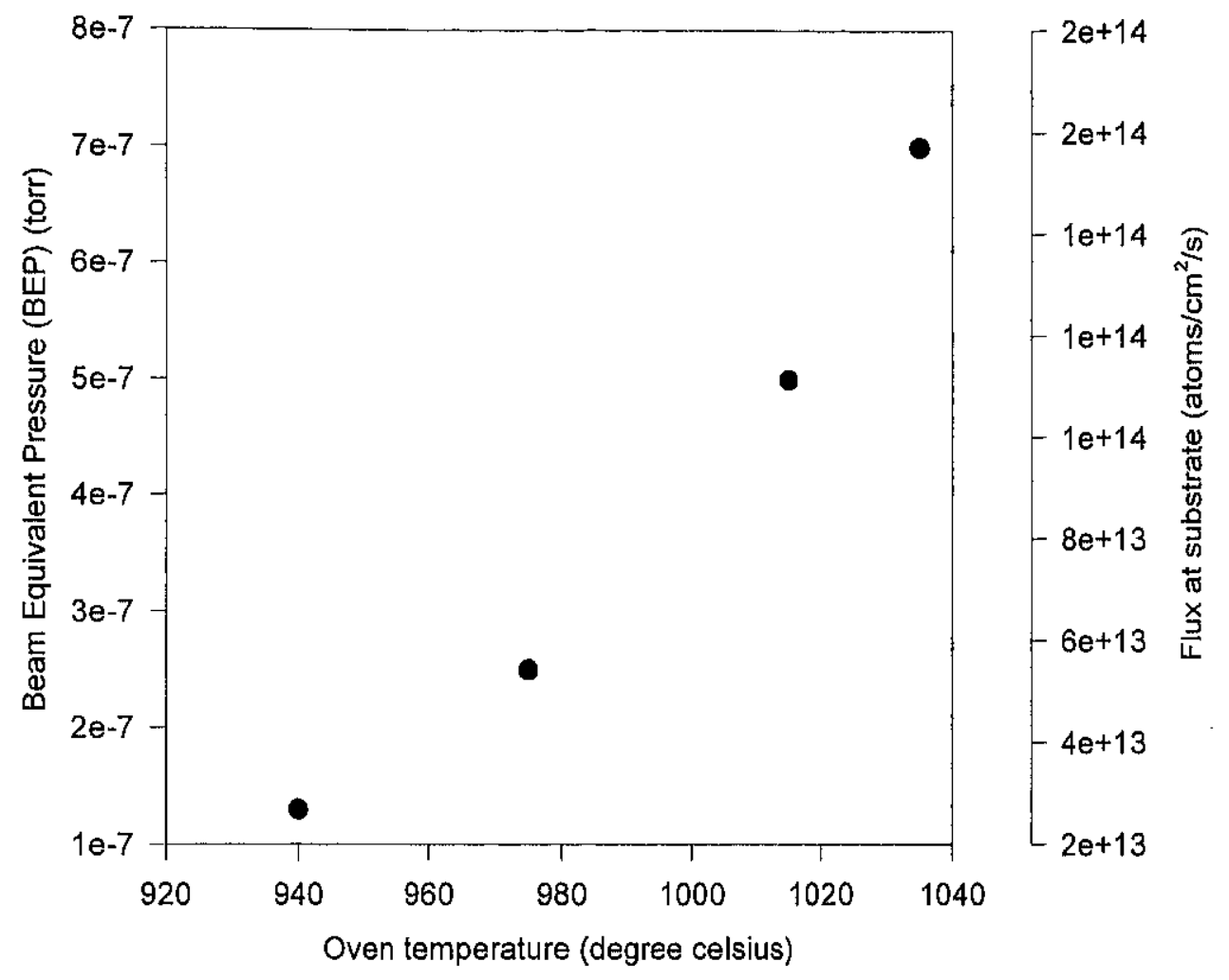

Figure 3.3 Beam equivalent pressure and equivalent atomic flux at the substrate for several Ga oven temperatures. 


\subsubsection{Atomic Hydrogen Source}

The WVU MBE chamber is equipped with an EPI-AHS-L atomic hydrogen source. The EPI hydrogen source generates atomic hydrogen by thermally cracking molecular hydrogen on a heated tungsten filament. The filament is mounted axially down a tantalum tube inside the vacuum chamber. High purity hydrogen gas flows through a UHV leak-valve into the source and across the filament (Figure 3.4). The system is also equipped with an integral water cooling jacket to prevent excessive heating of the chamber. High purity deuterium gas can also be flowed into the source through the process gas manifold.

The source is operated by passing up to 10 amps through the filament in the presence of hydrogen gas. Typical operation in the course of this study applied 9.5 amps which corresponds to a power of 430 watts. This power produces a filament temperature of about $2200^{\circ} \mathrm{C}$. (Figure 3.5 shows cracking efficiency vs. temperature) This corresponds to roughly $5 \%$ cracking efficiency with a $1 \times 10^{-6}$ Torr beam equivalent pressure, and produces a flux of $8.6 \times 10^{14} \mathrm{~s}^{-1} \mathrm{~cm}^{-2}$ hydrogen atoms at the substrate.

\subsubsection{Nitrogen Source}

Due to the large $(9.5 \mathrm{eV})$ binding energy of the nitrogen molecule, lowtemperature MBE growth of $\mathrm{GaN}$ cannot depend on the thermal dissociation of $\mathrm{N}_{2}$ at the substrate. The chamber is therefore equipped with a radio frequency $(\mathrm{RF})$ atom/radical beam source supplied by Oxford Applied Research, shown schematically in Figure 3.6, to provide active nitrogen. MBE growth using RF sources, like the one described here, and electron cyclotron resonance (ECR) sources is commonly referred to as plasma assisted (PA)MBE. Both types of sources have been successful in producing active nitrogen species with which GaN can be grown, however, ECR sources are known to generate a larger flux of nitrogen ions with energies high enough to damage the growing crystal and generate defects $[10]$. 


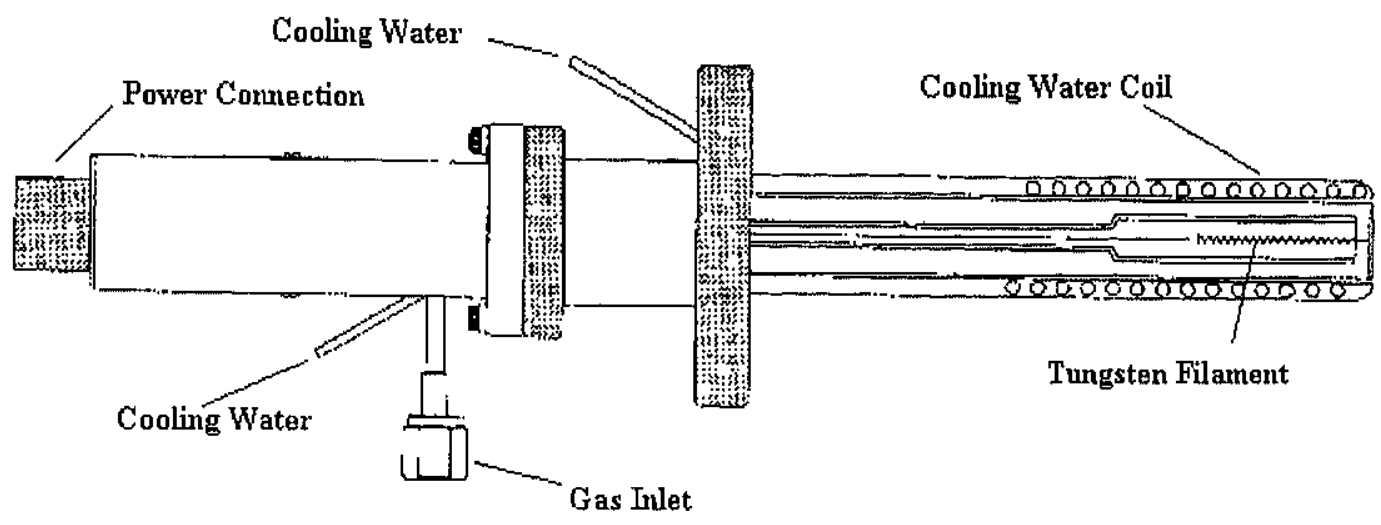

Figure 3.4 EPI atomic hydrogen source. 


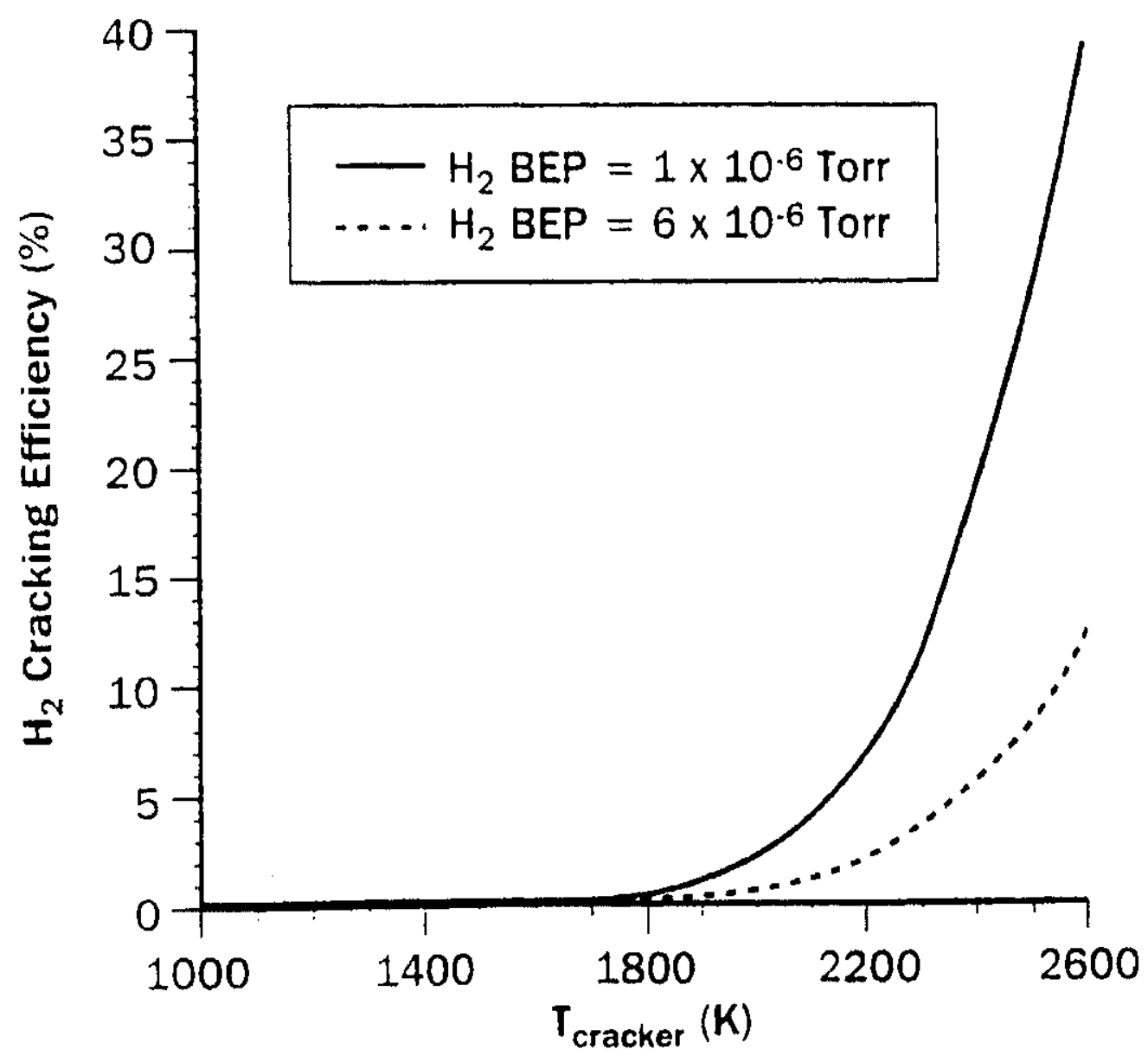

Figure 3.5 Cracking efficiency of atomic hydrogen source. 


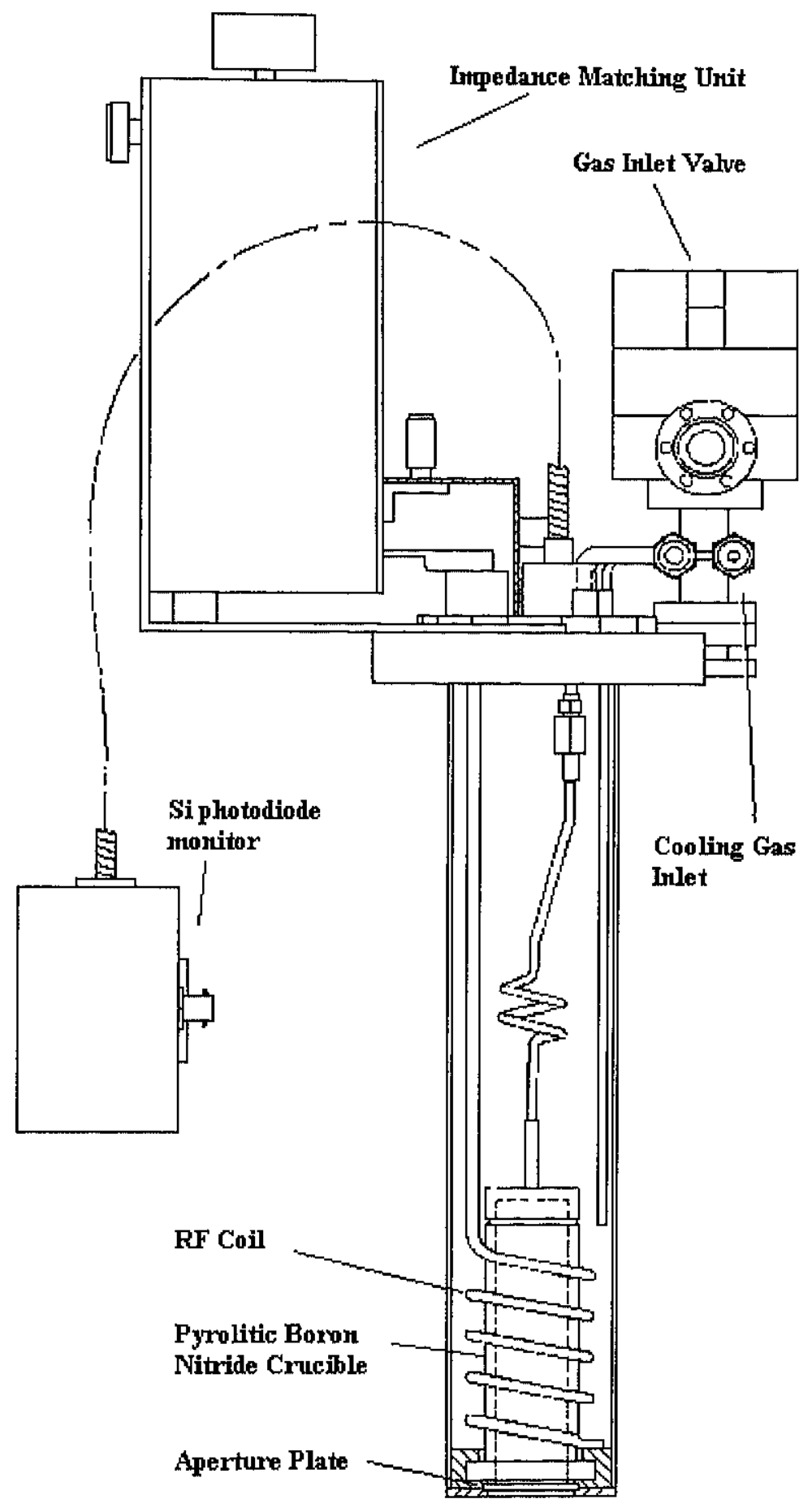

Figure 3.6 Oxford Applied Research CARS25 Radical/Beam source for the production of atomic nitrogen. 
The Oxford CARS25 source is designed to fit into a standard MBE furnace port and consists of a pyrolitic boron nitride (PBN) crucible with a gas feed line on one end and a PBN aperture plate on the other. High purity $(7 \mathrm{~N})$ nitrogen gas is flowed through the source during operation. An electrical discharge is established in the gas by an inductively-coupled $13.56 \mathrm{Mhz} R \mathrm{R}$ excitation. This discharge serves to generate a plasma in the $\mathrm{N}_{2}$ within which, a fraction of the molecular nitrogen is dissociated. Under the continuous flow condition present in this source, a variety of species escape the source through the aperture plate into the vacuum environment. This resulting flux can conceivably contain a mixture of atomic and molecular nitrogen and ions of each. A thorough characterization of this flux is presented in chapter four.

The Oxford source can operate with up to $600 \mathrm{~W}$ of RF power coupled into the plasma. The source is equipped with an optical port on axis with the plasma chamber through which it is possible to monitor the optical emission from the plasma with a photodiode and voltmeter. The source temperature is maintained by flowing dry, cryogenic $\mathrm{N}_{2}$ gas, which is boiled off from a liquid $\mathrm{N}_{2}$ dewar, around the plasma chamber and RF coil. The length of time for which a continuous GaN growth can occur is currently limited by the available cooling gas supply at a given RF power input.

\begin{tabular}{|c|c|c|c|c|}
\hline \multicolumn{5}{|c|}{ PBN Aperture Plates for CARS25 Source } \\
\hline Aperture & Application & $\begin{array}{c}\text { Conductance } \\
(\mathrm{l} / \mathrm{s})\end{array}$ & \# of holes & $\begin{array}{c}\text { Diameter of } \\
\text { holes (mm) }\end{array}$ \\
\hline 1 & Doping & 0.5 & 9 & 0.2 \\
\hline 2 & Doping & 0.5 & 1 & 0.6 \\
\hline 3 & GaN Growth & 10 & 37 & 0.5 \\
\hline 4 & GaN Growth & 10 & 9 & 1.0 \\
\hline 5 & GaN Growth & 10 & 1 & 3.0 \\
\hline 6 & GaN Growth & 10 & 255 & 0.2 \\
\hline
\end{tabular}

Table 3.1 Aperture plates available for the CARS25 Nitrogen source 
The nitrogen source comes with two PBN aperture plates: one providing a low gas conductance $(0.5 \mathrm{l} / \mathrm{s})$ for doping fluxes, the other providing a higher conductance $(10 \mathrm{l} / \mathrm{s})$ for higher output flux. Additional plates with these conductances but varying geometries were manufactured by Angstrom Sciences (Pitt. PA) as part of the source characterization described later. Table 3.1 lists the attributes of the available aperture plates.

For a given aperture conductance and RF power, a stable plasma can be generated and maintained only within a narrow range of source inlet pressures.

\subsection{Thin Film Characterization}

\subsubsection{Hall effect}

Use of the Hall effect for semiconductor characterization provides a quick and inexpensive measure of a sample's electrical characteristics. Simple voltage measurements in the presence of a magnetic field provide important information on the density and sign of free carriers and also their mobility. Samples grown for this study were characterized in a room-temperature Hall effect system in the WVU MBE lab. A 6 kilo-gauss electromagnet is used to provide the DC field with a Keithley Model 220 programmable current source to provide the input current. A Keithley Model 7001 Switch box and Model 7065 Hall Effect Card provide the switching circuits used to average out contact and thermal effects. The Hall voltage is measured by a Keithley 197A digital voltmeter (DVM). System control and data acquisition are performed by an IBM PC over an IEEE-488 control bus (Figure 3.7).

Samples were measured using the Van Der Pauw 4-point geometry [20] which allows measurement of samples of arbitrary shape so that no post growth processing is required before measurement. Electrical contact was made by soldering wires directly to the GaN with indium. In general, all electrical contacts to semiconductors are rectifying 


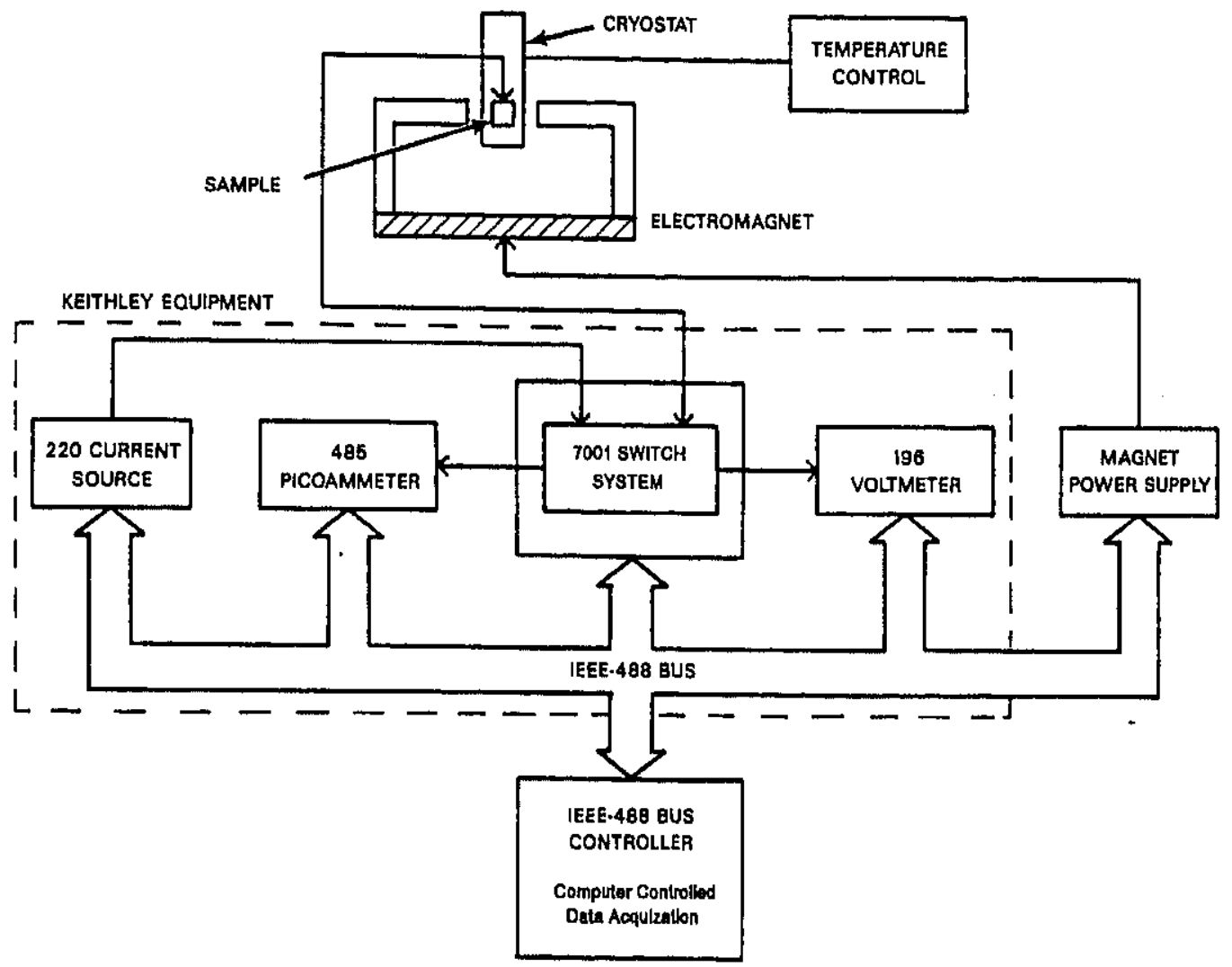

Figure 3.7 Schematic of a Hall measurement setup. 
in nature so it is important to find an operating current which falls within the linear, ohmic region of the contact's current vs. voltage (I-V) curve. With this established, the computer is allowed to collect and average a large sample of voltages for each of the possible current and voltage contact permutations. These values are averaged and used to define the resistivity and Hall voltage. The Hall voltage is directly proportional to the Hall coefficient $\mathrm{R}_{\mathrm{H}}$. Once these values have been calculated, one can calculate the carrier concentration and mobility by the following equations.

$$
\begin{aligned}
& n=\left(e R_{H}\right)^{-1} \\
& \mu=\frac{R_{H}}{\rho}
\end{aligned}
$$

\subsubsection{Atomic Force Microscopy}

Atomic force microscopy(AFM) was created in answer to the scanning tunneling microscope's inability to image a non conducting surface. AFM is a surface profiling technique where a sharp probe is scanned across the sample surface and the resultant probe deflection is measured $[21,22]$. This probe deflection can then be visualized either as the force between the sample and probe tip or as a change in the sample to probe tip distance which is converted into a relative height change. (See Figure 3.8) The AFM studies performed here were conducted in the lab of Prof. Naresh Dalal within the WVU Dept. of Chemistry by both the author and Dr. Michelle Richards-Babb using a Digital Instruments NANOSCOPE II AFM system. This open-air system was used primarily to assess large-scale surface morphology, at sub-optical resolution. 


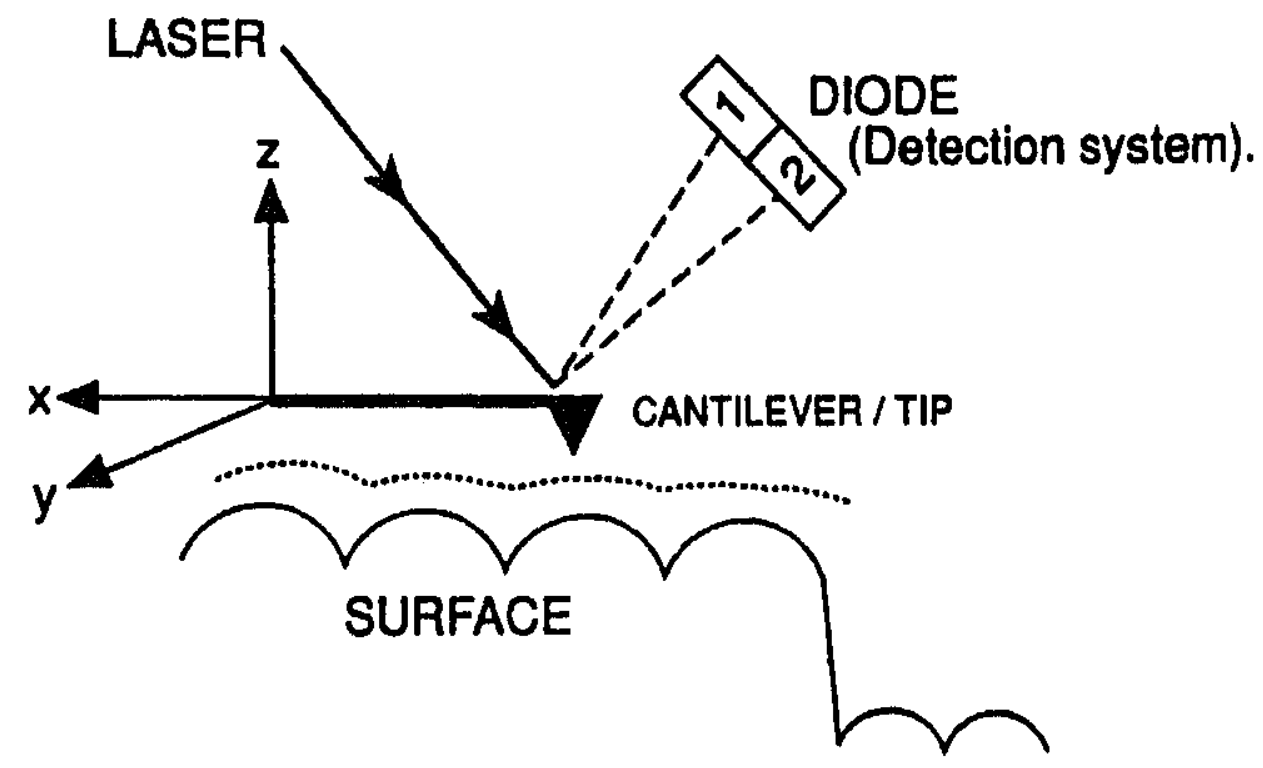

Figure 3.8 Schematic of the atomic force microscopy measurement system. 


\subsubsection{Photoluminescence Spectroscopy}

Many types of semiconductor spectroscopies are based on the fact that a fraction of excess electrons and holes decay into lower level states by emitting a photon of light with the wavelength determined by the energy change between the initial and final states. By exciting a semiconductor with above-bandgap light to create excess holes and electrons and then analyzing the light emitted by the sample, it is therefore possible to identify radiative energy levels related both to the bandgap and to defects and impurities. PL studies were conducted by Prof. Nancy Giles in her laboratory within the WVU Physics Department on several samples grown during this work. Above bandgap excitation was provided by the $325 \mathrm{~nm}$ line of a He-Cd laser focused to a nominal power density of $5 \mathrm{~W} / \mathrm{cm}^{2}$. PL was collected with a grating monochromator and a GaAs cathode photomultiplier. The input beam is optically chopped and the PL signal is run through a lock-in amplifier to increase the signal-to-noise ratio. For comparison to our results, a table of typical PL lines seen by other researchers is shown below [14]. 


\begin{tabular}{cc}
\hline Low Temperature GaN Luminescence Peaks \\
Free excitons & $3.474-3.475 \mathrm{eV}$ \\
& $3.480-3.481 \mathrm{eV}$ \\
& $3.49 \mathrm{eV}$ \\
Free exciton LO replica & $3.385 \mathrm{eV}$ \\
Free exc ton 2-LO replica & $3.293 \mathrm{eV}$ \\
Donor bound exciton (I 1$)$ & $3.44-3.47 \mathrm{eV}$ \\
LO replica & $3.377-3.378$ \\
2-LO replica & $3.286 \mathrm{eV}$ \\
TO replica & $3.400 \mathrm{eV}$ \\
Acceptor bound exciton & $3.455 \mathrm{eV}$ \\
Cd-acceptor bound exciton & $3.454-3.455$ \\
LO replica & $3.364 \mathrm{eV}$ \\
2-LO replica & $3.355 \mathrm{eV}$ \\
Donor-acceptor & $3.26-3.27 \mathrm{eV}$ \\
LO replica & $3.17-3.18 \mathrm{eV}$ \\
2-LO replica & $3.08-3.09 \mathrm{eV}$ \\
3-LO replica & $2.99-3.00$ \\
\hline
\end{tabular}

\subsubsection{Transmission Electron Microscopy}

A sample was characterized by transmission electron microscopy (TEM) by Dr. Linda Romano at XEROX Palo Alto Research Center (Palo Alto, CA). The sample was investigated by a combination of high resolution transmission electron microscopy, multiple dark field imaging and convergent electron beam diffraction techniques. The sample was cut parallel to the growth direction and then polished to a thickness of less than $5 \mu \mathrm{m}$ for electron transparency. 


\subsubsection{Secondary Ion Mass Spectrometry}

Two samples were characterized by means of secondary ion mass spectrometry at Charles Evans and Associates (Redwood City, CA). By sputtering away sample material with an $8 \mathrm{keV} \mathrm{O}_{2}{ }^{+}$ion beam and monitoring the sputtered material with a Cameca magnetic sector mass spectrometer, a depth profile was developed for boron, potassium and lithium in the GaN samples.

\subsubsection{UV Fluorescence Microscopy}

This technique is similar to the photoluminescence spectroscopy described earlier in that UV light is used to excite lower energy radiative transitions in the sample. Instead of providing detailed spectral information, this technique provides spatial information for the source of the PL. Some spectral information is provided, however, by apparent color variations. Studies were performed using an Olympus BX60M microscope located in the WVU MBE lab. The microscope was equipped with a dichroic beam splitter to allow the output of a 100W Hg lamp to pass to the sample while allowing only visible and near-UV $(380 \mathrm{~nm})$ light to be transmitted to the eyepiece or camera. 


\section{4. $\mathrm{N}_{2}$ Source Characterization}

For low temperature MBE growth of $\mathrm{GaN}$, it is necessary to find a non-thermal method for breaking the $9.5 \mathrm{eV}$ bond of molecular nitrogen. This has been attempted in a number of different ways. To date the most successful methods in the MBE arena have been to use either plasma sources, both RF and electron cyclotron resonance (ECR), or catalytic decomposition of ammonia on the GaN surface. The use of ammonia, though, brings in the problem of hydrogen, which can negate the benefits of the clean MBE environment over CVD processes. Plasma sources, on the other hand, allow control over the growth environment to be maintained. One question that remains, however, is the identification of the species produced by the source. It is likely that the flux contains both atomic and molecular nitrogen, possibly in a variety of excited states, and ions of each. Most researchers agree that it is neutral nitrogen which is responsible for $\mathrm{GaN}$ growth in MBE. Some groups have, however, discussed the possible benefits of low energy nitrogen ions [9] and also the detrimental effects of high energy ions [10]. It is important then that we understand what species are produced by our source in order to better define the growth parameters which lead to high quality film growth. In order to answer these questions, an optical and mass spectral analysis has been performed with the assistance of Prof. Charter Stinespring and his lab within the WVU Department of Chemical Engineering [23].

\subsection{Characterization Technique}

\subsubsection{Mass Spectrometry}

The Oxford Applied Research CARS25 source was installed in an UHV vacuum system in the WVU Chemical Engineering Department in direct line of sight with an Extranuclear Labs (Extrel) quadrupole mass spectrometer. The distance from the source 
aperture plate to the ionizing volume of the mass spectrometer was approximately $8 \mathrm{~cm}$. This is shown schematically in Figure 4.1. The chamber was pumped by one or more turbomolecular pumps (TMP) to a base pressure of roughly $10^{-9}$ Torr. The mass spectrometer itself was differentially pumped by a TMP to similar base pressures and maintained a pressure roughly two orders of magnitude lower than the test chamber during source operation.

The Extrel mass spectrometer consists of an ionizer, a set of electrostatic lenses, a quadrupole mass filter and an electron multiplier, shown schematically in Figure 4.2. A filament in the ionizer generates an electron beam which ionizes species within the ionizer volume. The ions created are then propelled into the lenses and mass filter by an electrostatic potential, henceforth called the ion energy, created between the ionizer and the mass filter. The ion energy potential also serves to repel external ions which have energies less than the set ion energy. Before entering the filter, the ions are focused into a beam by the lens array.

Once an ion beam has been created and focused, it needs to be separated and analyzed as a function of species mass. This is accomplished in the quadrupole mass filter. The Extrel mass filter consists of four conductive parallel hyberbolic cylindrical surfaces, arranged on the four corners of a cross, to which an RF voltage is applied. Opposing electrodes are connected together with one pair taking a potential

$$
U(t)=U+U_{1} \cos (\omega t)
$$

and the other pair taking $-\mathrm{U}(\mathrm{t})$. Ions entering the quadrupole with momenta roughly parallel to the z-axis undergo transverse oscillations in response to the ac and dc fields imposed in the $x-y$ plane. For a given selection of $U$ and $U_{1}$, only ions with a specific mass to charge ratio will experience a stable oscillation and traverse the filter to be counted. Ions which do traverse the filter strike an electron multiplier generating a current which is measured as the signal intensity. By varying the ac and dc voltages in the filter 


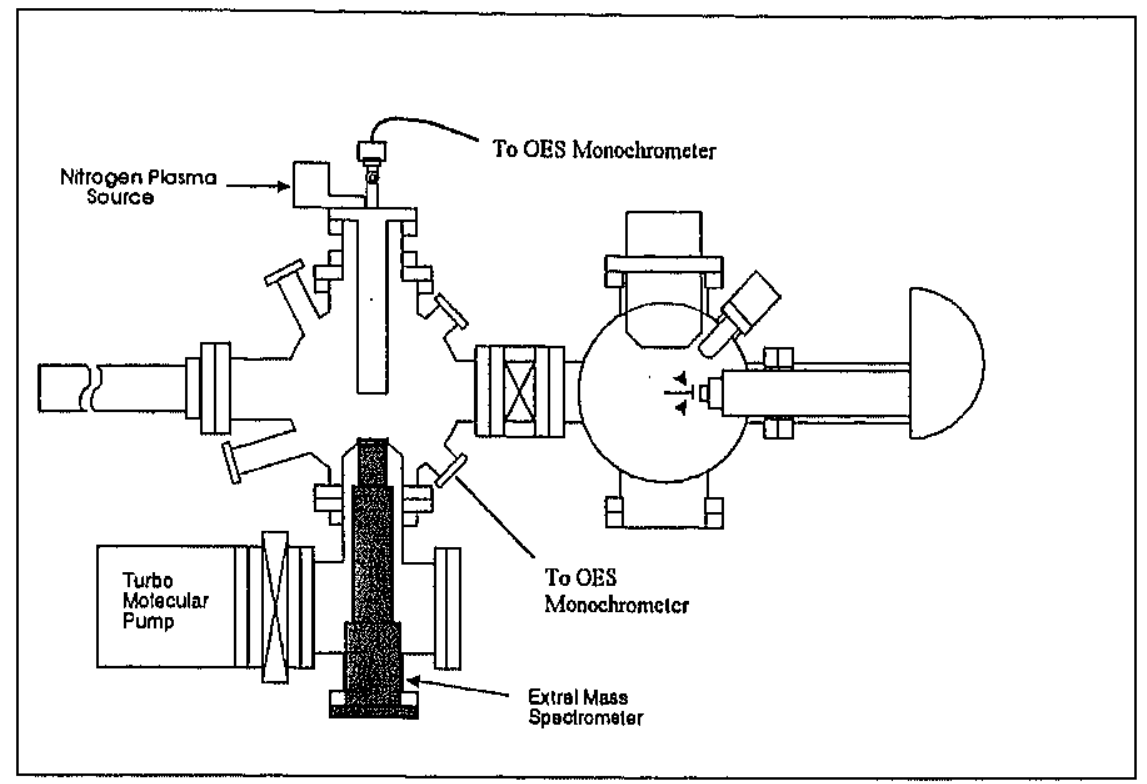

Figure 4.1 Schematic of nitrogen source characterization chamber. Source is mounted down the mass spectrometer line-of-sight. The distance from the source to the mass spec. is roughly $8 \mathrm{~cm}$. 


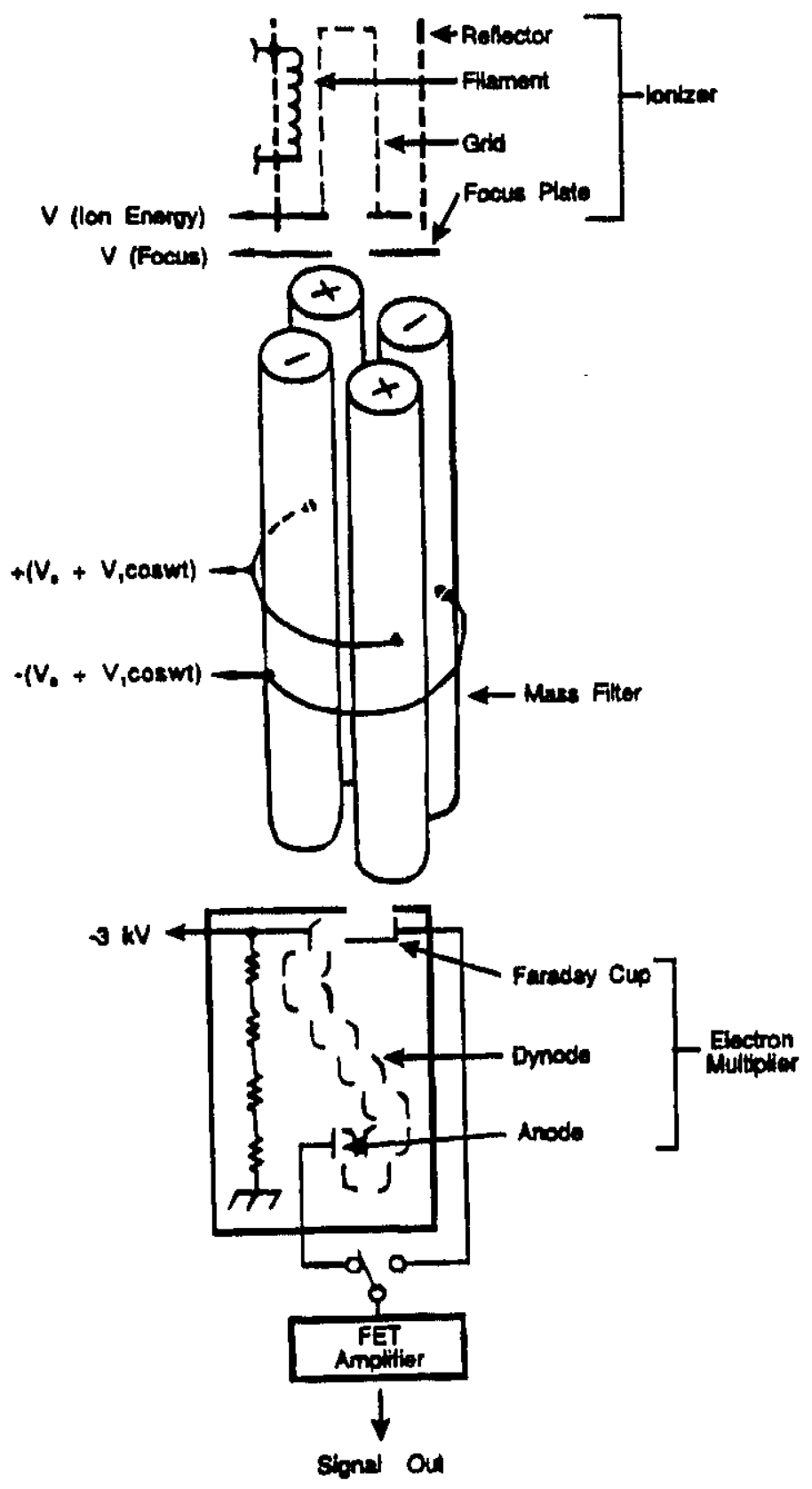

Figure 4.2 Schematic diagram of QMS system. 
and monitoring the signal intensity, it is possible to generate a spectrum for a range of mass to charge ratios.

The fact that the quadrupole filter measures the charge to mass ratio and not the mass directly immediately poses one problem for the current research. The purpose of this characterization was to differentiate between $\mathrm{N}$ and $\mathrm{N}_{2}$ and ions of each in the source output flux. For $\mathrm{N}^{+}$and $\mathrm{N}_{2}{ }^{+}$, this is easily accomplished but, if $\mathrm{N}_{2}^{++}$should be present, it would be indistinguishable from $\mathrm{N}^{+}$. For ions created in the mass spectrometer (neutrals in the source output flux), it was sufficient to keep the energy of the ionizer electron beam no higher than $20 \mathrm{eV}$. This energy is sufficient to efficiently ionize all species of interest without being high enough to dissociate or doubly-ionize the $\mathrm{N}_{2}$.

Figure 4.3 shows the transmission function for the quadrupole filter as a function of ion energy for both atomic and molecular nitrogen. This figure was produced, with the source turned off and a fixed background of $\mathrm{N}_{2}$, by adjusting the electron energy of the ionizer up to $70 \mathrm{eV}$. The resulting electron impacts have enough energy to dissociate some of the molecular nitrogen. A mass spectrum was then compiled for both the atomic and molecular peaks as a function of ion energy. The atomic nitrogen transmission peak is shifted to slightly lower energy compared to the $\mathrm{N}_{2}$. We believe that this is due to $\mathrm{N}^{+}$ ions acquiring a higher velocity than the $\mathrm{N}_{2}{ }^{+}$in the ionization process. For subsequent analysis, the transmission functions for $\mathrm{N}$ and $\mathrm{N}_{2}$ are taken to be the same. Similar transmission functions were obtained for each source aperture and operating pressure and were later used to correct the energy distribution data.

Since the source is likely generating an ionic component to its flux, it was necessary to be able to discriminate the ionic contribution from the source and that generated in the mass spectrometer. This was possible by operating the mass spectrometer in two modes, low emission and high emission. In low emission operation, the filament current is reduced to $0.018 \mathrm{~mA}$ effectively stopping ion production in the mass spectrometer ionizer. Any signal measured in the system must then be due to ions 


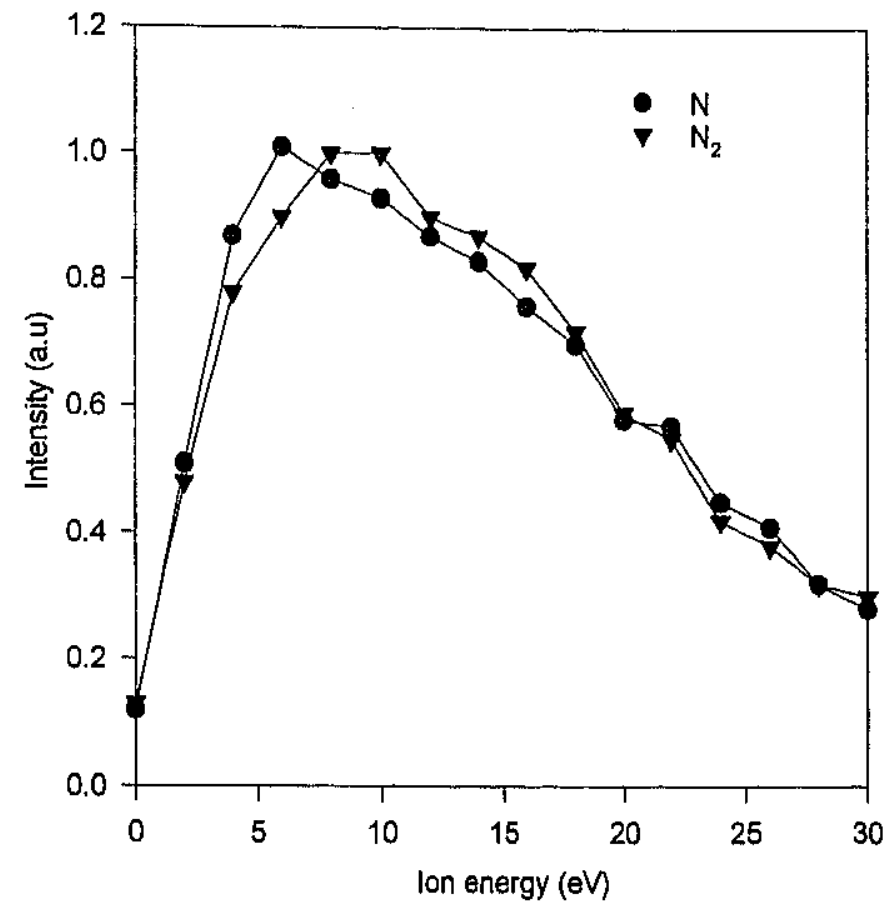

Figure 4.3 Mass filter transmission function for $\mathrm{N}$ and $\mathrm{N}_{2}$ 
originating outside the ionizer. In high emission operation, the filament current is turned up to $1 \mathrm{~mA}$ to so that ions are generated in the ionizer. The measured signal is now a combination of the signals from external and internal ion sources. By subtracting the low emission data from the high, one gets the signal due to neutrals in the source flux. This data is then adjusted to the transmission function and scaled to the ionization fraction of the particular species.

\subsubsection{Optical Emission Spectroscopy}

The CARS25 source is equipped with an optical emission monitor which uses a fiber optic cable to sight down the axis of the source to monitor plasma light output. It has been suggested that this monitor might be used as a process indicator to fine tune the output for maximum atomic nitrogen flux. Source characterizations performed by other labs [24-28] have used spectral analysis of this output to determine the composition of the output flux. It was hoped that the output of the optical monitor could be matched to the mass spectral data to provide some useful control information.

The principle behind optical emission spectroscopy is that an excited molecule will eventually radiatively de-excite emitting a photon with a wavelength dictated by the change in energy between the excited and 'ground' states. Typically, these energy states are unique and provide a fingerprint for the identity of the radiating species. By monitoring the source's optical output with a monochrometer, it is possible to extract these 'fingerprints' and see how they vary as a function of the source operating conditions.

Light output was monitored via a fiber optic cable initially connected to the optical port at the rear of the plasma source. This was later moved such that the fiber viewed the exit aperture at the front of the source (Refer back to Figure 4.1). In normal operation, this light is monitored by a silicon photodiode and the integrated intensity is displayed on a digital voltmeter(DVM). For this study, the light was passed into an Instruments SA, Inc. HR-320 Spectrograph. The HR-320 has a $0.32 \mathrm{~m}$ focal length and 
was operated with a 1200 grooves $/ \mathrm{mm}$ grating. A stepper motor under computer control drives the spectrograph to scan through a wavelength region. The spectrograph output was monitored by a Si photo-voltaic detector, which was read by computer through an IEEE-488 interface on a Keithley 197 DVM. Scans could then be saved for later analysis. A typical scan is shown in Figure 4.4.

Vaudo et al. [26,27], performed high resolution spectral scans on a similar nitrogen source and showed conclusively that the peaks at 745,821 , and $869 \mathrm{~nm}$ are due to transitions in atomic nitrogen. These peak assignments were used here for the spectral analysis. Peaks were fit using Jandel Scientific Peakfit non-linear curve-fitting software. All peak heights were normalized to the $773 \mathrm{~nm}$ molecular line so that a comparison could be made between the relative molecular and atomic peak intensities.

\subsection{Results}

Analysis was performed on four source aperture plates described in section 3.1.4. Apertures 1 and 2 are typically used for doping applications requiring a low flux. Apertures 3 and 4 are representative of the aperture used for $\mathrm{GaN}$ growth.

During typical operation of this source, a silicon photodiode monitors the integrated light intensity from the plasma chamber. The output of this detector is displayed on a digital voltmeter (DVM) and used as a primary indicator of plasma condition. Figure 4.5 shows representative plots of this photodiode response for the high and low conductance apertures used on this source. For a given RF power and aperture plate, a stable plasma can be maintained only within a narrow window of pressures. In general, the width of this existence window increases with applied power. At a fixed power, for source pressures on the low end of this existence window, the photodiode response increases linearly with increasing source pressure. The response eventually flattens out becoming relatively constant. At high enough source pressures, the response falls off again, decaying until the plasma becomes unstable. 


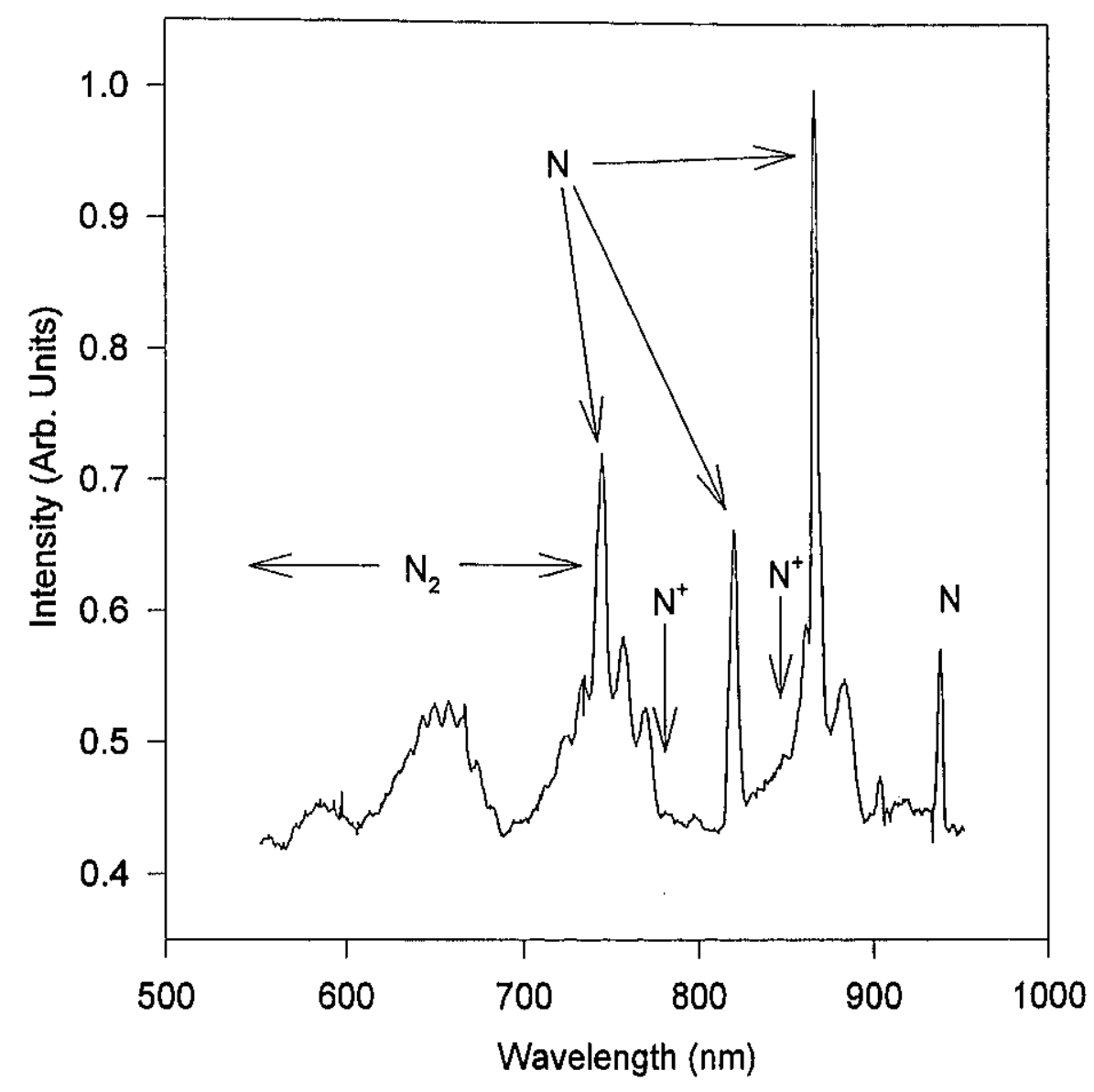

Figure 4.4 Typical spectrum of the optical emission from the CARS25 nitrogen source during operation. The labeled peaks have been related to transitions in atomic nitrogen. 

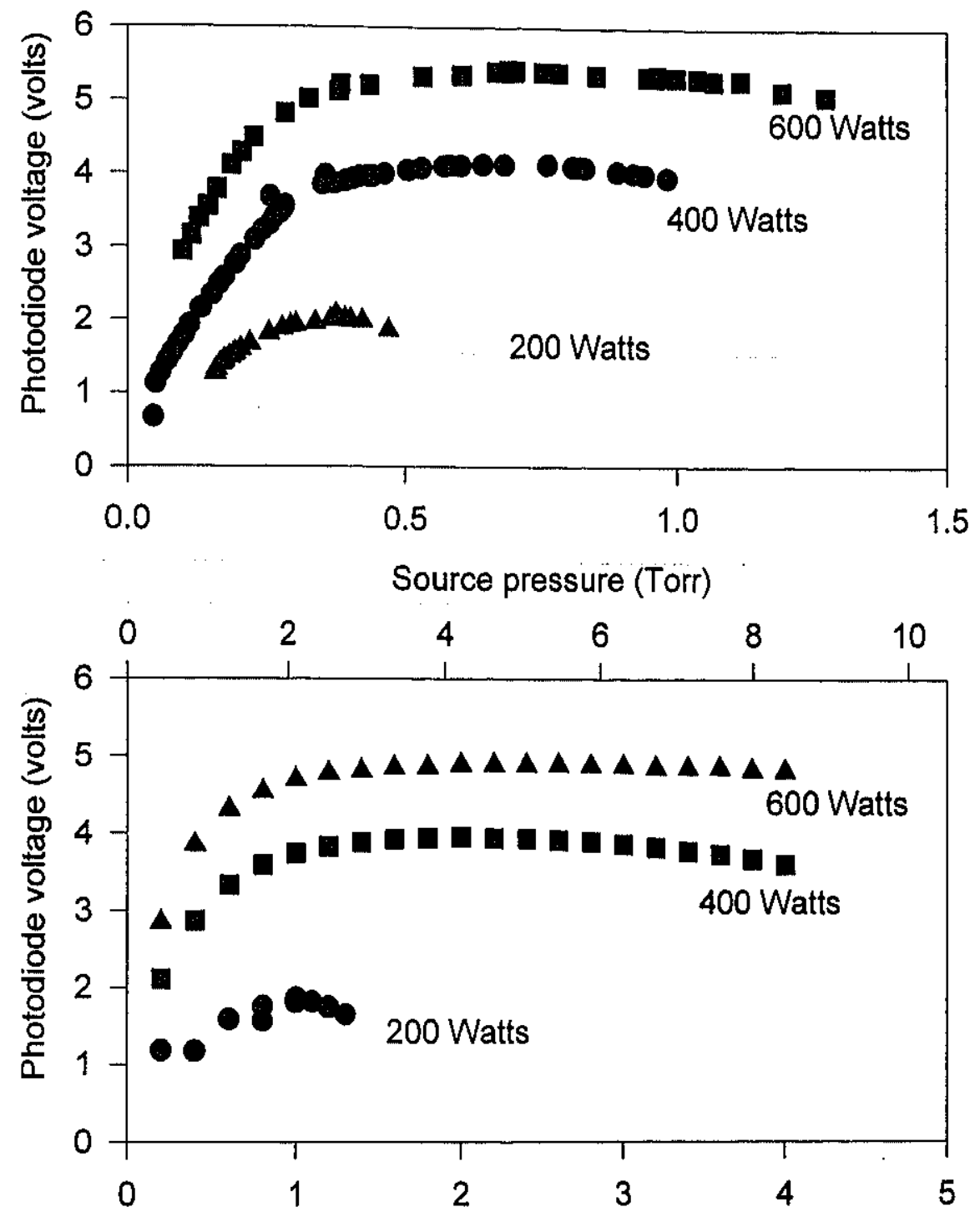

Flux through source (SCCM)

Figure 4.5 Photodiode response as a function of source pressure (Nitrogen flow for the lower plot) The upper plot is characteristic of the doping aperture, while the bottom is characteristic of the growth aperture. 

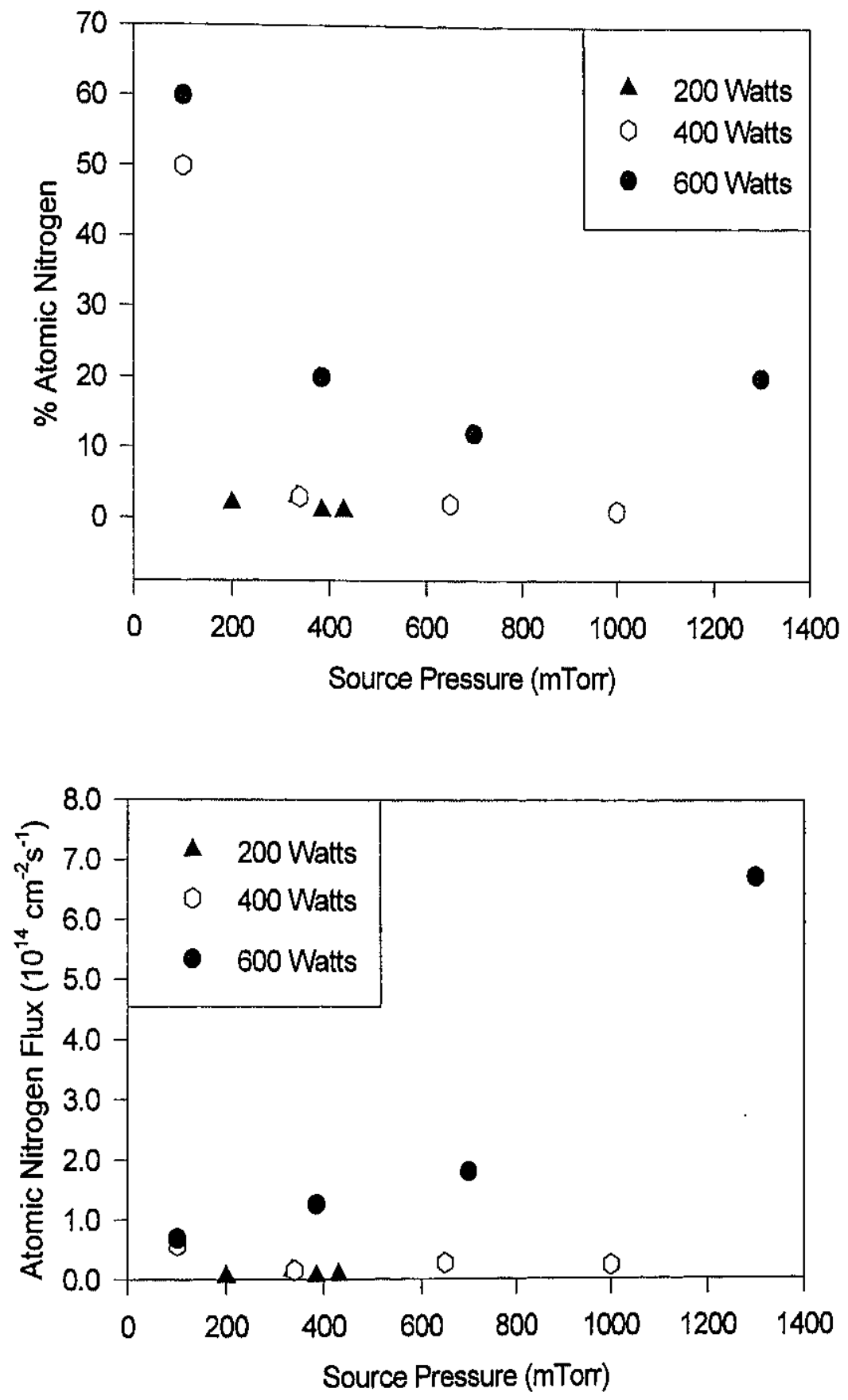

Figure 4.6 Summary plot of nitrogen source mass spectra for doping aperture \#1(top). Bottom plot shows atomic nitrogen flux at substrate. Relative error in these values is estimated to be approximately $20 \%$. 

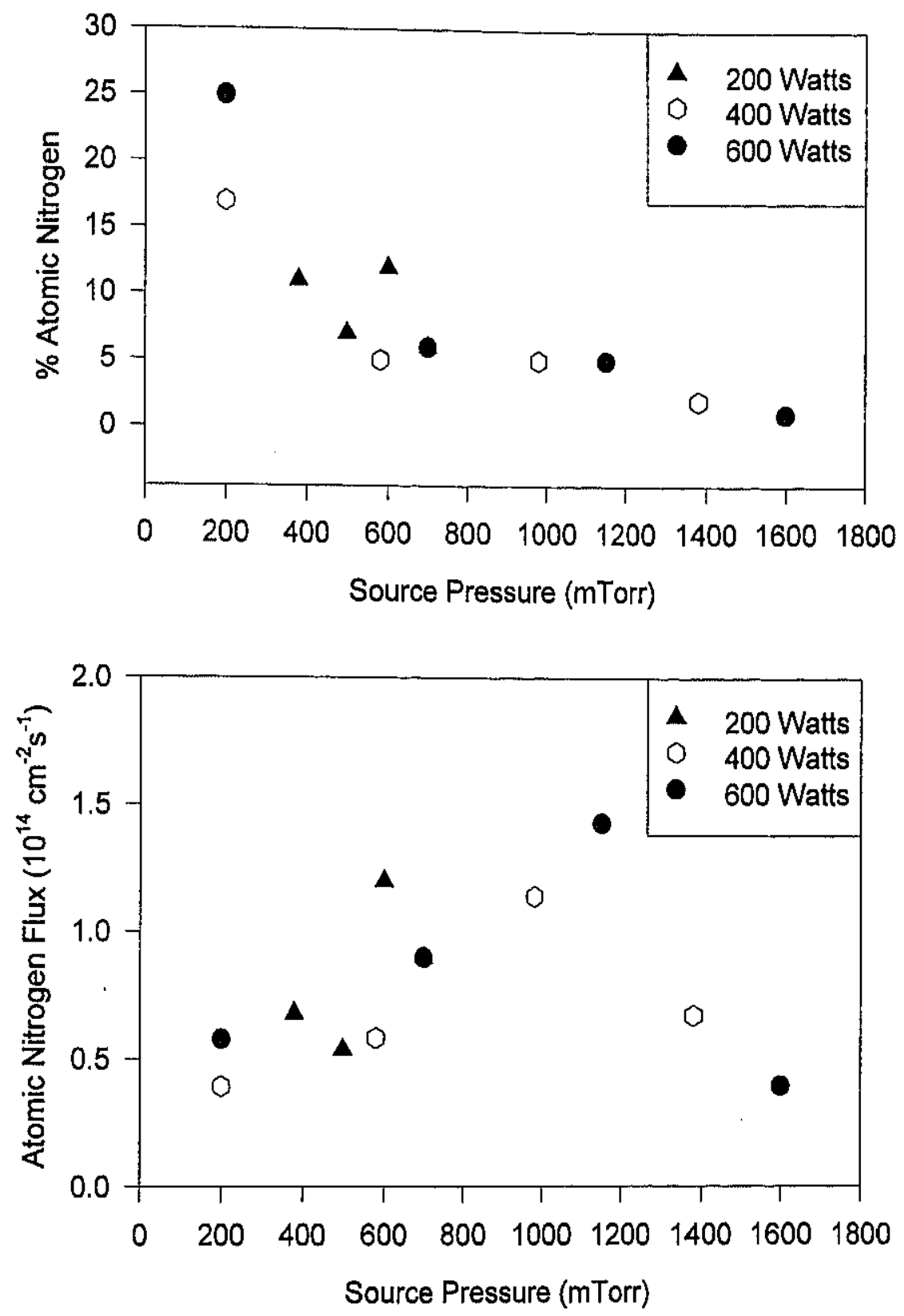

Figure 4.7 Summary plot of nitrogen source mass spectra for doping aperture \#2(top). Bottom plot shows atomic nitrogen flux at substrate. Relative error in these values is estimated to be approximately $20 \%$. 

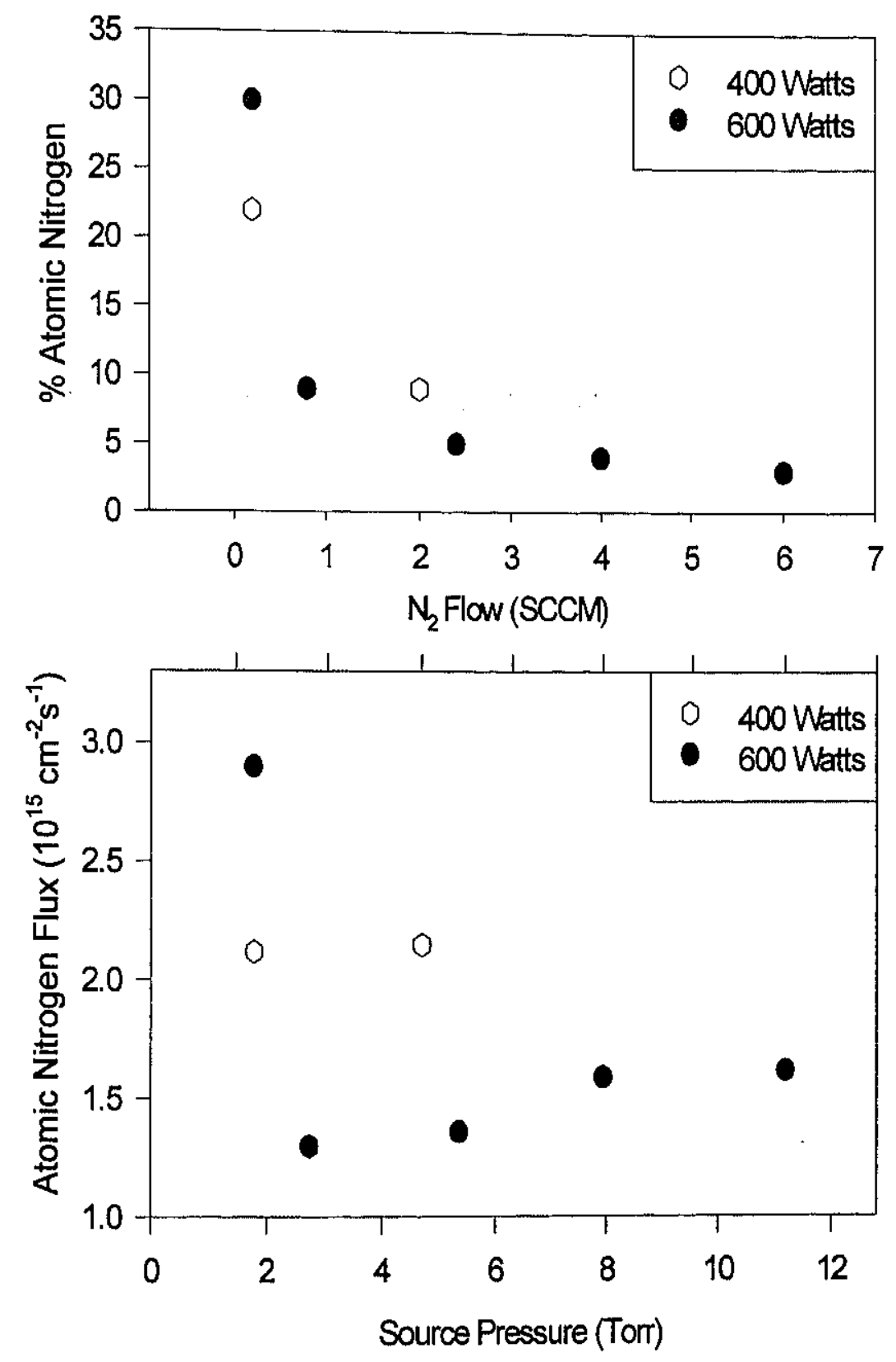

Figure 4.8 Summary plot of nitrogen source mass spectra for growth aperture \#3(top). Bottom plot shows atomic nitrogen flux at substrate. Relative error in these values is estimated to be approximately $20 \%$. 
Figures 4.6-4.8 show the results of the mass spectral analysis for apertures 1-3. In all cases, the percent conversion to atomic nitrogen decreases with increasing source pressure, at constant power. However, when scaled to actual output flux, the atomic nitrogen flux increases with pressure. Increasing power, on the other hand, is always seen to increase the fraction converted over the range investigated. From this a consistent picture begins to develop where increasing the applied power increases the energy available for molecular dissociation. Increasing the source pressure, however, increases the density of molecules and thus the probability that two atoms will collide and recombine in the source.

All of these analyses relied on the source output flux containing ions with energies lower than $50 \mathrm{eV}$. External ions with energies higher than this could not be rejected by the ionizer system and made extraction of the neutral flux difficult, if not impossible. Several points had to be rejected from these figures because a high energy ion component caused a severe overestimation of the neutral atom content. Data for aperture \#4 had to be rejected entirely because of a large flux of high energy ions coming from the source. It was impossible to extract useful information for this aperture.

From this result, it appears as though ion recombination is enhanced in the boundary layer region around the aperture plate exit holes. Larger exit holes, as designed for aperture \#4, allow more ions to go through without traversing the boundary layers and being neutralized. In order to minimize ion content in the output flux, smaller holes are necessary. For subsequent studies, an aperture plate has been made with the conductance of aperture 3 but with $0.2 \mathrm{~mm}$ holes, in the hope that this will reduce the ion flux.

Figures 4.9 and 4.10 show data from the peak fits performed on the OES data for aperture 1. All data in these figures have been normalized to the $773 \mathrm{~nm}$ molecular tine intensity. In Figure 4.9, the OES output for the atomic nitrogen lines tracks with the mass 


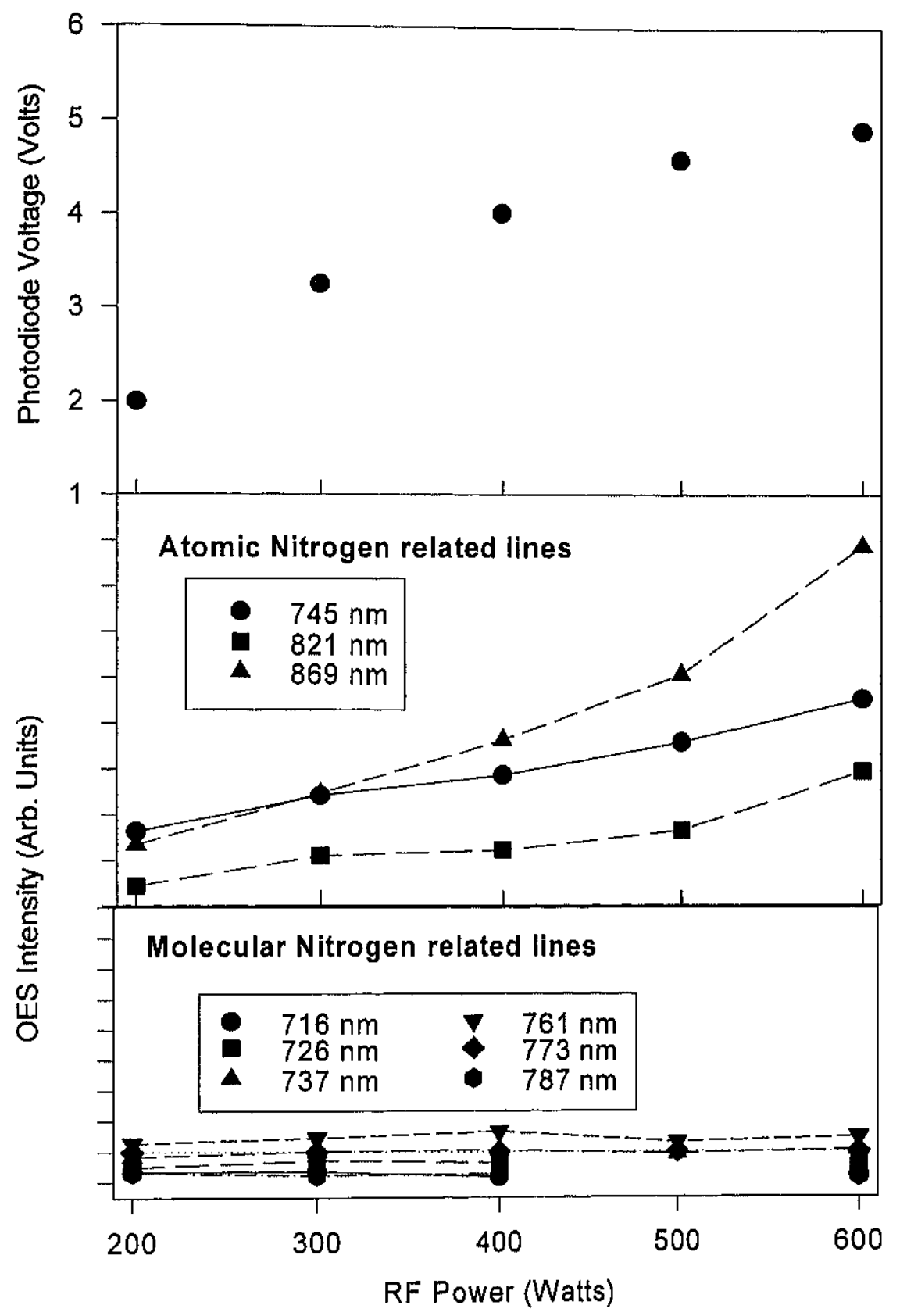

Figure 4.9 Representative plots of atomic(middle) and molecular(bottom) line intensities, normalized to the $773 \mathrm{~nm}$ line intensity, from OES peakfits at fixed source pressure. Top graph is the integrated intensity from the photodiode system included with the source. Similar results were seen for all apertures studied. 


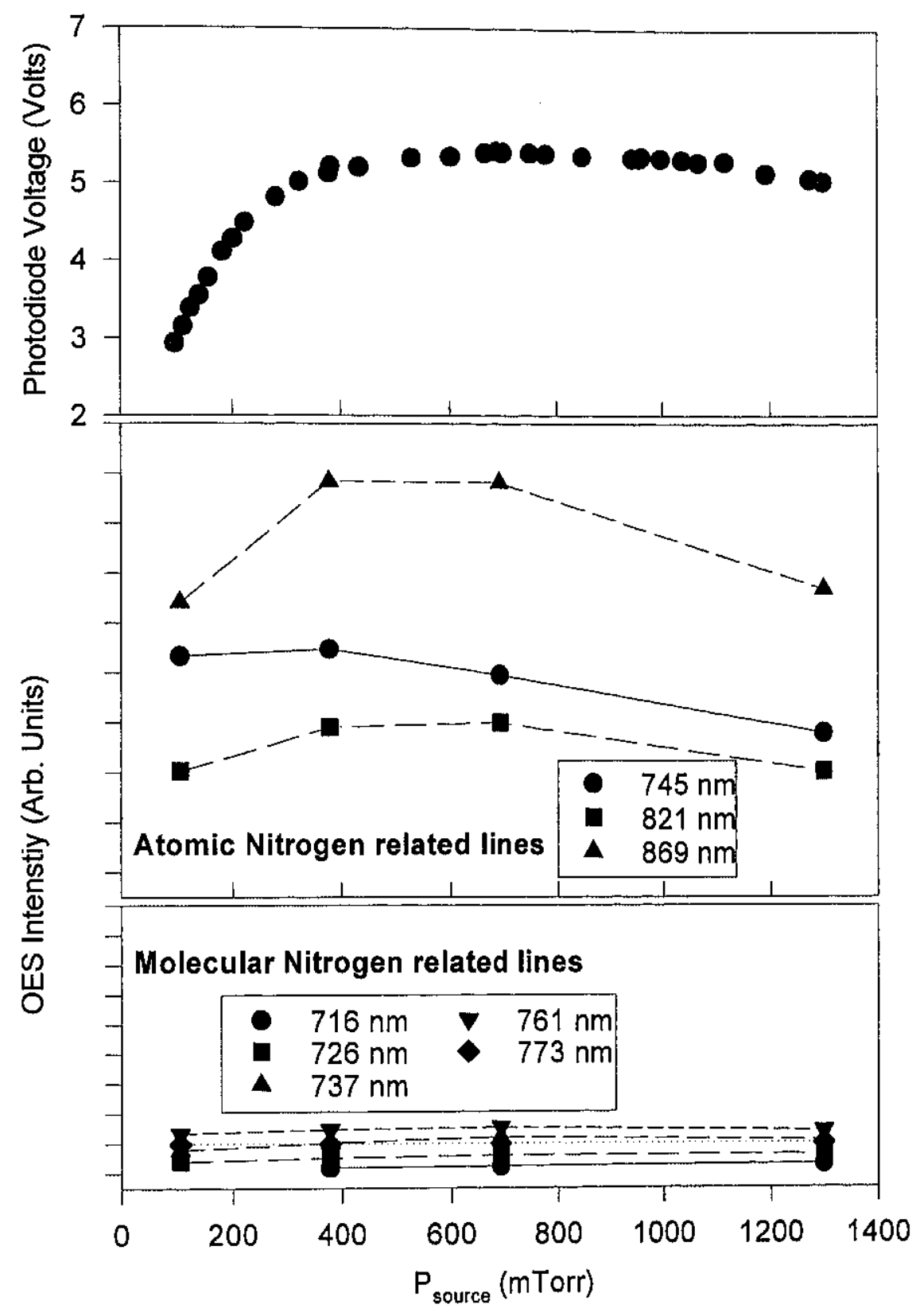

Figure 4.10 Representative plots of atomic(middle) and molecular(bottom) line intensities, normalized to the $773 \mathrm{~nm}$ line intensity, from OES peakfits at fixed source power. Top graph is the integrated intensity from the photodiode system included with the source. Similar results were seen for all apertures studied. 
spectroscopic data: as the RF power increases, so do the atomic peak intensities relative to the molecular peaks. Note that the actual intensity of both line-sets increased with power. In Figure 4.10, the atomic lines are seen to increase and then fall off again with increasing pressure. In both cases, the atomic lines seem to track well with the integrated intensity displayed at the top of each figure. These same optical trends were seen for all of the apertures tested. In all of these cases, it appears that the optical emission from the atomic lines tracks reasonably well with the integrated emission from the plasma, however, its relationship to the actual output flux content is difficult to ascertain.

For all but aperture 4, the ionic contribution to the source flux was low, about $0.03 \%$. Note, however, that this corresponds to $2 \times 10^{13} \mathrm{ions} / \mathrm{cm}^{2} / \mathrm{sec}$ and could generate a volume defect level of $10^{17} \mathrm{~cm}^{-3}$ for typical growth rates of $0.1 \mu \mathrm{m} / \mathrm{hr}$. Maximum ion energies were mostly below $25 \mathrm{eV}$. Ion energies for aperture 4 were greater than $50 \mathrm{eV}$, which is generally considered to be entirely detrimental to $\mathrm{GaN}$ growth. 


\section{Nucleation and Growth of GaN}

As discussed in section 2.2, growth of GaN on sapphire typically occurs in a three-dimensional mode, beginning with the nucleation of isolated islands that maintain their individual character during subsequent growth. Such columnar growth, due primarily to the large lattice and thermal mismatch, leads to a rough epilayer containing a high concentration of defects, which are mainly threading dislocations. The use of a low temperature GaN or AIN buffer layer, grown at $400-650^{\circ} \mathrm{C}$ and annealed at a higher temperature before resuming growth, has been shown to dramatically improve layer morphology and electrical characteristics in the grown layer.

\subsection{Nucleation and Buffer Layers}

The initial nucleation layer can dictate much of the subsequent growth quality in heteroepitaxial growth. To investigate the nucleation and initial growth of $\mathrm{GaN}$ on sapphire, a series of layers was grown under varying conditions with film thicknesses ranging from $100 \AA$ to $0.4 \mu \mathrm{m}$.

All films were grown on $1 \mathrm{~cm}^{2} \mathrm{C}$-plane (0001) sapphire substrates purchased from Union Carbide Crystal Products. Substrates were degreased in heated baths of trichlorethylene, acetone and methanol and then etched for ten minutes in a phosphoric/sulfuric (1:3) acid mixture heated to $160^{\circ} \mathrm{C}$ to remove residual polishing damage. Following this etch, the substrates were rinsed in de-ionized water (characterized by a nominal $18 \mathrm{M} \Omega$ resistance) and blown dry with high-purity $\mathrm{N}_{2}$ gas. Substrates were mounted to a molybdenum block with graphite (Aquadag) and a molybdenum mask, and placed into the chamber load-lock. After pumping out the loadlock, the sample block was transferred to the growth chamber and heated to $100^{\circ} \mathrm{C}$ for

one hour to outgas the sample and the Aquadag. Substrates were then heated to $730^{\circ} \mathrm{C}$ 
and exposed to an atomic hydrogen flux, from the thermal cracker described earlier, for twenty minutes to remove surface carbon and oxygen contamination.

Early in this study, several layers were grown using nucleation conditions reported by others $[29,16]$. Following the prevailing wisdom of GaN/sapphire growth, substrates were "nitrided" by exposure to a nitrogen plasma prior to growth. This supposedly produces a thin layer of AIN that then assists in providing strain relief for the subsequent film growth. Growth of a buffer layer on this surface resulted in a fine-grained $(<100 \AA)$ island size seen under AFM (Figure 5.1). Growth of an initial layer at low temperature $\left(450^{\circ} \mathrm{C}\right)$, without nitridization, followed by an anneal at $660^{\circ} \mathrm{C}$ also resulted in a similar small grain size (Figure 5.2). With grain diameters of this size, the resulting films are virtually guaranteed to have dislocation densities on the order of $10^{12} \mathrm{~cm}^{-2}$. This level of dislocation density has been observed in MBE material grown in many laboratories [9]. If the nucleation domains could be made larger, the effect would be to reduce this intrinsic defect concentration. As such, a series of growths was conducted to optimize the growth parameters for the nucleation layer to increase the nucleation domain (island) size. Domain size distributions were obtained by collecting ten AFM micrographs at points distributed around the sample. The islands were then approximated as circles by visual comparison with a template. From this, a histogram of occurrence frequency vs. diameter was obtained. The distributions were adequately represented by a poisson distribution, and the mean value was found by a least squares fit to the distribution (Figure 5.3). The fact that the island diameter followed a poisson distribution indicated that the nucleation process was statistical in nature.

Figure 5.4 is a compilation of these measurements for various growth conditions. As the substrate temperature is increased for a given $\mathrm{Ga}$ and nitrogen flux, the nucleation island diameter is seen to increase somewhat linearly up to some maximum island size after which island diameter decreases with increasing temperature. Increasing the Ga flux increases both the maximum island size and the maximum temperatures at which island 


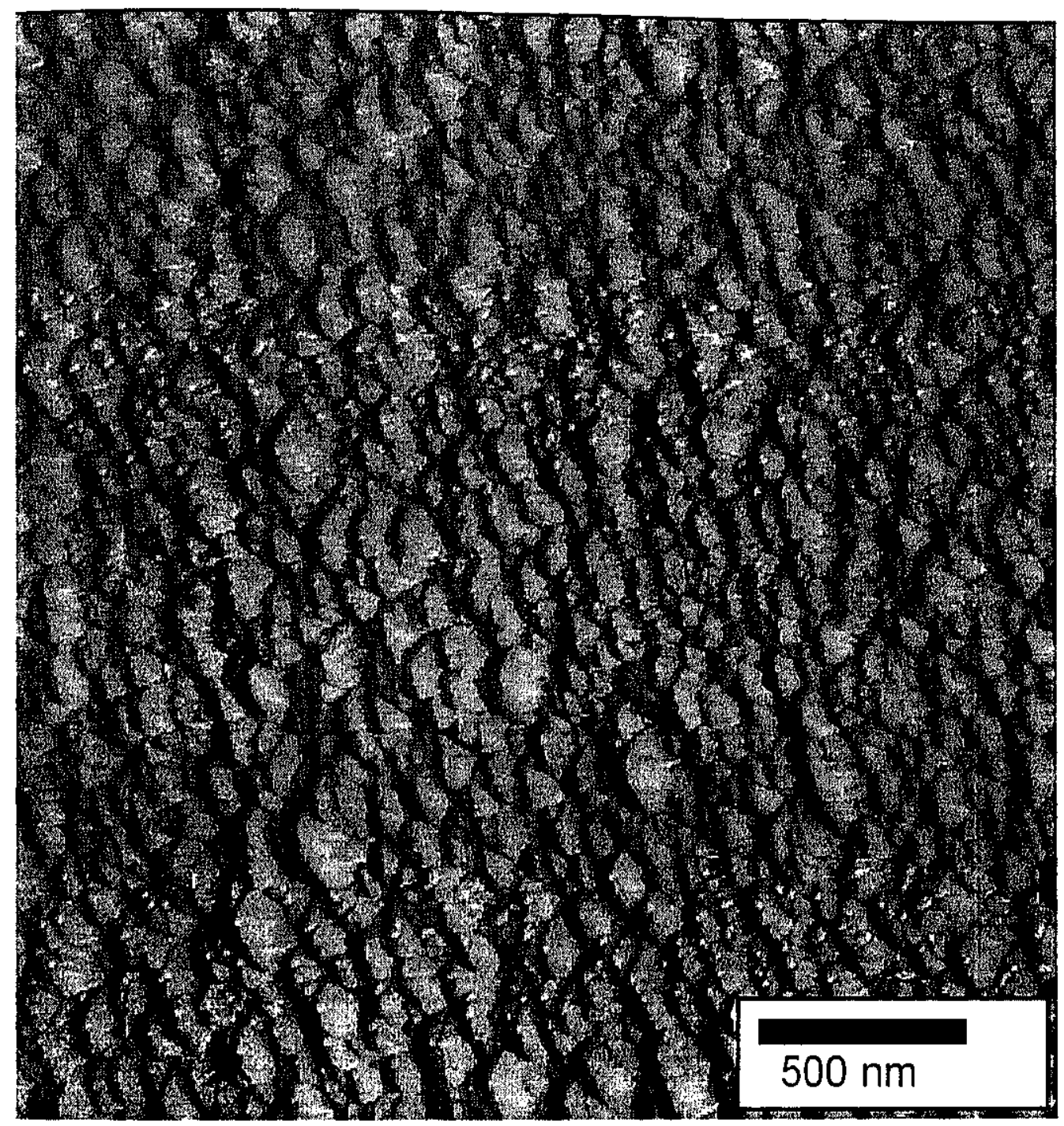

Figure 5.1 AFM micrograph of sample 9555 grown at $630^{\circ} \mathrm{C}$ substrate temperature, $2.5 \times 10^{-7}$ Torr Ga BEP, 4 SCCM N$_{2}$ and 500 watts. Sapphire was exposed to nitrogen plasma prior to growth. 


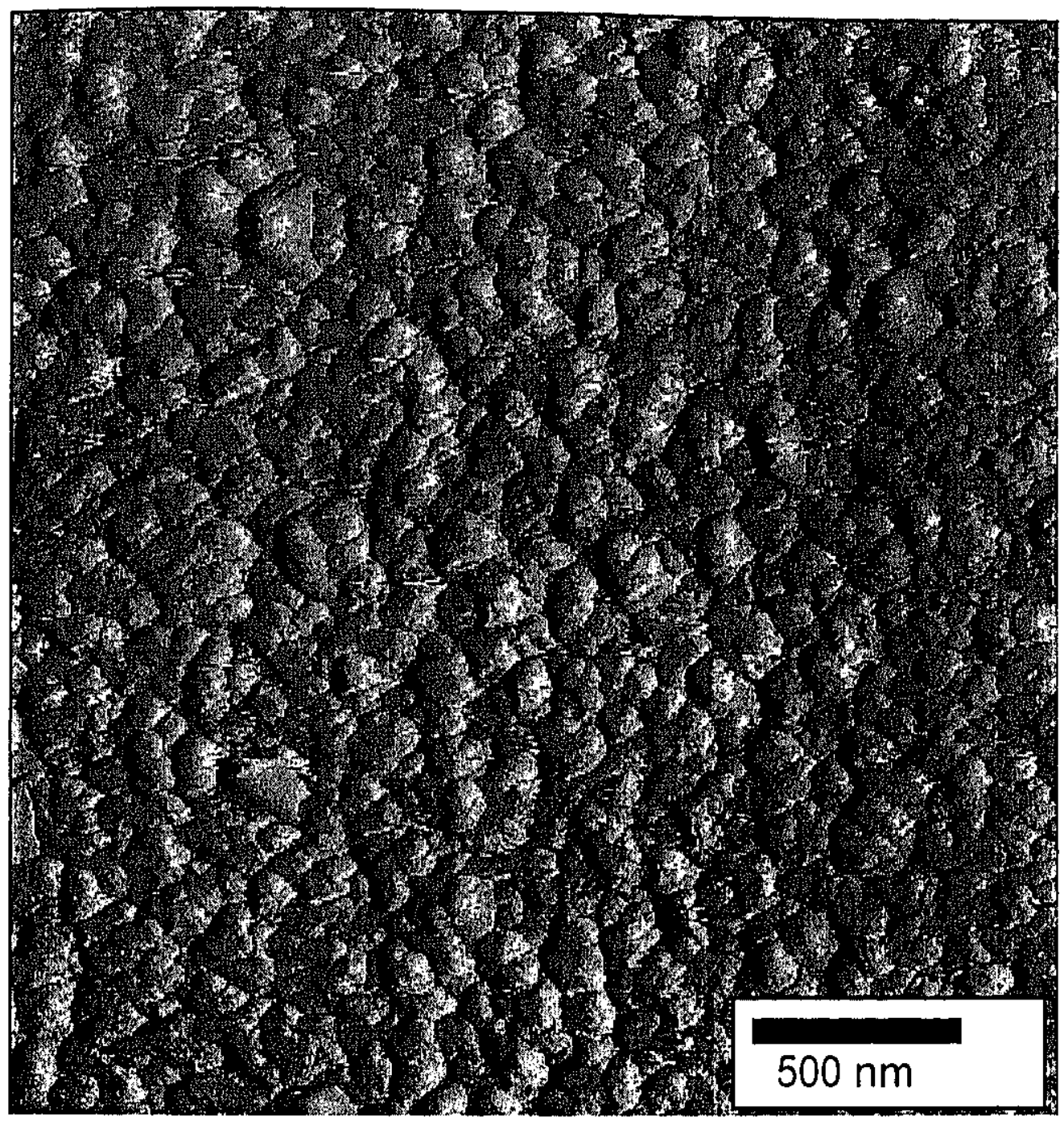

Figure 5.2 AFM micrograph of sample 9558 grown on a $50 \AA$ buffer layer grown at $450^{\circ} \mathrm{C}$ and annealed at $630^{\circ} \mathrm{C}$. Epilayer grown at $630^{\circ} \mathrm{C}$ substrate temperature, $2.5 \times 10^{-7}$ Torr Ga BEP, 4 SCCM N2 and 500W. 


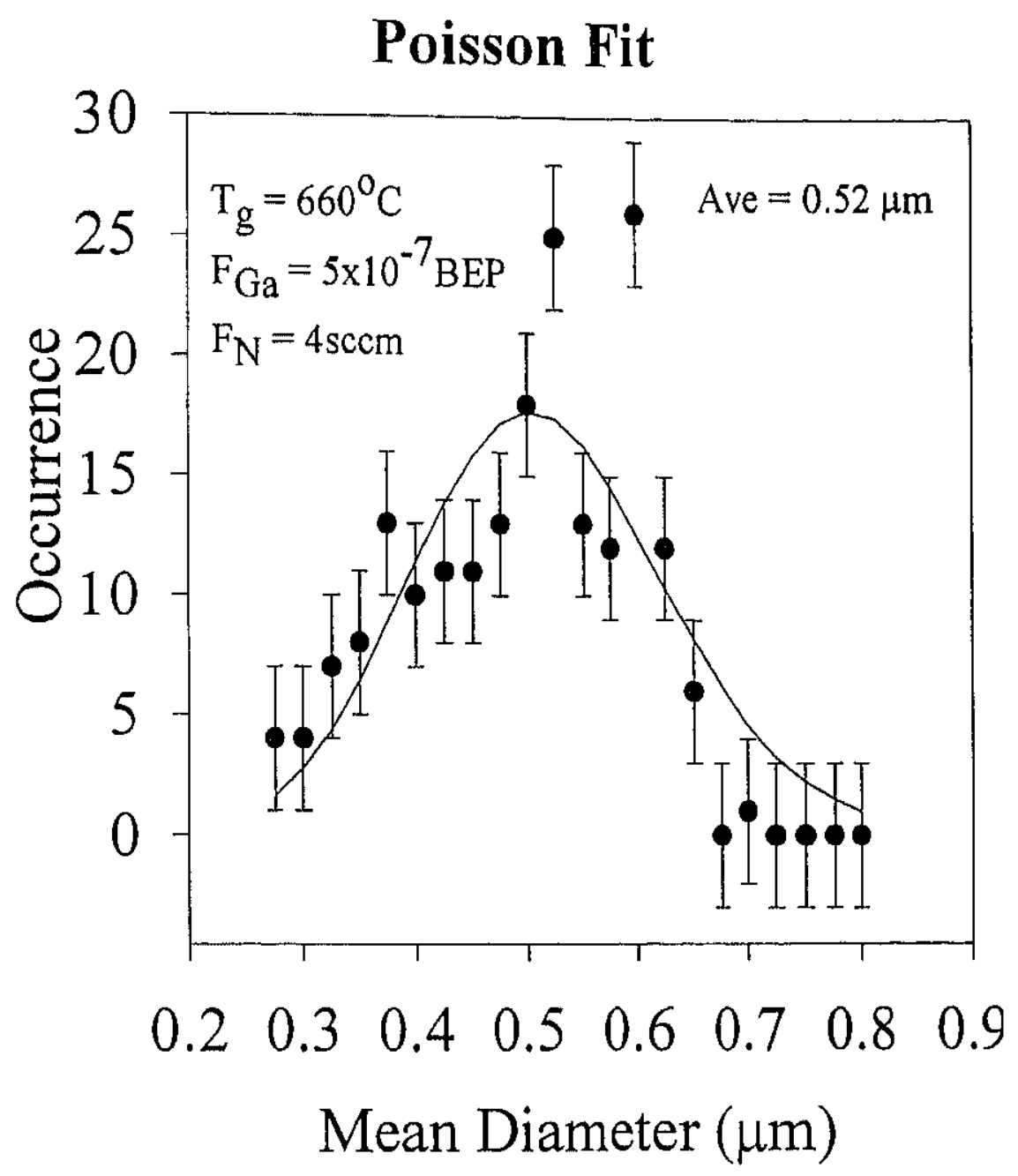

Figure 5.3 Representative fit of island diameter distribution to poisson distribution. Mean island sizes were extracted from similar fits. 


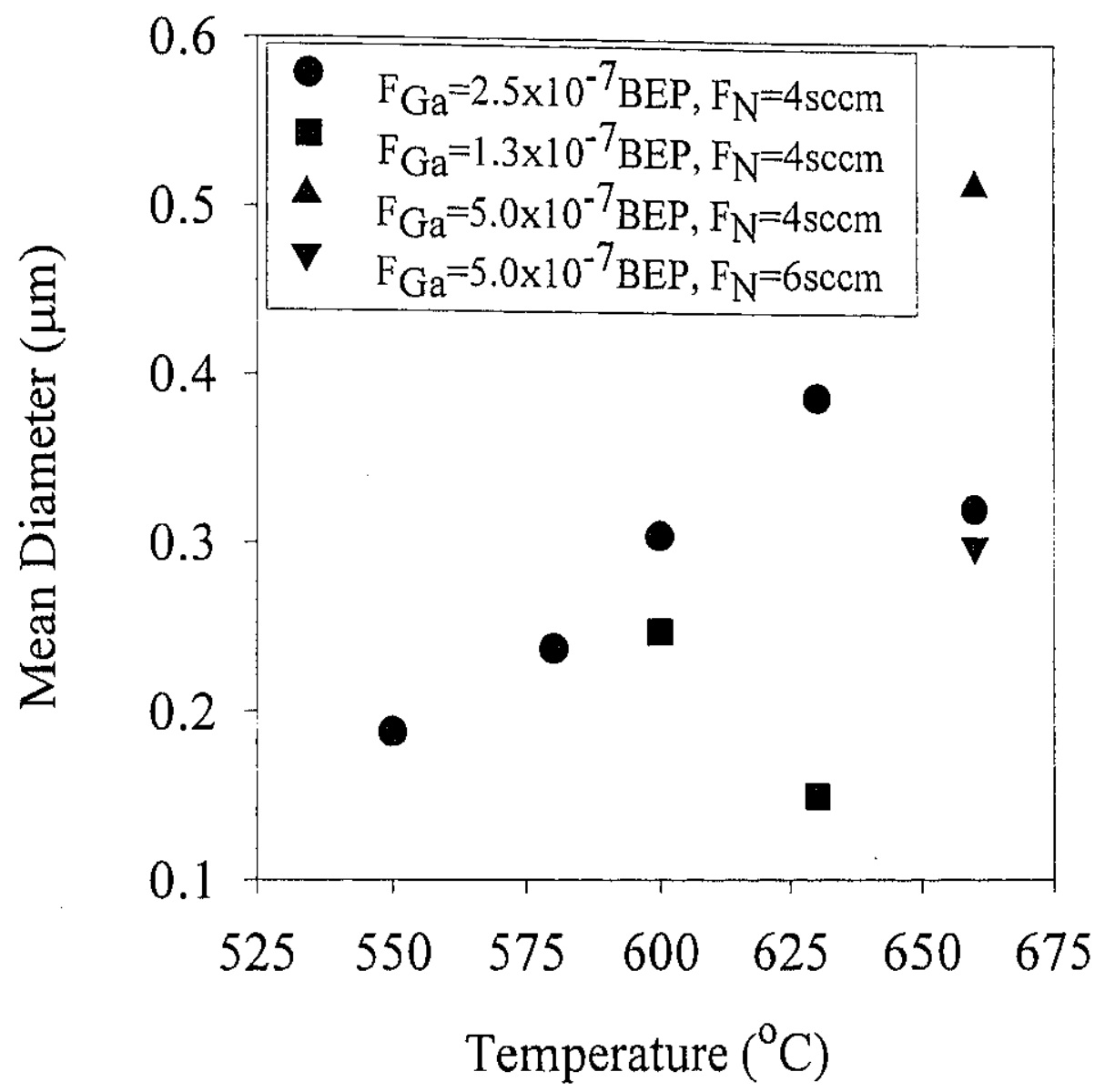

Figure 5.4 GaN nucleation island diameter as a function of growth conditions. 
diameters decrease. In contrast, increasing the active nitrogen flux decreases both the maximum island size and the temperature at which that maximum island size occurs. This seems to imply that increasing the substrate temperature for a given set of fluxes favors the growth of critically sized nucleation islands. The critically sized islands are stable to further growth and thus continue to grow, while subcritical islands either incorporate into the growing islands or evaporate. Above the maximum temperature for a given flux, the effect of increasing the temperature is similar to an increase in active nitrogen flux. The process behind this effect is not clearly understood. It is possibly related to an increase in the $\mathrm{Ga}$ desorption rate or an increase in the active nitrogen flux through the cracking of metastable nitrogen molecules at the substrate.

If we look at the growth rate of $\mathrm{GaN}$ as a function of substrate temperature and $\mathrm{Ga}$ flux (Figure 5.5) for a fixed nitrogen flux, we find that for low Ga fluxes there is a linear increase in growth rate with increasing Ga flux. This would be expected if the nitrogen flux present were sufficient to react with all of the available Ga. With further increase in Ga flux, the growth rate levels off, perhaps even declining somewhat, leading eventually to $\mathrm{Ga}$ condensation. This implies that the growth rate is limited by the availability of active nitrogen (Ga-rich growth regime). Increasing the flux of active nitrogen in this region increases the growth rate confirming this assertion. The slight decreases in growth rate over the Ga-rich region are most probably related to a transition to a more 2-D growth mode.

Figure 5.6 shows the morphological evolution for samples grown with a Ga flux of $2.5 \times 10^{-7}$ Torr at substrate temperatures up to the maximum shown in Figure 5.4. The two-fold increase in island diameter yields roughly a factor of four reduction in interisland defects that need to be smoothed out in subsequent growth. Even with the increase in domain size, the growth is still proceeding in a highly three-dimensional manner leaving a surface with an average roughness greater than $100 \mathrm{~nm}$. This micro-crystallite morphology can be seen more clearly in the height mode image presented in Figure 5.7. 


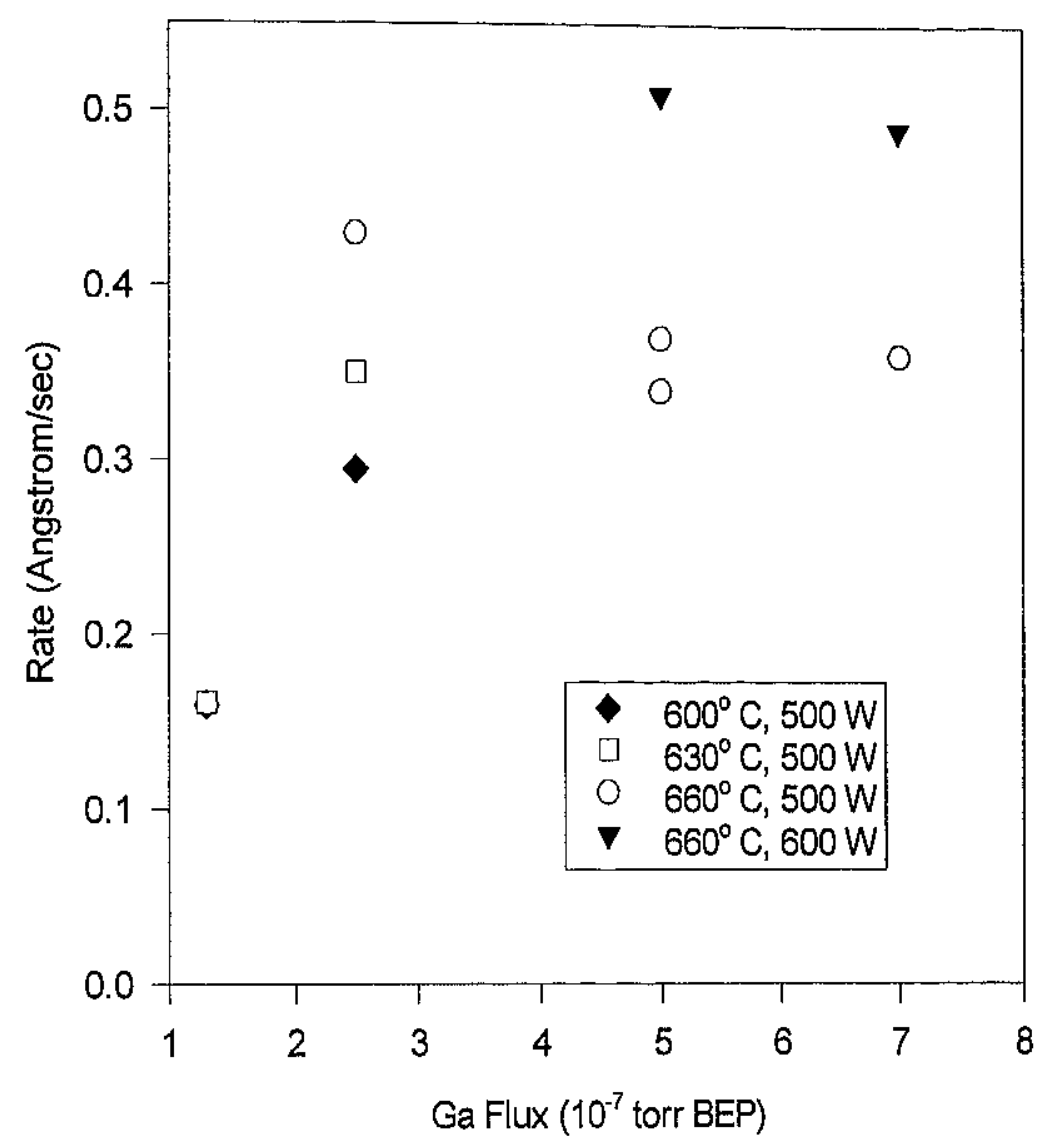

Figure 5.5 Growth rate as a function of Ga flux for several temperatures. Estimated error in growth rate determination is approximately $10 \%$. 

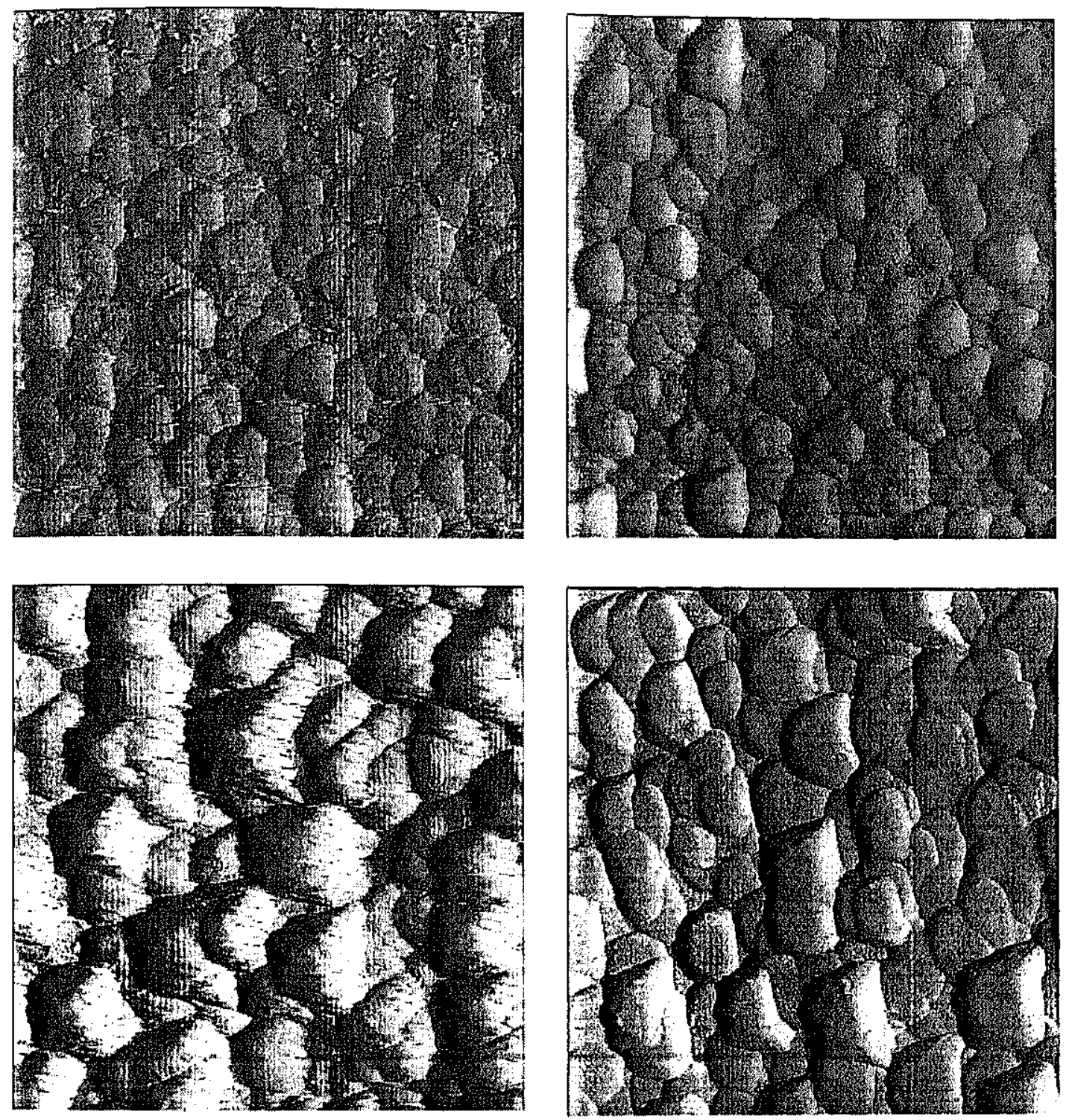

Figure 5.6 (Clockwise from top left) AFM micrographs of samples grown under $2.5 \mathrm{x}$ $10^{-7}$ torr $\mathrm{Ga}$ at substrate temperatures of $550,580,600,630^{\circ} \mathrm{C}$ and $4 \mathrm{SCCM}$ nitrogen at 500 watts. Measured rms surface roughness was $>100 \mathrm{~nm}$ in all cases. 


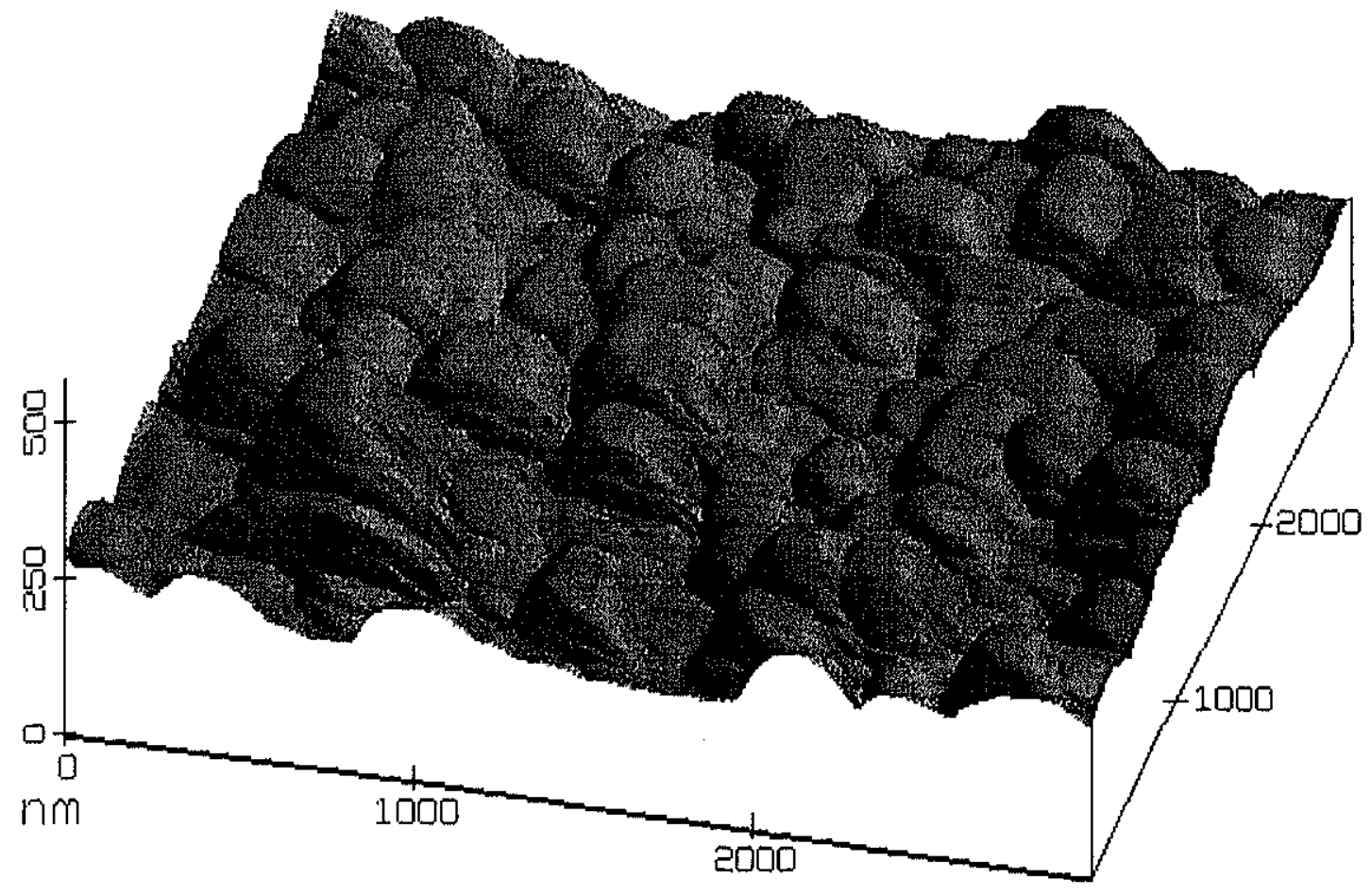

Figure 5.7 Height mode image of one of the samples shown in figure 5.6 
Increasing the substrate temperature to $660^{\circ} \mathrm{C}$ brings about an interesting change. Figure 5.8a shows an AFM micrograph of a film grown at $660^{\circ} \mathrm{C}$ with $2.5 \times 10^{-7} \mathrm{BEP}$ Ga and 4 SCCM N $\mathrm{N}_{2}$ flow at 500 watts. After $3000 \AA$ of growth, the film still exhibits well-defined 3-D growth generating a surface with an average roughness of $150 \mathrm{~nm}$. Xray measurements showed the film to be single-crystal but with a fairly large full-widthat-half-maximum (FWHM) of 400 arc minutes. The film shown in Figure $5.8 \mathrm{~b}$ was grown under double the Ga flux used for the sample in Figure 5.8a. The increase in Ga flux brings the film closer to Ga condensation (about $7.0 \times 10^{-7}$ Torr for this temperature) and produces larger nucleation domains. More importantly, however, is the evident change in growth mode between the two films. The film shown in Figure $5.8 \mathrm{~b}$ displays the beginnings of a distinctly two-dimensional growth. Nucleation domains are showing signs of coalescence and the average surface roughness has been reduced to about $20 \mathrm{~nm}$. The island tops are relatively flat and have well-defined sub-nanometer steps that correspond to one or two monolayers of growth. X-ray measurements on this film showed a reduction in FWHM to about 120 arc minutes. This morphological change was evident in all films grown in the Ga-rich regime with substrate temperatures higher than $660^{\circ} \mathrm{C}$.

Films grown above $660^{\circ} \mathrm{C}$ exhibit signs of the beginnings of 2-D growth. A more standard growth sequence involves an annealing step following a growth interruption. Two samples were grown to investigate the effect of an annealing step on layer morphology. Figure 5.9 shows AFM micrographs of layers grown at $670^{\circ} \mathrm{C}$ under $6 \mathrm{SCCM}$ of $\mathrm{N}_{2}$ at 500 watts and $5.0 \times 10^{-7} \mathrm{BEP}$ Ga. Figure $5.9 \mathrm{a}$ again displays flattopped domains and monolayer terraces. Figure $5.9 \mathrm{~b}$ shows an identical sample except that growth was interrupted after $100 \AA$. The film was then annealed under nitrogen at 670 ${ }^{\circ} \mathrm{C}$ for 20 minutes, and growth was resumed. The resulting film displays almost complete coalescence with a surface roughness of about $1.5 \mathrm{~nm}$. X-ray measurements on this film showed a FWHM of roughly 51 arc minutes. 


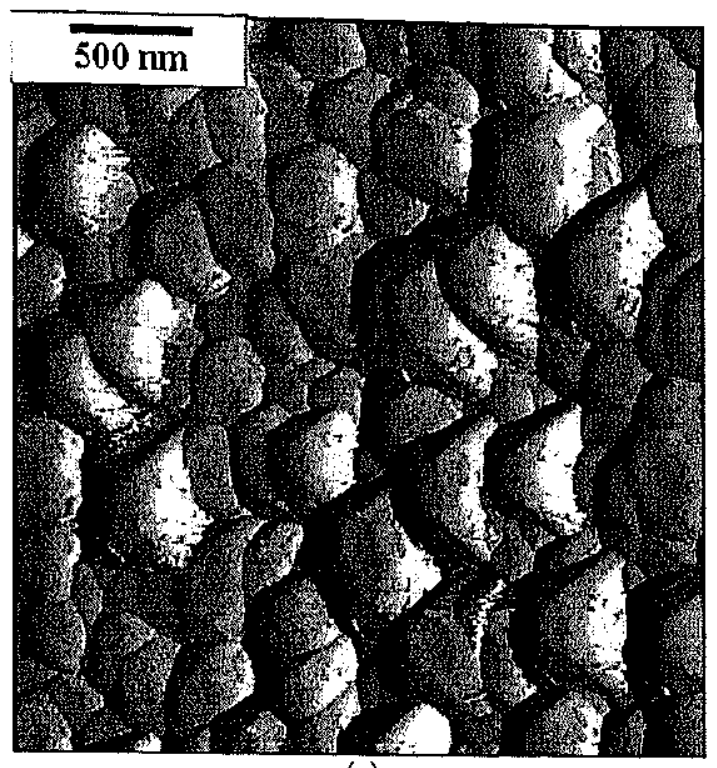

(a)

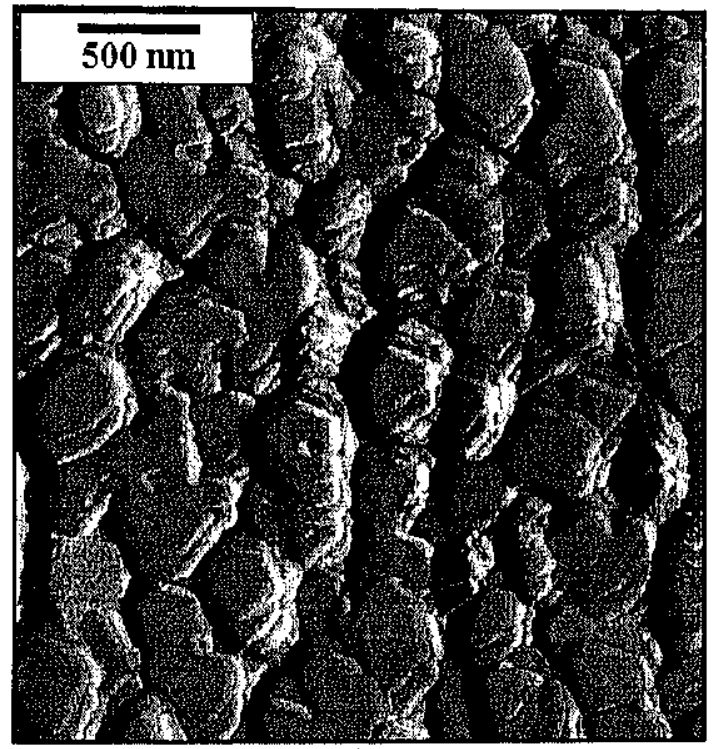

(b)

Figure 5.8 AFM micrographs of samples grown at $660^{\circ} \mathrm{C}$ and $4 \mathrm{SCCM}$ nitrogen at 500 Watts. The top image is from a sample grown under $2.5 \times 10^{-7}$ Torr Ga, while the lower image is from a sample grown under $5.0 \times 10^{-7}$ Torr. 


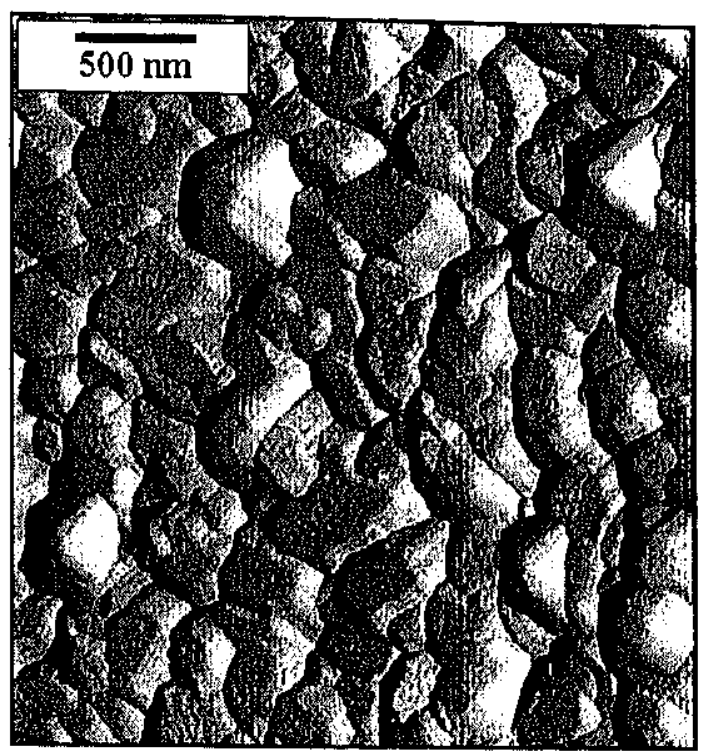

(a)

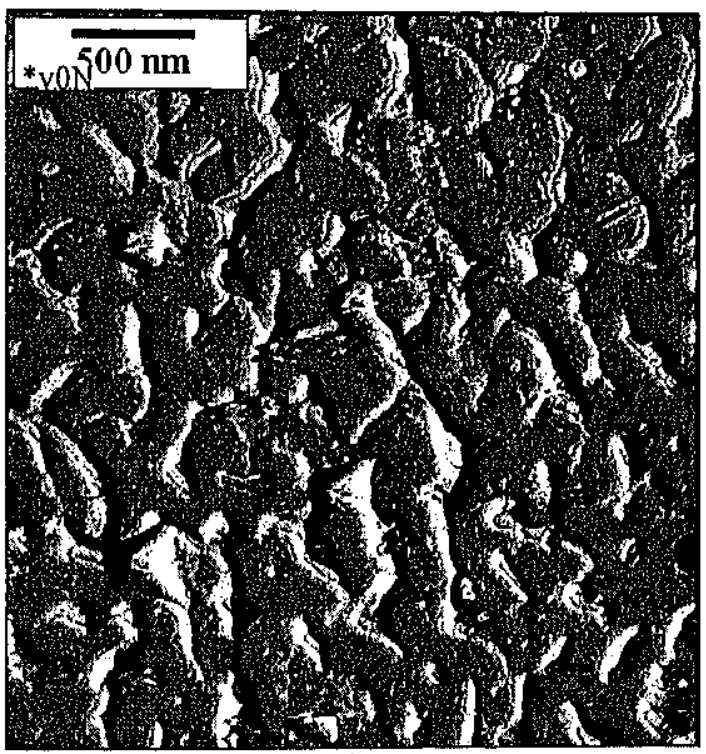

(b)

Figure 5.9 AFM micrographs of samples grown at $670^{\circ} \mathrm{C}$ and $6 \mathrm{SCCM}$ nitrogen at 500 Watts. The top image is from a sample grown under $5.0 \times 10^{-7}$ Torr Ga, while the lower image is from a sample grown under $7.0 \times 10^{-7}$ Torr. 
To briefly summarize then, optimal surface morphologies require growth at substrate temperatures above $660^{\circ} \mathrm{C}$ in a Ga-rich flux (nearly at the point of $\mathrm{Ga}$ condensation). Annealing the resulting films at or above $660^{\circ} \mathrm{C}$ leads to almost complete coalescence of the nucleation domains. By growing films in the Ga-rich regime at substrate temperatures above $660^{\circ} \mathrm{C}$ we have found conditions that produce a mean island size of $\sim 0.3$ micron (as seen in Figure 5.10). This corresponds to a defect density around $1 \times 10^{9} \mathrm{~cm}^{-2}$. Thus, we are poised to begin growth of thicker layers for more complete characterization. 


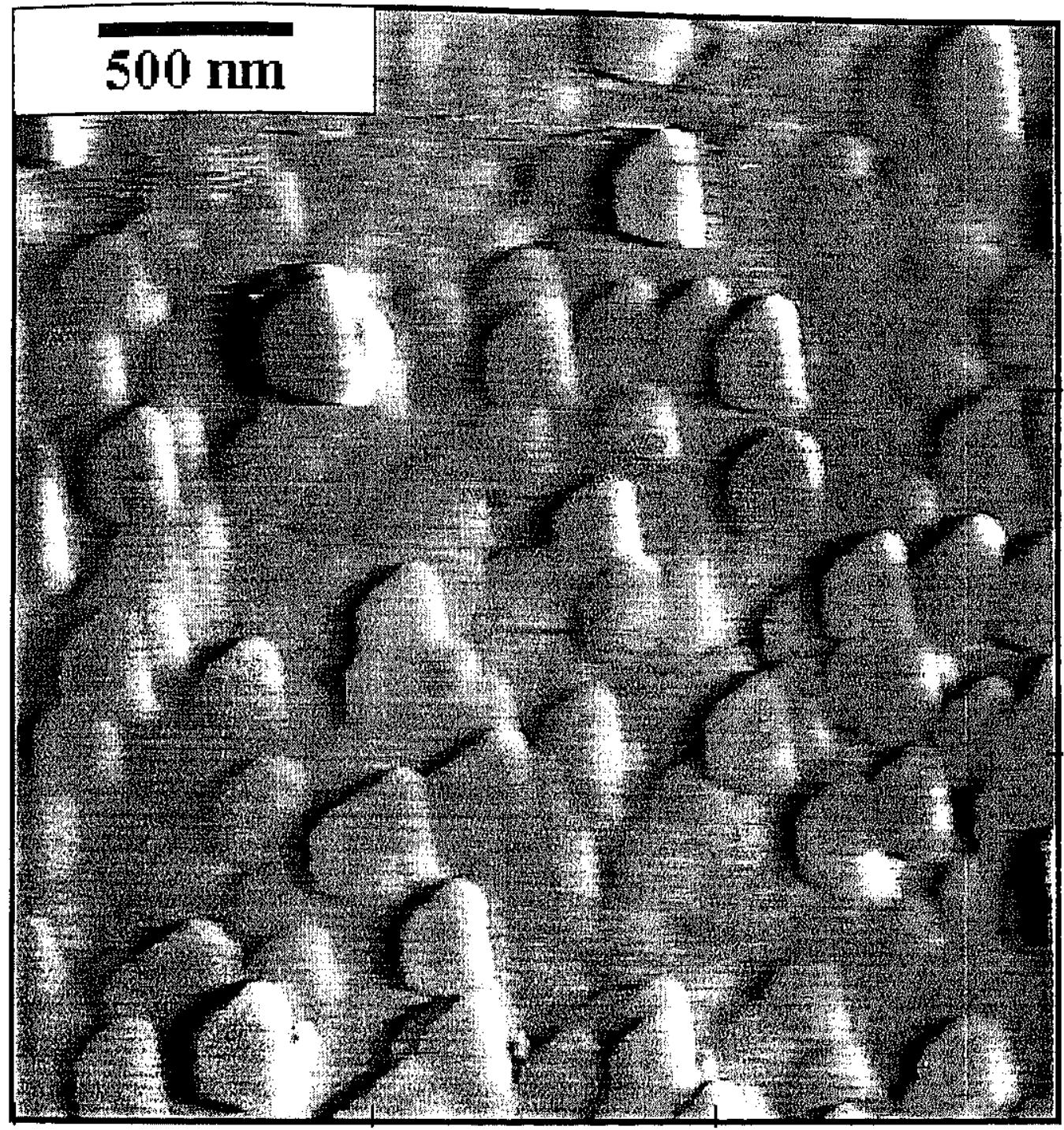

Figure 5.10 Nucleation layer grown at $660^{\circ} \mathrm{C}$ under $5.0 \times 10^{-7}$ torr $\mathrm{Ga}$ and $6 \mathrm{SCCM}$ nitrogen at 500 watts. The mean diameter of the islands in the sample was 0.3 micron. 


\section{Growth of $\mathrm{GaN}$}

With the established conditions for buffer layer growth as described in the previous section, it was possible to grow films for a more detailed characterization of material properties including PL, AFM, and Hall measurements. This next sequence of layer growths would serve as a baseline to guide subsequent studies using $\mathrm{GaN}$. As part of this study, some samples were grown under a flux of hydrogen (atomic and molecular) to ascertain its effects, if any, on the growth. All films were grown on an optimized GaN buffer layer $100-200 \AA$ thick grown under $5.0 \times 10^{-7} \mathrm{Torr} \mathrm{Ga}$ at $670^{\circ} \mathrm{C}$ and $6 \mathrm{SCCM} \mathrm{N} 2$ at 500 Watts. The buffer layers were then annealed under the nitrogen flux for 10 minutes. The sapphire substrates were prepared as described in Chapter 5 except that several samples were mounted to the molybdenum heater block using molten indium and a molybdenum mask instead of graphite.

\subsection{Basic Growth}

A series of layers was grown with thicknesses varying from about 0.5 to 1.1 microns. Figures 6.1a and b are AFM micrographs of two such films grown at $670{ }^{\circ} \mathrm{C}, 6$ SCCM and 500 Watts plasma power. Again, this clearly shows the need for Ga-rich growth as the film grown under $7.0 \times 10^{-7}$ Torr $\mathrm{Ga}(6.1 \mathrm{~b})$ exhibits significantly larger domains than the film grown under $5.0 \times 10^{-7}$ Torr(6.1a). With further increase in substrate temperature, the surface morphology continues to improve. Figure 6.2 shows two films which were grown at $730^{\circ} \mathrm{C}$ under $5.0 \times 10^{-7}$ Torr Ga, 6 SCCM N 2 and 600 Watts. These films show almost complete coalescence of the nucleation islands with the exception of the pyramidal 'hillocks' evident in Figure 6.2a. The flat areas in both films have an average roughness of $\sim 1.5-2.0 \mathrm{~nm}$ and X-ray FWHM of about $4-5$ arc minutes. However, increasing the substrate temperature from $670^{\circ} \mathrm{C}$ to $730^{\circ} \mathrm{C}$ has brought about a 


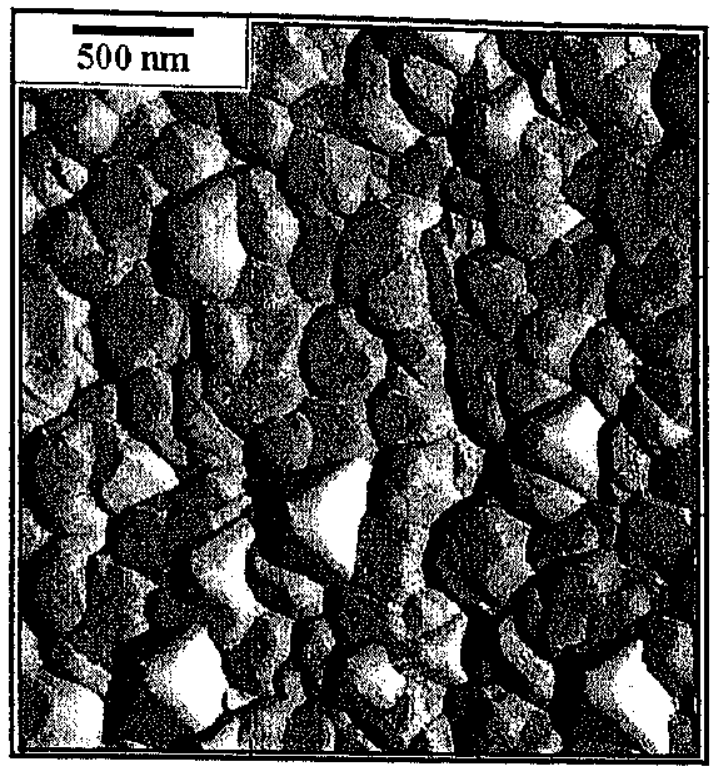

(a)

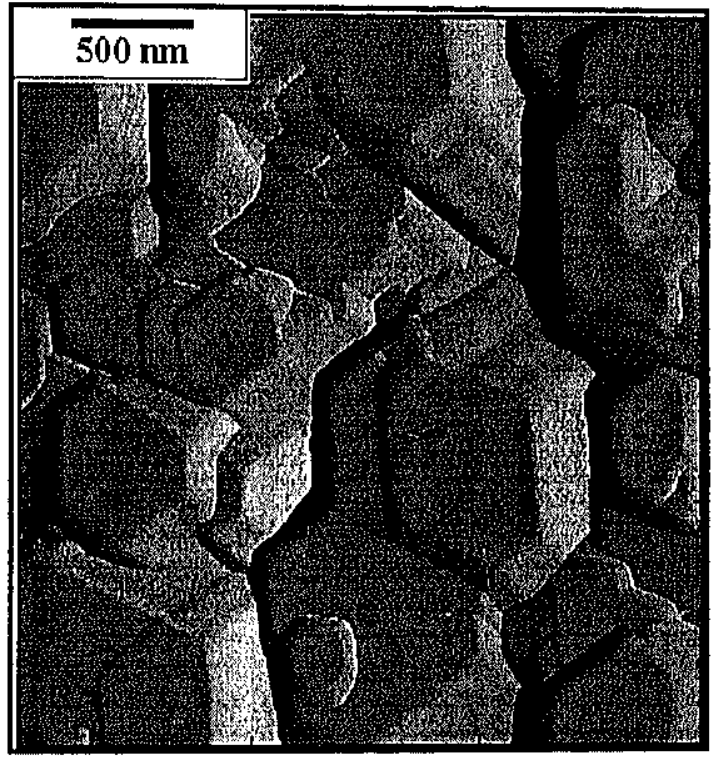

(b)

Figure 6.1 AFM micrographs of samples grown at $670^{\circ} \mathrm{C}$ and $6 \mathrm{SCCM}$ nitrogen at 500 Watts. The top image is from a sample grown under $5.0 \times 10^{-7} \mathrm{Torr} \mathrm{Ga}$, while the lower image is from a sample grown under $7.0 \times 10^{-7} \mathrm{Torr}$. 


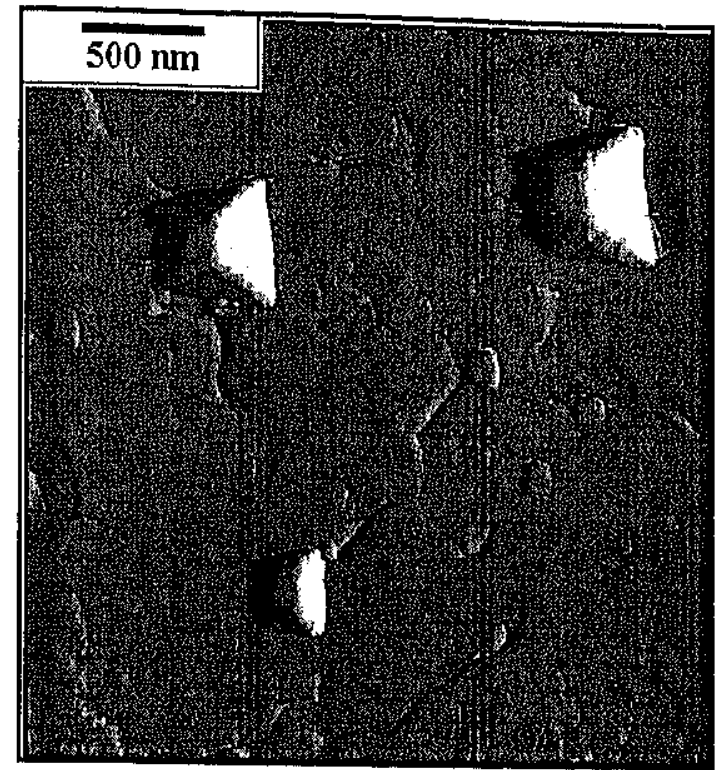

(a)

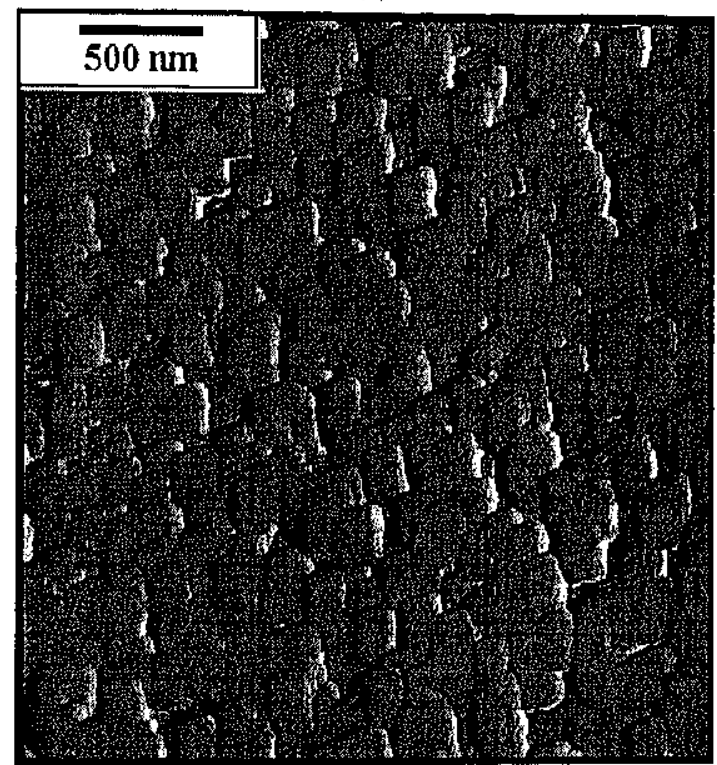

(b)

Figure 6.2 AFM micrographs of samples grown at $730^{\circ} \mathrm{C}$ and $6 \mathrm{SCCM}$ nitrogen at 600 Watts. The top image is from a sample grown under $5.0 \times 10^{-7}$ Torr Ga, while the lower image is from a sample grown under $7.0 \times 10^{-7}$ Torr. 
decrease in the overall growth rate, reducing it by. This may be due, in part, to the increasingly 2-D mode of growth exhibited in this film. It may also be due to increased Ga desorption as prior studies have indicated that the Ga desorption rate increases rapidly above $700^{\circ} \mathrm{C}$.

The sample shown in Figure 6.2a has also been examined by Dr. Linda Romano (Xerox PARC) using transmission electron microscopy (TEM). Figure 6.3 is a crosssectional TEM micrograph of this sample. The dark lines running vertically up from the substrate are the remaining boundaries between nucleation domains. Although most such defects have an edge-type nature, this was not the case in this layer. It appears that sufficient coalescence has removed them, leaving only non-edge type dislocations.

Also, the TEM study identified another type of defect, inversion domain boundaries (IDBs), where the in-plane axes of neighboring domains are effectively reversed relative to one another. A schematic of the IDB and its possible relation to substrate surface inhomogeneities is shown in Figure 6.4.

In sharp contrast to GaN grown in other labs by MBE, the IDBs in our sample are estimated to fill less than $10 \%$ of the sample volume. Typical MBE grown GaN films exhibit defect levels around 50\% [30].

By counting the dislocations which intersect the surface, as seen on Figure 6.3, it is possible to estimate the dislocation density. For this film, the density works out to roughly $2 \times 10^{9} \mathrm{~cm}^{-2}$ which compares favorably with the best reported by any other growth technique (MOCVD: $\sim 7 \times 10^{8} \mathrm{~cm}^{-2}$ ). This also compares quite well with the density of $1 \times 10^{9} \mathrm{~cm}^{-2}$ estimated from nucleation island size and resultant structure observed in AFM from our AFM study on layers grown with similar nucleation conditions. Note, however, that even though this defect density is comparable to the lowest reported, it is still five orders-of-magnitude higher than would be tolerable in other semiconductor systems, like GaAs. 


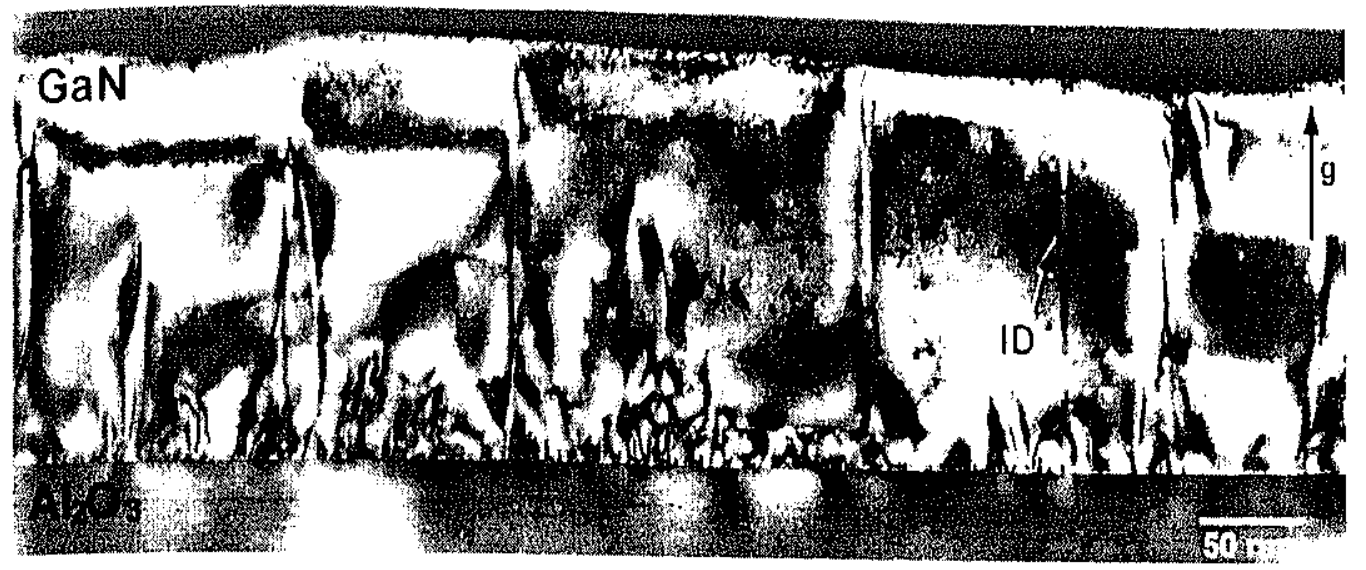

Figure 6.3 Dark field TEM image of sample 9571 (AFM image shown in figure 6.2a). 'ID' denotes an Inversion Domain. The remaining defects are non-edge type dislocations. (Photo courtesy of Dr. Linda Romano, Xerox PARC) 


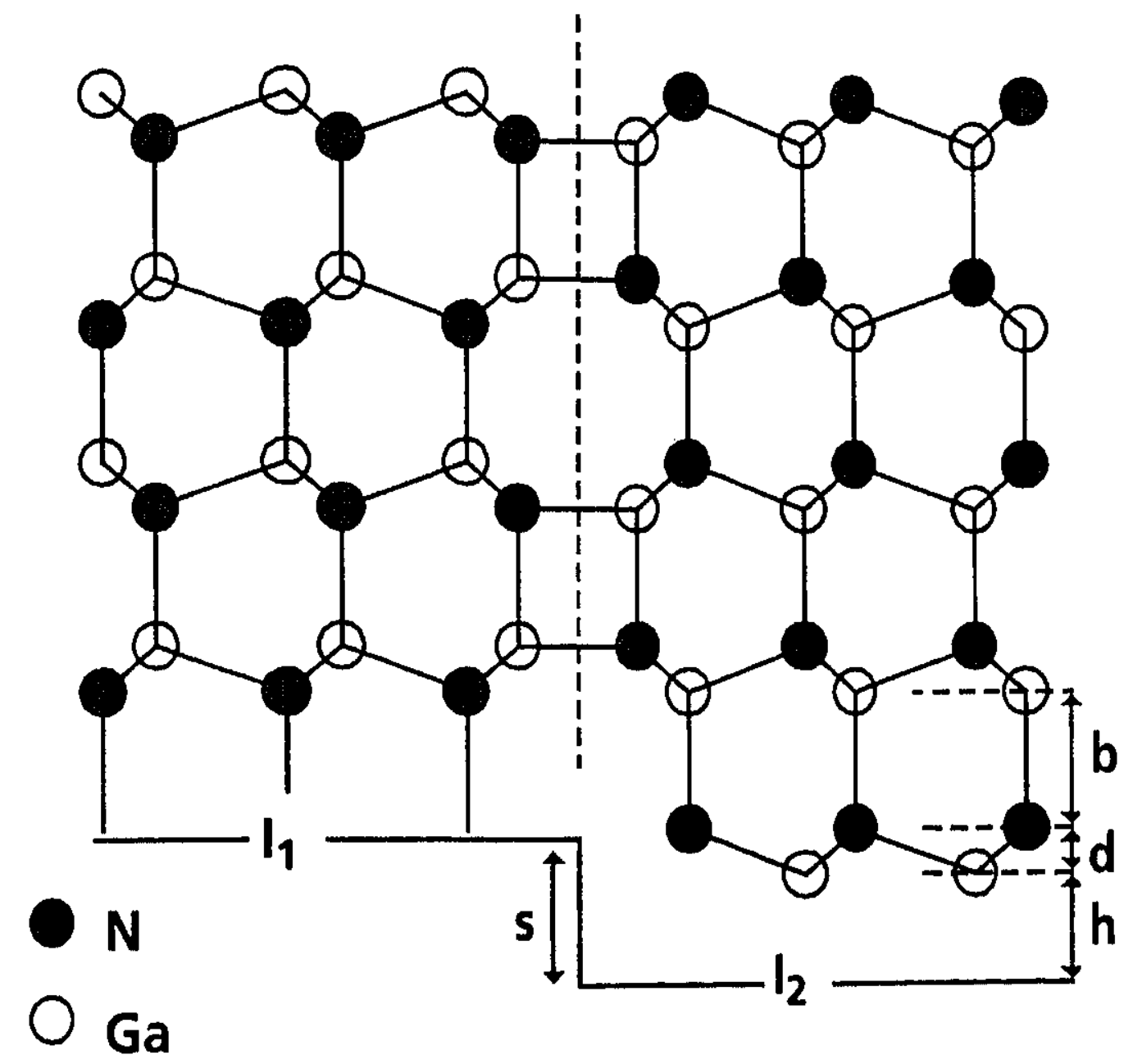

Figure 6.4 Schematic of Inversion Domain structure. ('S' represents a substrate step or defect which may be responsible for creating this type of defect.) (Drawing courtesy of Dr. Linda Romano, Xerox, PARC) 
Photoluminescence was performed at liquid helium temperature by Prof. Giles in her laboratory on several samples prepared in this growth run. The samples exhibited band-edge emission at about $365 \mathrm{~nm}(3.47 \mathrm{eV})$. This is the so-called $\mathrm{I}_{1}$ peak, and is commonly ascribed to an exciton bound to a nitrogen vacancy. However, this assignment has not been clearly proven. The $\mathrm{I}_{1}$ peak is, however, typically observed for material grown under Ga-rich (nitrogen deficient) conditions. All samples grown at or below 660 ${ }^{\circ} \mathrm{C}$ displayed an additional broad luminescence band centered at about $560 \mathrm{~nm}(2.2 \mathrm{eV})$, as seen in Figure 6.5a. While the origin of this yellow luminescence is still controversial [31], it is commonly attributed to deep states in the bandgap involving impurities or native defects [32]. There is a growing belief in the community that this luminescence is associated with edge-type dislocations occurring at coalescence boundaries. Interestingly, the layers grown at $730^{\circ} \mathrm{C}$ did not exhibit a detectable level of this yellow luminescence, as seen in Figure $6.5 \mathrm{~b}$. This may indicate that temperatures above $660^{\circ} \mathrm{C}$ are necessary for high quality material. In low temperature PL measurements, the layers grown at $730{ }^{\circ} \mathrm{C}$ exhibited primarily near-bandedge luminescence related to excitonic transition, again suggesting an improvement in at least those aspects of layer quality probed by PL.

The yellow luminescence seen in the PL data can also be seen, at room temperature, with the aid of an optical microscope with an UV light source. Samples exhibiting the yellow PL band could be seen to shine bright yellow under UV excitation. With the aid of an optical microscope, it was possible to perform UV fluorescence microscopy (UVFL) to examine the spatial distribution of the yellow emission. Figure 6.6 shows one such micrograph. One can clearly see individual microcrystallites resolvable as $0.5-0.3$ micron in diameter. It is apparent that the luminescence is not uniform across the sample and varies from one microcrystallite to another. Some isolated microcrystallites exhibited blue luminescence. Occasional samples exhibited weak PL centered near $400 \mathrm{~nm}$ or $440 \mathrm{~nm}$ when measured using the 

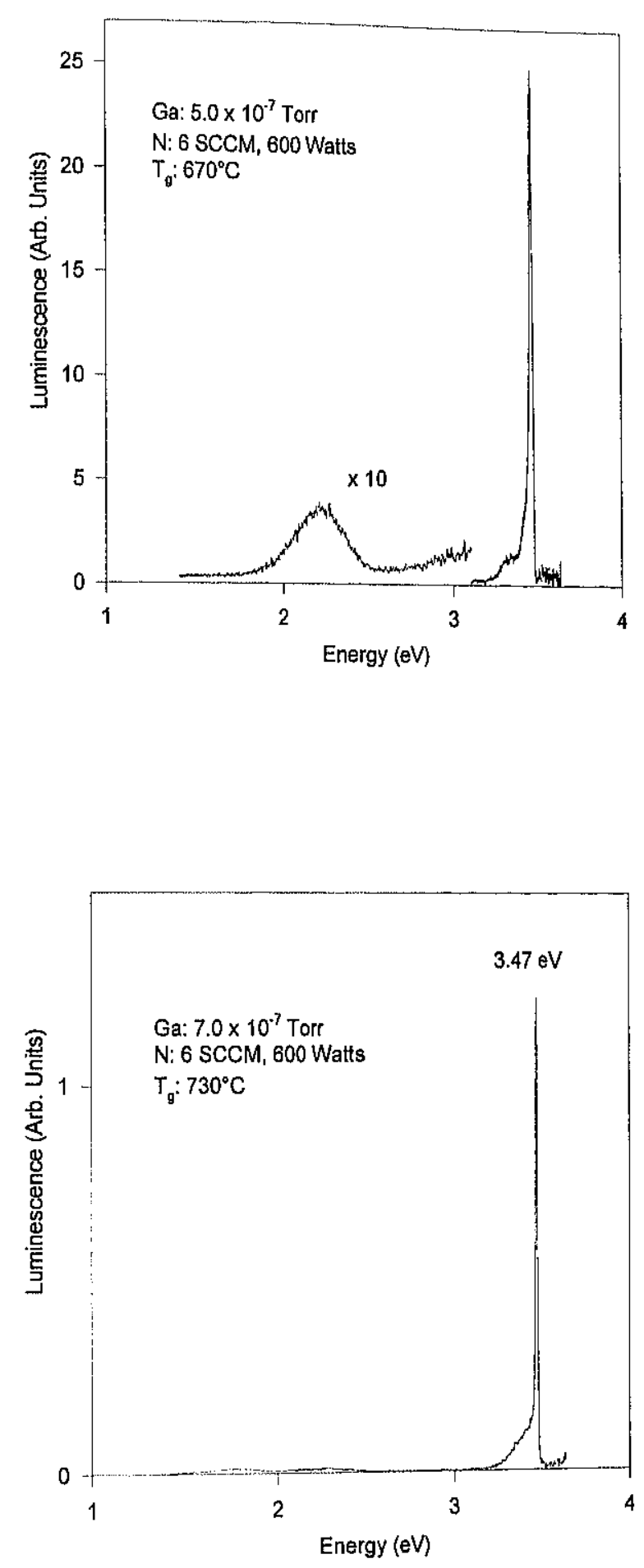

Figure 6.5 Photoluminescence spectra for two samples grown at $660^{\circ} \mathrm{C}$ (top) and $730^{\circ} \mathrm{C}$ (bottom). Note suppression of the $2.2 \mathrm{eV}$ 'yellow' luminescence in the higher temperature growth. 


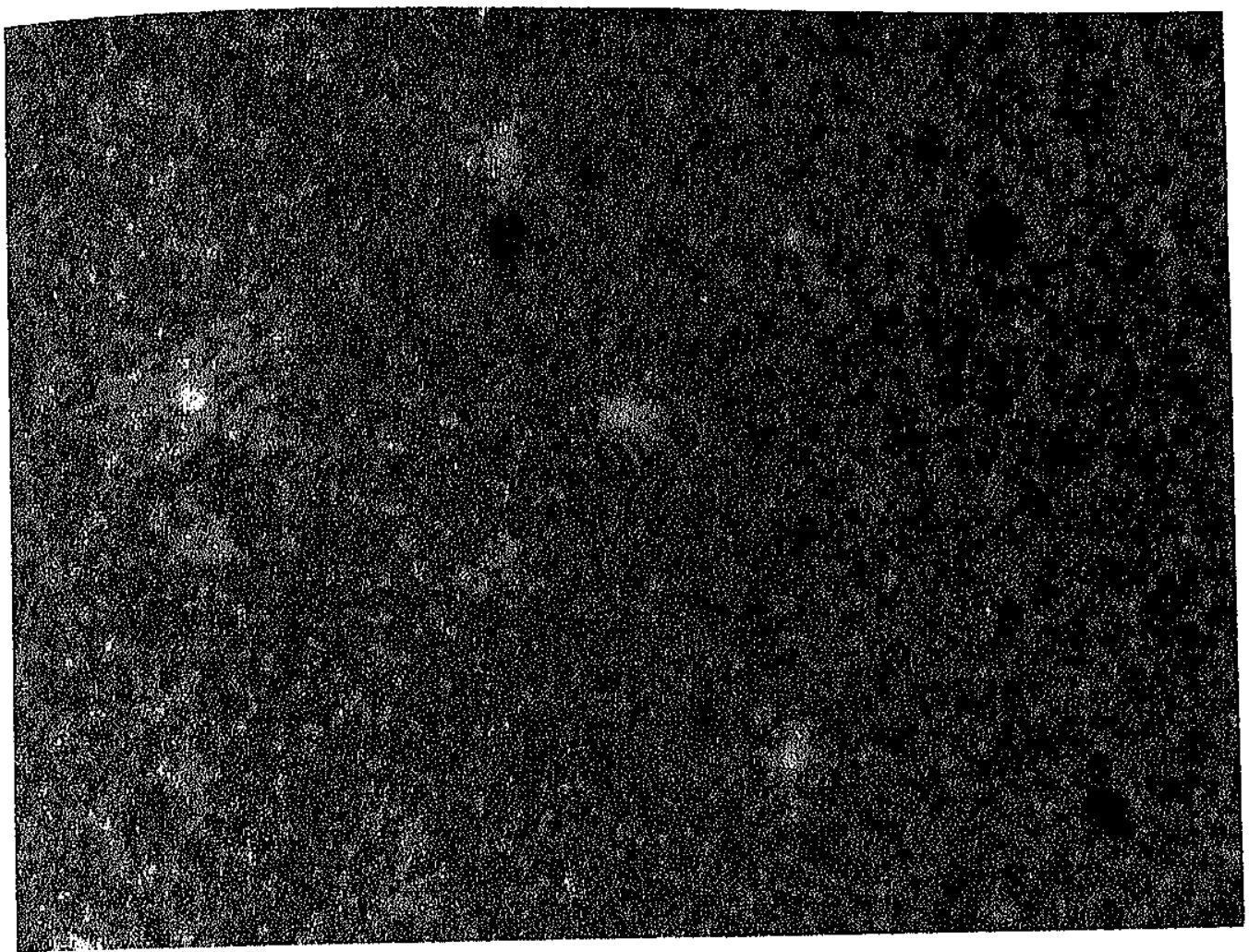

Figure 6.6 Ultraviolet Fluorescence micrograph of 'yellow luminescence' seen in PL spectra. 
standard PL setup. The UVFL result indicates that this may actually originate from localized impurities or defects. The samples grown at $730{ }^{\circ} \mathrm{C}$ did not exhibit any detectable yellow fluorescence at room temperature.

Hall measurements were performed on these samples at room temperature, A clear trend emerged in these measurements. For samples grown at $730^{\circ} \mathrm{C}$, we observed the high n-type carrier concentration $\left(6.3 \times 10^{17}-2.5 \times 10^{18} \mathrm{~cm}^{-3}\right)$ reported for most samples grown by any technique. Mobilities ranged from $4.9 \mathrm{~cm}^{2} / \mathrm{Vs}$ to $469.2 \mathrm{~cm}^{2} / \mathrm{Vs}$, with the lowest values coming predominantly from the higher temperature growths. Of interest is one measurement indicating a mobility of $4,000 \mathrm{~cm}^{2} / \mathrm{Vs}$ at low carrier concentration. This, of course, needs to be carefully re-evaluated and a temperature dependent study performed to determine compensation ratios.

This n-type conduction is often attributed to nitrogen vacancies, as it is believed that most higher temperature growths take place under conditions conducive to vacancy formation [33]. An alternative theory is that the n-type carrier concentration is related to defects resulting from the decomposition of the $\mathrm{GaN}$ during typical growth conditions [34]. This would occur where the growth rate starts decreasing with temperature with the attendant increase in Ga desorption rate. Rubin, et al. [35], observed a dramatic decrease in n-type carrier concentration upon going to lower growth temperatures, with mobilities as high as $1200 \mathrm{~cm}^{2} / \mathrm{Vs}$ for a carrier concentration of $10^{14} \mathrm{~cm}^{-3}$. In addition, they observed as-grown p-type conduction, possibly related to a reduction of the n-type background due to this unknown defect.

We also observed a dramatic decrease in n-type carrier concentration upon going to the low temperature growths. For samples grown at $660^{\circ} \mathrm{C}$ or less, carrier concentrations were both $\mathrm{n}$ - and p-type, ranging from $9 \times 10^{12}$ to $4 \times 10^{15} \mathrm{~cm}^{-3}$. The mobilities covered a wide range, probably indicating various degrees of compensation and possibly mixed conduction in some cases. 
A future study may be to use higher temperatures to obtain structural perfection, followed by lower temperature growth to eliminate (or minimize) this n-type defect.

\subsection{Effects of Hydrogen on the Growth of GaN}

It is well known that hydrogen easily bonds to the surface of other semiconductor systems, such as silicon or germanium [36]. There is also evidence that atomic hydrogen alters the growth kinetics in GaAs [37,38]. For several samples, hydrogen was incorporated into the growth flux during $\mathrm{GaN}$ growth. $\mathrm{H}_{2}$ passed through the thermal cracker described earlier produced a low flux of atomic hydrogen. The unfilled symbols in Figure 6.7 show the change in growth associated with the introduction of hydrogen. As much as a factor of two increase in growth rate was seen for all samples grown under Garich conditions with hydrogen, when compared to their hydrogen-free counterparts. This is perhaps the most intriguing finding of this study. Based on this, it is likely that hydrogen is playing an important role in the growth kinetics of MOCVD, MOMBE, and in GSMBE using ammonia. A detailed study of this phenomenon is currently underway in Prof. Myers' research group, funded by the Office of Naval Research from a proposal based on the research described in this thesis.

Hydrogen incorporating directly into the film cannot account for the increase on growth rate. Figure 6.8 shows two films grown under identical conditions except that one was grown with an additional $1 \times 10^{-6}$ Torr hydrogen in the vacuum chamber. It is readily seen that the film grown with hydrogen has a much smaller domain size than the hydrogen-free film. It was shown earlier that both a decrease in domain size and an increase in growth rate, in the Ga-rich regime, can be explained by a shift to a more nitrogen sufficient growth. This implies that the presence of hydrogen effects the growth kinetics, effectively increasing the active nitrogen flux. It is possible that the hydrogen bonds loosely to the growing surface where it attracts incident nitrogen atoms. The 


\section{Growth Rate vs. Ga Flux}

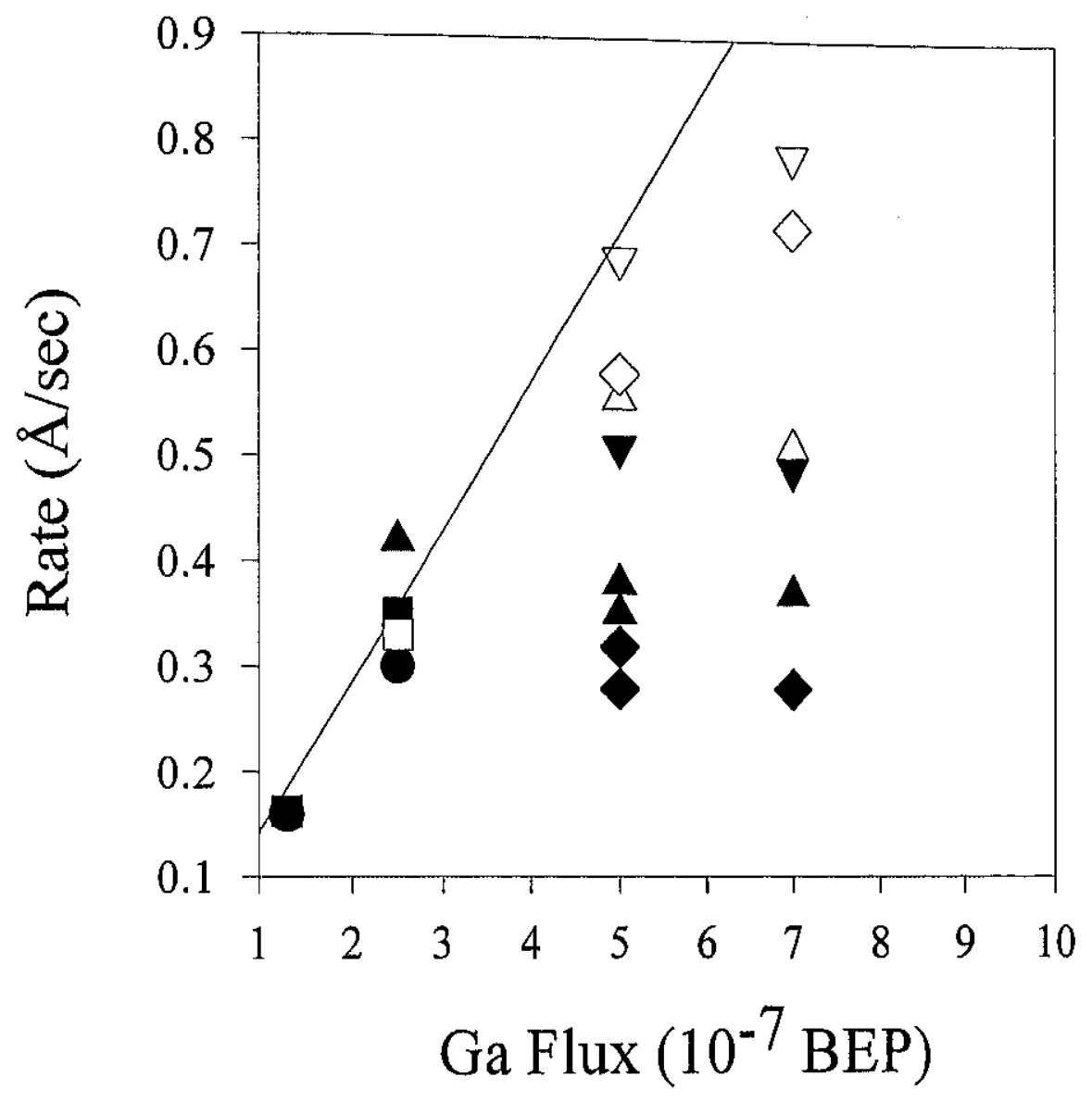

Figure 6.7 Graph of growth rate versus Ga flux for various growth conditions.

Unfilled symbols correspond to growth conditions of similar filled symbols but with the addition of atomic hydrogen to the growth flux. 

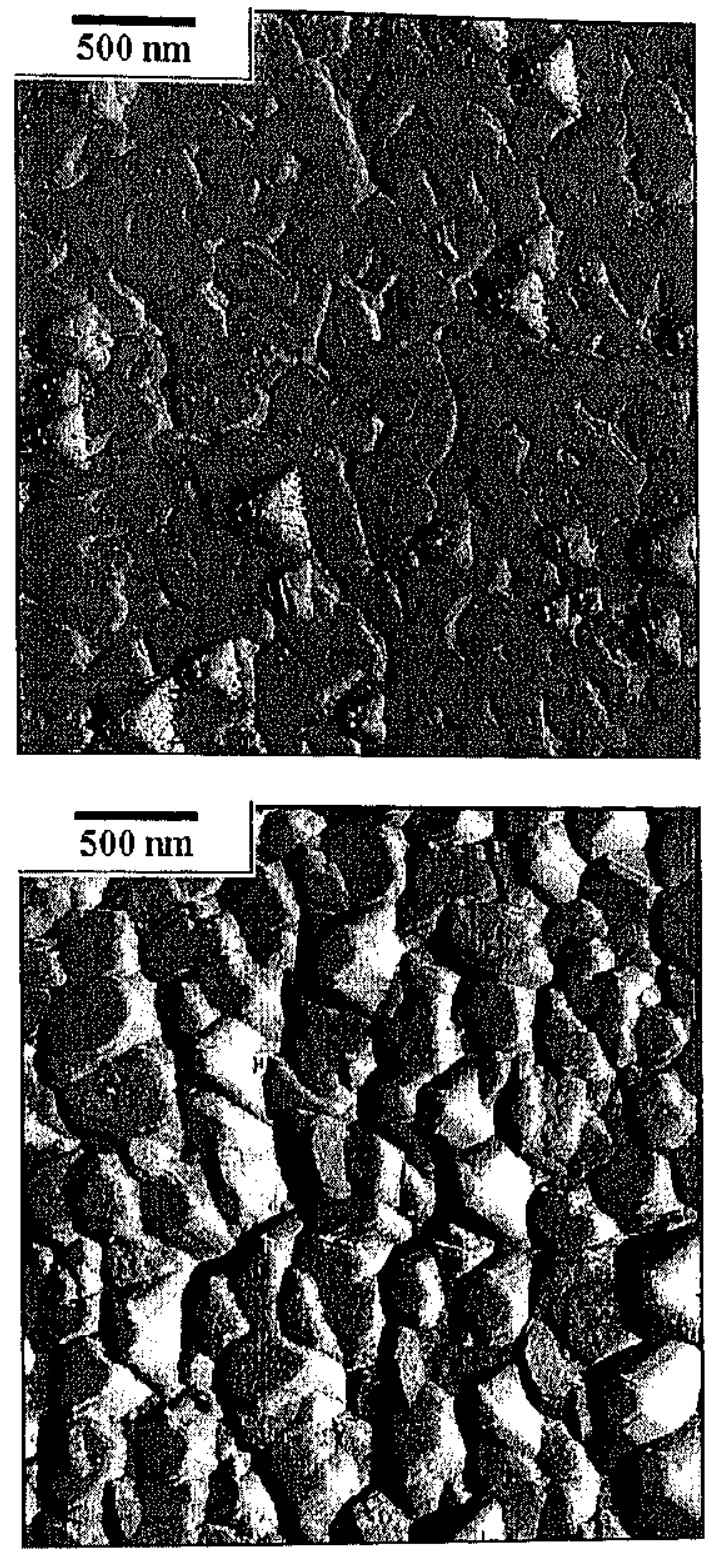

Figure 6.8 AFM micrographs of two samples grown at $670^{\circ} \mathrm{C}$ under $5.0 \times 10^{-7}$ torr $\mathrm{Ga}$ and $6 \mathrm{SCCM}$ nitrogen at 500 watts. Top image is from a sample grown without hydrogen in the growth flux. Bottom image is from a sample grown with $1.0 \times 10^{-6}$ torr added to growth flux through thermal cracker. Note smaller domain size similar to increasing nitrogen flux. 
nitrogen atoms attach to this hydrogen layer thereby increasing the nitrogen residence time. This allows adsorbed $\mathrm{Ga}$ atoms more time to diffuse to within an interaction distance to bond with the nitrogen. Doubling the hydrogen flux to $2.0 \times 10^{-6}$ Torr resulted in no noticeable difference in growth rate, implying that the reaction does not depend on the incident flux of hydrogen, just on its presence.

Structural and electrical properties were indistinguishable between films grown with and without hydrogen. The best GaN films grown with hydrogen exhibited x-ray diffraction FWHM between 4 and 5 arc minutes. Hall measurements suggested a possible factor of two improvement in carrier concentration, and an order-of-magnitude improvement in mobility, between similar samples.

Figure 6.9 shows PL results for two films grown with hydrogen. Again the low temperature growth exhibited a yellow luminescence band while the high temperature growths exhibited mainly band-edge luminescence. Figure $6.9 \mathrm{~b}$ shows the $3.47 \mathrm{eV}$ bound-exciton peak and the absence of PL related to deep levels, indicating that growth under hydrogen does not degrade layer quality. 

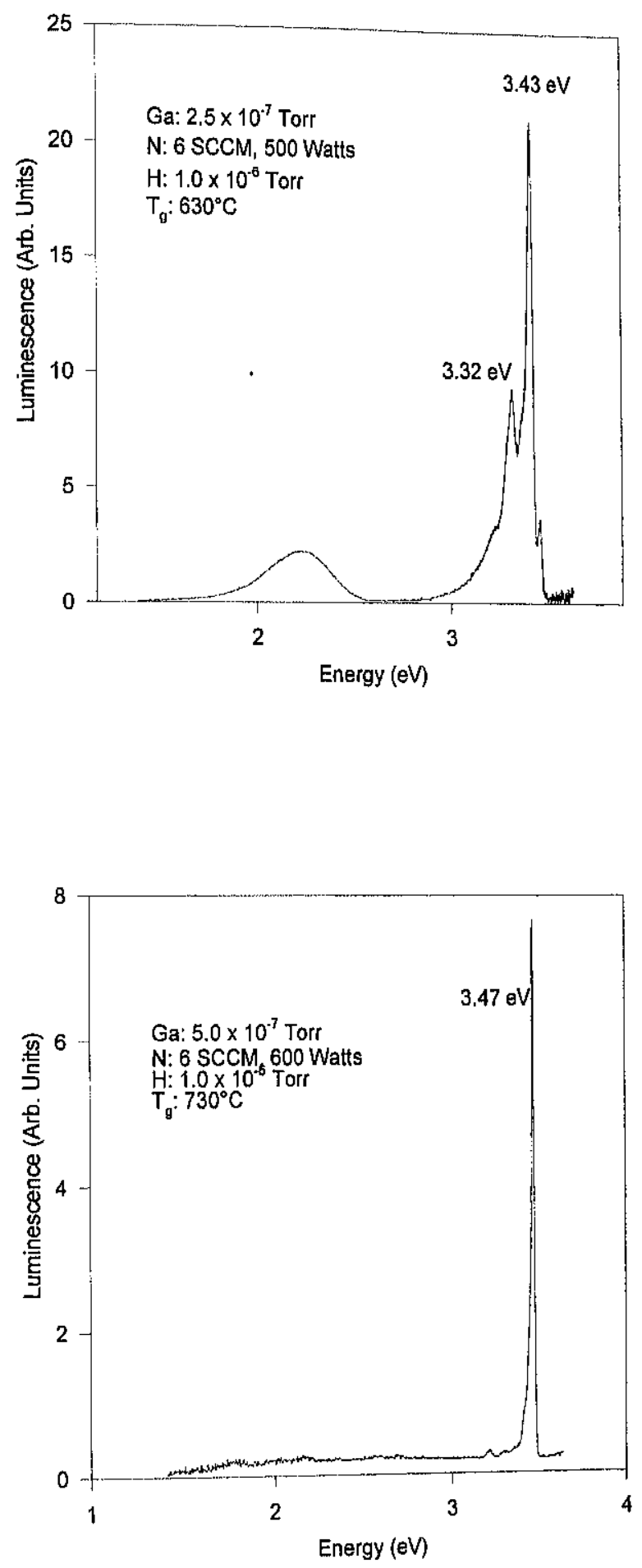

Figure 6.9 PL spectra for two samples grown with atomic hydrogen in the growth flux. Spectra are virtually unchanged compared to similar non-hydrogen growths. 


\section{Summary and Future Areas for Research}

I GaN is an interesting, challenging and technologically! important semiconductor system 'Its potential applications range from acid-resistant coatings to optoelectronic devices operating in the blue and near-UV wavelengths) (For this reason, considerable effort has been, and is being, expended by this lab, and by others/around the world, toward growing high quality material and understanding its properties, In recent years, numerous advances in the growth and processing of GaN have sparked new interest in its potential. Newer growth techniques have improved the crystallinity and helped demonstrate p-type material growth (The use of buffer layers for growth on sapphire substrates has significantly improved layer quality, both electrically and mechanically. The upcoming availability of better lattice-matched substrates and, possibly, GaN substrates for homoepitaxial growth are sure to bring about even greater improvements.

The four major goals of my research were achieved. Characterization of the RF radical source was conducted using optical emission and mass spectroscopies. It was shown that the output flux consists of atomic and molecular nitrogen, and ions of each in varying concentrations. For a given source operating power, the percent of the flux converted to atomic nitrogen is shown to decrease with increasing gas flow into the source. When converted to actual fluence, however, the atomic nitrogen flux increases with increasing flow and power. Ion fluxes were shown to be low (less than $0.03 \%$ of the total output) for this source when using an aperture plate with small $(<0.5 \mathrm{~mm}$ diameter) exit holes. Larger exit holes exhibit higher fluxes of high energy ions. This is likely due to a decrease in the recombination of ions in the boundary layer of the exit holes.

As a part of this research effort, thirty-two GaN films have been grown to date in the WVU Molecular Beam Epitaxy lab. The primary goal of these growths was to study the initial nucleation of $\mathrm{GaN}$ on sapphire. Nucleation layers were grown at substrate 
temperatures ranging from $550^{\circ} \mathrm{C}$ to $670^{\circ} \mathrm{C}$, under $\mathrm{Ga}$ fluxes ranging from $1.3-5.0 \times 10^{-7}$ Torr. Active nitrogen was supplied by a RF nitrogen source. Examination of layer surface morphologies by atomic force microscopy showed significant improvement in layer quality when growths were conducted at higher temperatures under a Ga-rich growth flux. In fact, the highest layer quality occurred at Ga fluxes just short of where Ga begins to condense out onto the sample surface. Growths under these conditions displayed the beginnings of a change in growth mode from 3-D micro-crystallites to a flatter, more 2-D growth. These growth conditions were also shown to promote larger nucleation islands, which in turn lowers the final density of domain-edge related crystal defects. Conditions were reported which resulted in $2 \times 10^{9} \mathrm{~cm}^{-2}$ defect densities, comparable to the $7 \times 10^{8} \mathrm{~cm}^{-2}$ commonly considered to be state-of-the-art. Annealing of the nucleation layers was shown to promote a high degree of domain coalescence, resulting in films with average surface roughness less than $5 \mathrm{~nm}$.

Thicker layers $(<1 \mu \mathrm{m})$ were grown for some of the higher temperature, higher quality conditions. Characterization by photoluminescence showed that growth at lower temperatures led to the phenomenon of "yellow luminescence", commonly attributed to poor structural quality. This luminescence was greatly suppressed in layers grown above $670^{\circ} \mathrm{C}$. TEM measurements confirmed the low density of large-scale crystalline defects and demonstrated that those remaining were primarily non-edge type dislocations, giving hope of further reductions in density with two dimensional growth.

Several films were grown with atomic hydrogen added to the growth flux. This resulted in growth rate enhancements of as much as a factor of two. The hydrogen is most likely altering the growth kinetics, increasing the residence time of nitrogen on the sample surface. Inclusion of hydrogen was shown to have no discernible effect on material properties under the conditions utilized here.

Work is already in progress to further the study reported here. Much interest has been expressed in the effects of hydrogen on growth kinetics. Further work needs to be 
done with growths under a wider range of hydrogen fluxes to determine if there is a limit to the possible benefits of this technique. Growth techniques need to be further refined to promote the early onset of 2-D growth and further reduce the level of intrinsic crystal defects. Further material characterization also needs to be conducted to determine what growth conditions are necessary, not only for high structural quality but high electrical and optical quality as well. In addition, recent SIMS studies of two samples indicated a potentially disturbing fact; at least those samples, and quite likely most of the others, are heavily contaminated with boron (see figure 7.1). The contamination most likely stems from the breakdown of the pyrolitic boron nitride crucible in the nitrogen source. A new crucible has been installed and future growths will hopefully determine the effect of the presence of boron. In the end, it may prove that boron inclusion is necessary for high quality layer growth.

Thus, the research presented in this thesis represents a solid baseline for future efforts in understanding the growth and properties of GaN. 


\section{GNSA-9567}

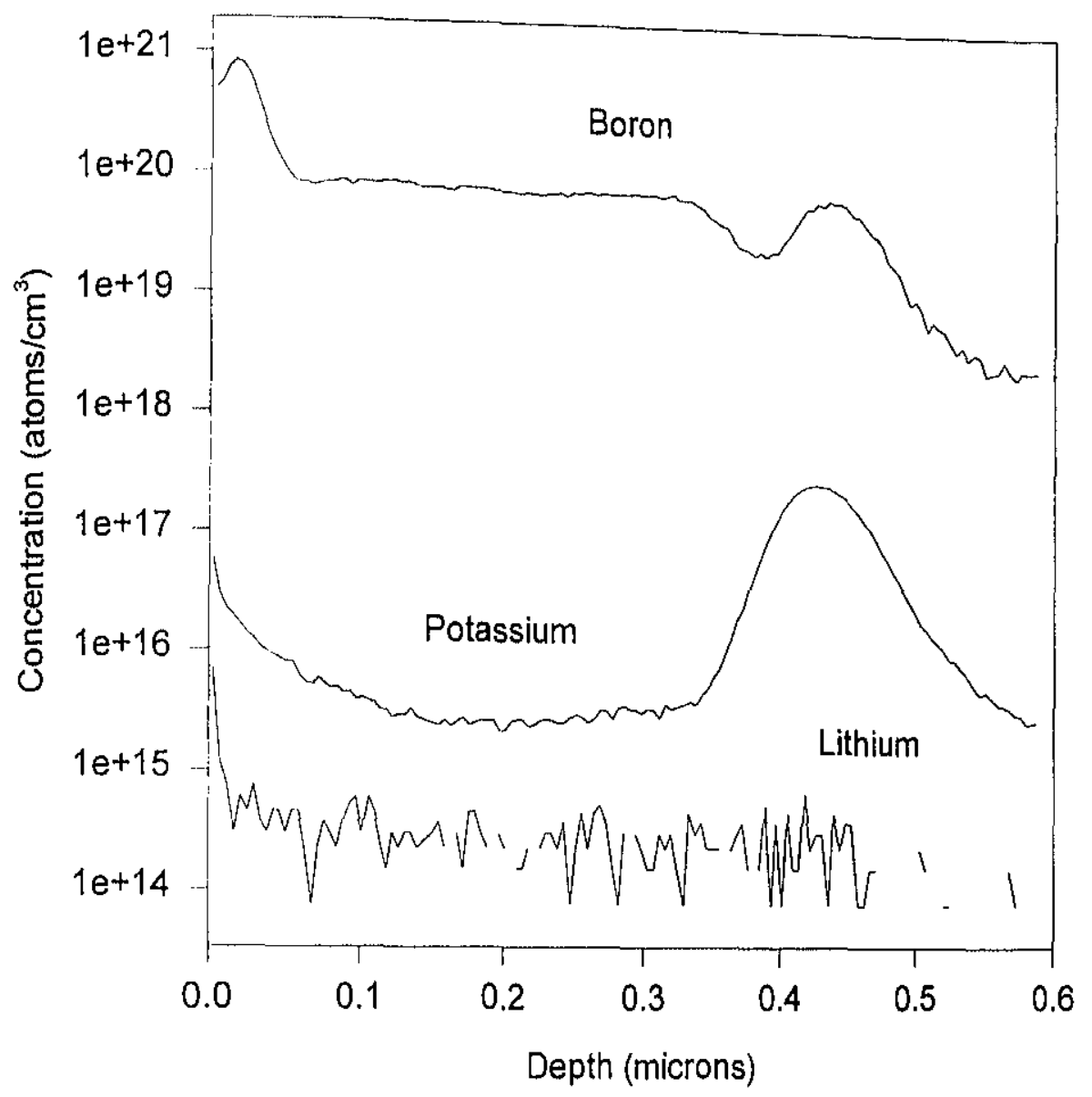

Figure 7.1 SIMS spectrum for sample 9567 indicating a very high level of boron inclusion. Similar results were obtained for sample 9569. This may indicate boron inclusion in all samples grown to date. 


\section{References}

[1] H. Morkoç, S. Strite, G.B. Gao, M.E. Lin, B. Sverdlov and M. Burns, J. Appl.

Phys. 76, 1363 (1994).

[2] Z. Yu, PhD Dissertation, Physics Dept., WVU (1996).

[3] See for example: Mat. Res. Soc. Proc. Vol. 283 (1993).

[4] W. Götz, N.M. Johnson, J. Walker, D.P. Bour, H. Amano and I. Akasaki, Appl. Phys. Lett. 67, 2666 (1995).

[5] M.S. Brandt, N.M. Johnson, R.J. Molnar, R. Singh and T.D. Moustakas, Appl. Phys. Lett 64, 2264 (1994).

[6] H. Amano, M. Kito, K. Hiramatsu and J. Akasaki, Jpn. J. Appl. Phys. 28, L2112 (1989).

[7] S. Nakamura, T. Mukai, M. Senoh and N. Iwasa, Jpn. J. Appl. Phys. 31, L139 (1992).

[8] S. Nakanura, T. Mukai and M. Senoh, Jpn. J. Appl. Phys. 30, L1998 (1991).

[9] R.C. Powell, N.E. Lee, Y.W. Kim and J.E. Greene, J. Appl. Phys. 73, 189 (1993).

[10] R.J. Molnar and T.D. Moustakas, J. Appl. Phys, 76, 4587 (1994).

[11] Z. Yang, L. Li and W. Wang, Appl. Phys. Lett. 67, 1686 (1995).

[12] M. Asif Khan, T.D. Olson, J.N. Kuznia, W.E. Carlos and J.A. Freitas, Jr. 74, 5901 (1993).

[13] S. Nakamura, T. Mukai and M. Senoh, J. Appl. Phys. 71, 5543 (1992).

[14] S. Strite and H. Morkoc, J. Vac. Sci. Technol. B 10, 1237 (1992). 
[15] J.S. Hwang, A.V. Kuznetsov, S.S. Lee, H.S. Kim, J.G. Choi and P.J. Chong Journal of Crystal Growth 142, 5 (1994).

[16] J.N. Kuznia, M. Asif Khan and D.T. Olson, J. Appl. Phys. 73, 4700 (1993).

[17] A.Y. Cho and J.R. Arthur, Progress in Solid-State Chemistry 10, 157 (1975).

[18] J.A. Venables, G.D.T. Spiller and M. Hanbücken, Rep. Prog. Phys. 47, 399 (1984).

[19] B.A. Joyce, Rep. Prog. Phys. 37, 363 (1974).

[20] L.J. Van der Pauw, Philips Res. Rep. 13, 1 (1958).

[21] G. Binnig, C.F. Quate and Ch. Gerber, Phys. Rev. Lett. 56, 930 (1986).

[22] D. Rugar and P. Hansma, Physics Today, 23 (October 1990).

[23] See also: S. Kumar, MS Thesis, Dept. of Chem. Eng., 1996.

[24] R.P. Vaudo, J.W. Cook, Jr. and J.F. Schetzina, J. Crystal Growth 138, 430 (1994).

[25] W.E. Hoke, P.J. Lemonias and D.G. Weir, J. Crystal Growth 111, 1024 (1991).

[26] R.P. Vaudo, J.W. Cook, Jr. and J.F. Schetzina, J. Vac. Sci. Technol B 12, 1232 (1994).

[27] R.P. Vaudo, Z. Yu, J.W. Cook, Jr. and J.F. Schetzina, Optics Letters 18, 1843 (1993).

[28] J.M. Van Hove, G.J. Cosimini, E. Nelson, A.M. Wowchak and P.P. Chow, Journal of Crystal Growth 150, 908 (1995).

[29] T.D. Moustakas, T. Lei and R.J. Molnar, Physica B, 185, 36 (1993). 
[30] L.T. Romano, J.E. Northrup and M.A. O'Keefe, Appl. Phys. Lett. 69, 2394 (1996).

[31] P. Kung, X. Zhang, D. Walker, A. Saxler, J. Piotrowski, A. Rogalski and M. Razeghi, Appl. Phys. Lett. 67, 3792 (1995).

[32] R. Singh, R.J. Molnar, M.S. Ünlü and T.D. Moustakas, Appl. Phys. Lett. 64, 336 (1994).

[33] J. Neugebauer and C.G. Van de Walle, Phys. Rev. B 50, 8067 (1994).

[34] N. Newman, J. Ross and M. Rubin, Appl. Phys. Lett. 62, 1242 (1993).

[35] M. Rubin, N. Newman, J.S. Chan, T.C. Fu and J.T. Ross, Appl. Phys. Lett. 64, 64 (1994).

[36] J.A. Schaefer, Physica B 170, 45 (1991).

[37] Y.J. Chun, Y. Okada and M. Kawabe, Jpn. J. Appl. Phys. 32, L1085 (1993). 


\begin{tabular}{|c|c|c|c|c|c|c|c|c|c|c|c|c|c|c|c|c|}
\hline $\begin{array}{l}\text { Sample } \\
\text { Number }\end{array}$ & $\begin{array}{l}\text { Growth } \\
\text { Temp. }\end{array}$ & $\begin{array}{l}\text { Pga } \\
\text { (10e-7 } \\
\text { Torr) }\end{array}$ & $\begin{array}{l}\mathrm{N} \\
\text { (SCCM) }\end{array}$ & $\begin{array}{l}\text { Power } \\
\text { (Watts) }\end{array}$ & $\begin{array}{l}\text { Sub. } \\
\text { etch }\end{array}$ & $H(e-6)$ & $\begin{array}{l}N \\
\text { PR }\end{array}$ & $m_{t}^{m n}$ & $\begin{array}{l}\text { rate } \\
(\AA / s)\end{array}$ & $\begin{array}{l}\text { Gro } \\
\text { (hrs) }\end{array}$ & $\begin{array}{l}\text { Thick } \\
(\mu \mathrm{m})\end{array}$ & $\begin{array}{l}\text { Buf } \\
(A)\end{array}$ & $\begin{array}{l}\mathrm{BPga} \\
(\mathrm{e}-7)\end{array}$ & $\begin{array}{l}\text { BN2 } \\
\text { (SCCM) }\end{array}$ & $\begin{array}{l}\text { Aneal } \\
\text { (min) }\end{array}$ & BTm \\
\hline GNSA-9544 & 630 & 2.5 & 4 & 500 & no & no & no & Gra & 0.37 & 6 & 0.8 & no & & & & \\
\hline GNSA-9545 & 580 & 2.5 & 4 & 500 & yes & no & no & Gra & 0.32 & 6 & 0.7 & no & & & & \\
\hline GNSA-9546 & 600 & 2.5 & 4 & 500 & yes & no & no & Gra & 0.30 & 6 & 0.65 & no & & & & \\
\hline GNSA-9547 & 550 & 2.5 & 4 & 500 & yes & no & no & Gra & 0.30 & 6 & 0.65 & no & & & & \\
\hline GNSA-9548 & 630 & 2.5 & 4 & 500 & yes & no & no & Gra & 0.35 & 6 & 0.75 & no & & & & \\
\hline GNSA-9549 & 600 & 5 & 4 & 500 & yes & no & no & Gra & 0.00 & 6 & & no & & & & \\
\hline GNSA-9550 & 630 & 1.3 & 4 & 500 & yes & no & no & Gra & 0.00 & 6 & & no & & & & \\
\hline GNSA-9551 & 630 & 5 & 4 & 500 & yes & no & no & Gra & 0.00 & 6 & & no & & & & \\
\hline GNSA-9552 & 600 & 1.3 & 4 & 500 & yes & no & no & Gra & 0.16 & 6 & 0.35 & no & & & & \\
\hline GNSA-9553 & 660 & 5 & 4 & 500 & yes & no & no & Gra & 0.37 & 3 & 0.4 & no & & & & \\
\hline GNSA-9554 & 660 & 2.5 & 4 & 500 & yes & no & no & Gra & 0.35 & 6 & 0.75 & no & & & & \\
\hline GNSA-9555 & 630 & 2.5 & 4 & 500 & yes & no & yes & Gra & 0.32 & 3 & 0.35 & no & & & & \\
\hline GNSA-9556 & 630 & 2.5 & 4 & 500 & yes & yes & no & Gra & 0.56 & 3 & 0.6 & no & & & & \\
\hline GNSA-9557 & 630 & 5 & 6 & 500 & yes & no & no & Gra & 0.00 & 3 & & no & & & & \\
\hline GNSA-9558 & 630 & 2.5 & 4 & 500 & yes & no & no & Gra & 0.00 & 3 & & 50 & 2.5 & & & 450 \\
\hline GNSA-9559 & 660 & 5 & 6 & 500 & yes & no & no & Gra & 0.32 & 3 & 0.35 & no & & & & \\
\hline GNSA-9560 & 660 & 5 & 6 & 500 & yes & no & no & Gra & 0.00 & 0.05 & & & & & & \\
\hline GNSA-9561 & 670 & 5 & 6 & 500 & yes & no & no & Gra & 0.27 & 3 & 0.29 & 100 & 5 & & & \\
\hline GNSA-9562 & 670 & 5 & 6 & 500 & yes & 1 & no & Gra & 0.56 & 3 & 0.6 & 100 & 5 & & & \\
\hline GNSA-9563 & 670 & 7 & 6 & 500 & yes & no & no & Gra & 0.37 & 3 & 0.4 & 100 & 5 & & & \\
\hline GNSA-9564 & 670 & 7 & 6 & 500 & yes & 1 & no & Gra & $0 . \overline{51}$ & 3 & 0.55 & 200 & 7 & & & \\
\hline GNSA-9565 & 670 & 7 & 6 & 500 & yes & 2 & no & Gra & 0.56 & 3 & 0.6 & 200 & 5 & & & \\
\hline GNSA-9566 & 670 & 2.5 & 6 & 0 & yes & 2 & no & Gra & 0.02 & 6 & 0.045 & 200 & 5 & 6 & 10 & 670 \\
\hline GNSA-9567 & 670 & 5 & 6 & 600 & yes & no & no & Gra & 0.51 & 3 & 0.55 & 200 & 5 & 6 & 10 & 670 \\
\hline GNSA-9568 & 670 & 7 & 6 & 600 & yes & 1 & no & Gra & 0.79 & 3 & 0.85 & 200 & 5 & 6 & 10 & 670 \\
\hline GNSA-9569 & 670 & 5 & 6 & 600 & yes & 1 & no & Gra & 0.69 & 3 & 0.75 & 200 & 5 & 6 & 10 & 670 \\
\hline GNSA-9570 & 670 & 5 & 6 & 600 & yes & $1-\mathrm{H}_{2}$ & no & Gra & 0.32 & 3 & 0.35 & 200 & 5 & 6 & 10 & 670 \\
\hline GNSA-9571 & 730 & 5 & 6 & 600 & yes & no & no & In & $0 . \overline{32}$ & 6 & 0.7 & 200 & 5 & 6 & 10 & 670 \\
\hline
\end{tabular}




\begin{tabular}{|c|c|c|c|c|c|c|c|c|c|c|c|c|c|c|c|c|}
\hline $\begin{array}{l}\text { Sample } \\
\text { Number }\end{array}$ & \begin{tabular}{|l|} 
Growth \\
Temp.
\end{tabular} & $\begin{array}{l}\text { Pga } \\
\text { (10e-7 } \\
\text { Torr) } \\
\end{array}$ & $\begin{array}{l}N \\
\text { (SCCM) }\end{array}$ & \begin{tabular}{|l|} 
Power \\
(Watts)
\end{tabular} & $\begin{array}{l}\text { Sub. } \\
\text { etch }\end{array}$ & $H(e-6)$ & $\begin{array}{l}\mathrm{N} \\
\mathrm{PR}\end{array}$ & $\begin{array}{l}m n \\
t\end{array}$ & $\begin{array}{l}\text { rate } \\
(\AA / s)\end{array}$ & \begin{tabular}{|l|} 
Gro \\
(hrs)
\end{tabular} & \begin{tabular}{|l|} 
Thick \\
$(\mu \mathrm{m})$
\end{tabular} & $\begin{array}{l}\text { Buf } \\
(\AA)\end{array}$ & $\begin{array}{l}\text { BPga } \\
(e-7)\end{array}$ & \begin{tabular}{|l|} 
BN2 \\
(SCCM)
\end{tabular} & $\begin{array}{l}\text { Aneal } \\
\text { (min) }\end{array}$ & BTm \\
\hline GNSA-9572 & 730 & 7 & 6 & 600 & yes & no & no & In & 0.28 & 6 & 0.6 & 200 & 5 & 6 & 10 & 670 \\
\hline GNSA-9573 & 730 & 5 & 6 & 600 & yes & 1 & no & In & 0.56 & 6 & 1.2 & 200 & 5 & 6 & 10 & 670 \\
\hline GNSA-9574 & 730 & 5 & 6 & 600 & yes & no & no & In & 0.25 & 12 & 1.1 & 200 & 5 & 6 & 10 & 670 \\
\hline GNSA-9575 & 730 & 5 & 6 & 600 & $y$ & no & & & 0.28 & 6 & 0.6 & 200 & 5 & 6 & 10 & 670 \\
\hline GNSA-9611 & 730 & 7 & 6 & 600 & $y$ & 1 & no & $\ln$ & 0.00 & 6 & & 200 & 5 & 6 & 10 & \\
\hline GNSA-9664 & 730 & 5 & 6 & 600 & yes & no & no & $\ln$ & 0.56 & 7 & 1.4 & 200 & 5 & 6 & 10 & 730 \\
\hline GNSA-9665 & 730 & 5 & 6 & 600 & yes & no & no & In & 0.44 & 6 & 0.95 & 200 & 5 & 6 & 10 & 730 \\
\hline GNSA-9666 & 730 & 5 & 6 & 600 & yes & no & no & $\ln$ & 0.00 & 6 & & 200 & 5 & 6 & 10 & 730 \\
\hline GNSA-9667 & 730 & 5 & 6 & 600 & yes & no & no & In & 0.61 & 3 & 0.66 & 200 & 5 & 6 & 10 & 730 \\
\hline GNSA-9668 & 730 & 7 & 6 & 600 & yes & no & no & In & 0.39 & 3 & 0.42 & 200 & 7 & 6 & 10 & 730 \\
\hline GNSA-9669 & 730 & 9 & 6 & 600 & yes & no & no & In & 0.37 & 3 & 0.4 & 200 & 9 & 6 & 10 & 730 \\
\hline GNSA-9670 & 730 & 9 & 6 & 600 & yes & 1 & no & In & 1.11 & 3 & 1.2 & 200 & 5 & 6 & 10 & 730 \\
\hline
\end{tabular}




\begin{tabular}{|l|r|r|l|}
\hline $\begin{array}{l}\text { Sample } \\
\text { Number }\end{array}$ & Carrier Concentration & Mobility & Notes \\
\hline GNSA-9544 & & & \\
\hline GNSA-9545 & & & \\
\hline GNSA-9546 & $-4.30 E+15$ & 280.7 & \\
\hline GNSA-9547 & & & \\
\hline GNSA-9548 & & & \\
\hline GNSA-9549 & & & \\
\hline GNSA-9550 & & & \\
\hline GNSA-9551 & & & \\
\hline GNSA-9552 & & & \\
\hline GNSA-9553 & & & \\
\hline GNSA-9554 & & & \\
\hline GNSA-9555 & & & \\
\hline GNSA-9556 & & & \\
\hline GNSA-9557 & & 46.4 & \\
\hline GNSA-9558 & & 31.8 & \\
\hline GNSA-9559 & & & \\
\hline GNSA-9560 & $-2.10 E+15$ & & \\
\hline GNSA-9561 & & & Same anneal \\
\hline GNSA-9562 & & & same anneal \\
\hline GNSA-9563 & & & \\
\hline GNSA-9564 & & & \\
\hline GNSA-9565 & $-1.90 E+15$ & 10.1 & Buffer layer annealed 20 minutes under N2 \\
\hline GNSA-9566 & & 469.2 & Annealed 10 minutes \\
\hline GNSA-9567 & $2.30 E+15$ & 52.4 & \\
\hline GNSA-9568 & & & \\
\hline GNSA-9569 & $3.80 E+14$ & 328 & \\
\hline GNSA-9570 & $-3.60 E+13$ & 45.9 & \\
\hline GNSA-9571 & & 40.5 & H2 only, no thermal source \\
\hline
\end{tabular}




\begin{tabular}{|l|r|r|l|}
\hline $\begin{array}{l}\text { Sample } \\
\text { Number }\end{array}$ & Carrier Concentration & Mobility & Notes \\
\hline GNSA-9572 & $-2.50 E+18$ & 4.9 & \\
\hline GNSA-9573 & $-1.40 E+18$ & 118 & \\
\hline GNSA-9574 & & & \\
\hline GNSA-9575 & $-6.30 E+17$ & 14.1 & \\
\hline GNSA-9611 & & & Ga flux dropped for 1/2 hr (1036-988degree) \\
\hline GNSA-9664 & & & First hr @805 on dial, remainder @760 \\
\hline GNSA-9665 & & & Tsub fluctuates during growth, N source leaks \\
\hline GNSA-9666 & & & Source leaks @10e-5 torr-1/sec \\
\hline GNSA-9667 & & & \\
\hline GNSA-9668 & & & \\
\hline GNSA-9669 & & & \\
\hline GNSA-9670 & & & \\
\hline
\end{tabular}




\section{APPROVAL OF THE EXAMINING COMMITTEE}
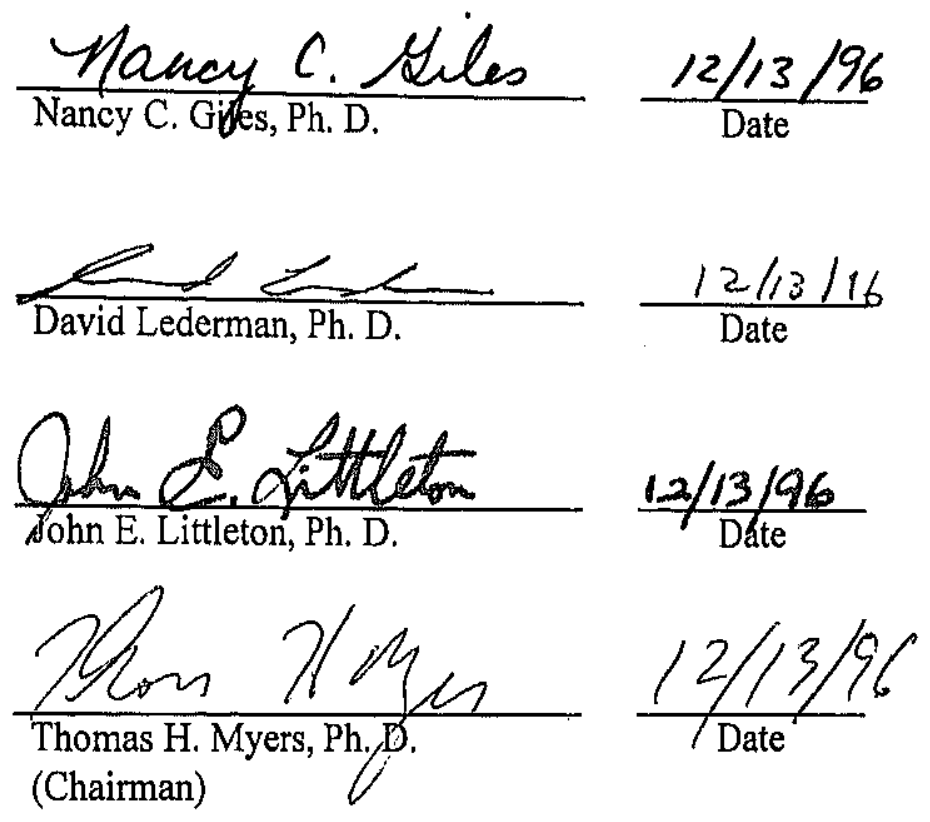